\title{
ATTITUDE ESTIMATION FOR A GRAVITY GRADIENT MOMENTUM BIASED NANOSATELLITE
}

\author{
A Thesis \\ presented to \\ the Faculty of California Polytechnic State University, \\ San Luis Obispo
}

\author{
In Partial Fulfillment \\ of the Requirements for the Degree \\ Master of Science in Aerospace Engineering
}

by

Arash Mehrparvar

November, 2013 
(C) 2013

Arash Mehrparvar

ALL RIGHTS RESERVED

Page ii 
TITLE:

AUTHOR:

DATE SUBMITTED:

COMMITTEE CHAIR:

COMMITTEE MEMBER:

COMMITTEE MEMBER:

COMMITTEE MEMBER:
Attitude Estimation for a Gravity Gradient Momentum Biased Nanosatellite

\author{
Arash Mehrparvar
}

November, 2013

Dr. Jordi Puig-Suari, Professor Aerospace Engineering Department

Dr. Kira Abercromby, Professor Aerospace Engineering Department

Dr. Eric Mehiel, Department Chair Aerospace Engineering Department

Justin Foley, Staff

CubeSat Program 


\begin{abstract}
Attitude Estimation for a Gravity Gradient Momentum Biased Nanosatellite Arash Mehrparvar
\end{abstract}

Attitude determination and estimation algorithms are developed and implemented in simulation for the Exocube satellite currently under development by PolySat at Cal Poly. A mission requirement of $\pm 5^{\circ}$ of attitude knowledge has been flowed down from the NASA Goddard developed payload, and this requirement is to be met with a basic sensor suite and the appropriate algorithms. The algorithms selected in this work are TRIAD and an Extended Kalman Filter, both of which are placed in a simulation structure along with models for orbit propagation, spacecraft kinematics and dynamics, and sensor and reference vector models. Errors inherent from sensors, orbit position knowledge, and reference vector generation are modeled as well. Simulations are then run for anticipated dynamic states of Exocube while varying parameters for the spacecraft, attitude algorithms, and level of error. The nominal case shows steady state convergence to within $1^{\circ}$ of attitude knowledge, with sensor errors set to $3.5^{\circ}$ and reference vector errors set to $2^{\circ}$. The algorithms employed have their functionality confirmed with the use of STK, and the simulations have been structured to be used as tools to help evaluate attitude knowledge capabilities for the Exocube mission and future PolySat missions.

Keywords: attitude, estimation, Extended Kalman filter, TRIAD, spacecraft dynamics, gravity gradient, momentum wheel, spherical damper, cubesat, nanosatellite 


\section{ACKNOWLEDGMENTS}

I'd like to thank my parents, who have always told me I'm not stupid and pushed me hard because of it. I would not have been at this stage today if it wasn't for them so the whole "you'll thank me later" thing is very true. I'd also like to thank my brother since he's awesome, and oddly enough it's his birthday today.

Thanks to Dr. P for being my advisor and putting up with me. Even though he likes to leave the country a lot, if you do get to sit him down he's very helpful. Almost everything he's suggested I add to this thesis has been good stuff.

Special thanks to Dr. Abercromby for being the best lady ever and always setting aside time to go over things when it seemed like no other faculty cared much.

Thanks to the Wolf for putting up with my constant inquiries about everything, although a lot of times I was sorry I ever asked.

Huge thanks to the CubeSat program here at Poly for funding me to do cool things while in school. And thanks to all my buddies and everyone in lab for good times, even the times that are erased from memory. NTW 


\section{TABLE OF CONTENTS}

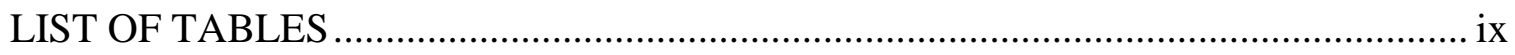

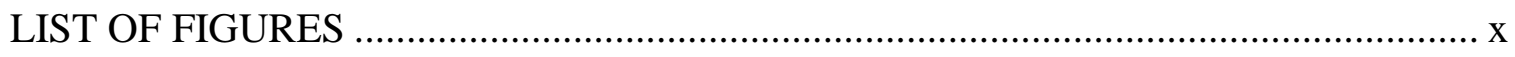

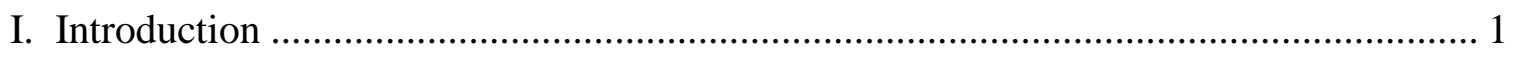

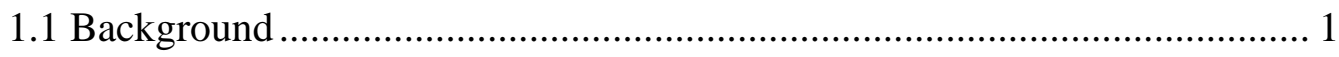

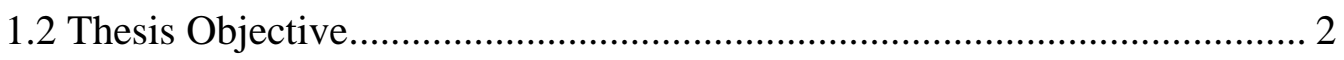

II. Reference Frames and Attitude Representation ......................................................... 4

2.1 Earth Centered Inertial (ECI) .................................................................... 4

2.2 Earth Centered Earth Fixed (ECEF) …………………………..................... 5

2.3 Latitude, Longitude, Altitude (LLA) ………………………………....... 5

2.4 North, East, Down (NED) ....................................................................... 6

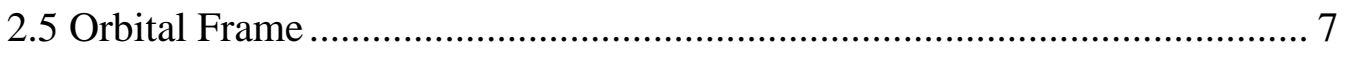

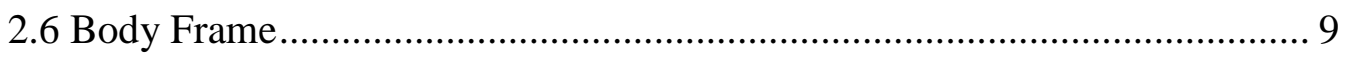

2.7 Direction Cosine Matrix (DCM) ........................................................... 10

2.8 ECI to Orbital Frame Transformation.......................................................... 11

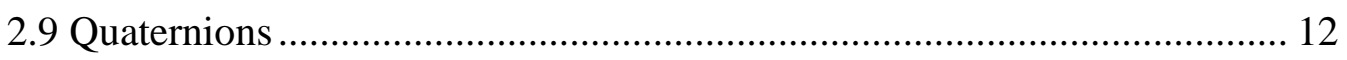

2.10 Attitude Representations........................................................................ 14

III. Spacecraft Rotational Equations of Motion.............................................................. 17

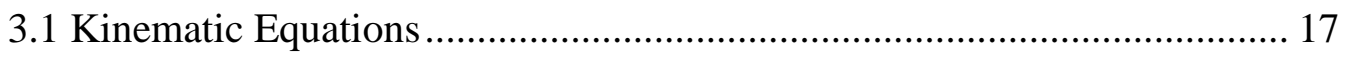

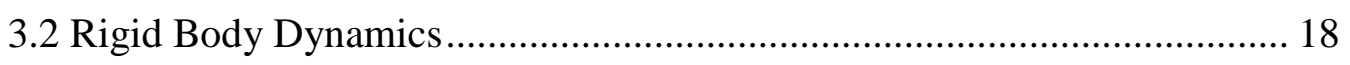

3.2.1 Gravity Gradient Moment................................................................... 20

3.2.1.1 Effect of Orbit Eccentricity.......................................................... 21

3.2.2 Momentum Wheel - Gyrostat Equations............................................... 22

3.2.3 Spherical Damper............................................................................. 24

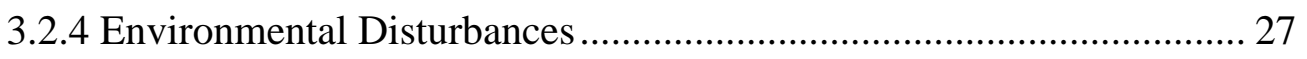

IV. Attitude Sensing and Vector Generation ............................................................... 30 


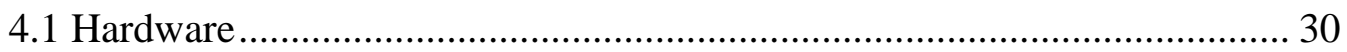

4.2 Orbit Position Knowledge......................................................................... 30

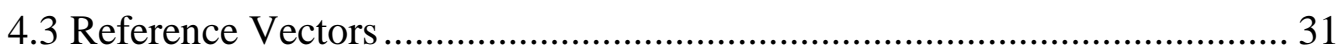

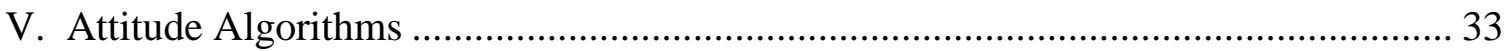

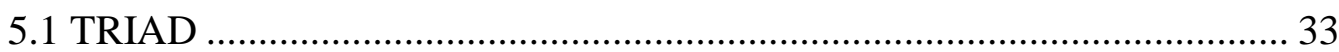

5.2 Extended Kalman Filter .......................................................................... 35

5.2.1 Filter Basis - Dynamics and Measurement Models ............................ 36

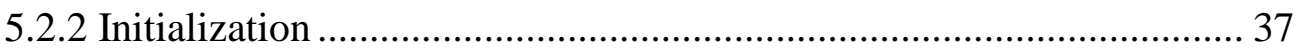

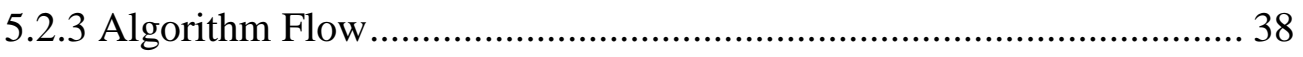

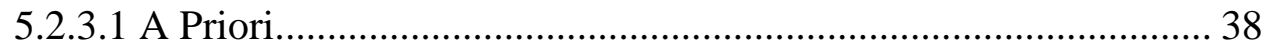

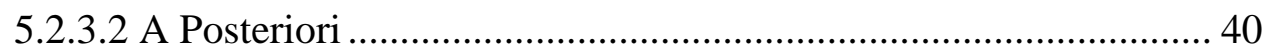

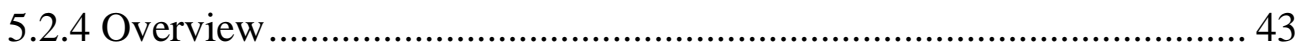

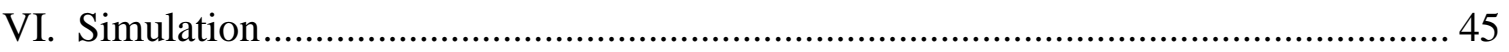

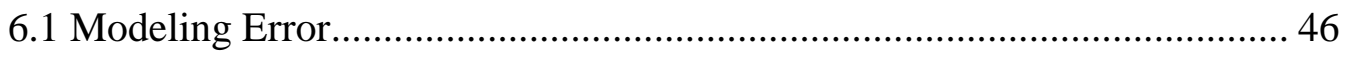

6.2 MATLAB Simulation ........................................................................... 49

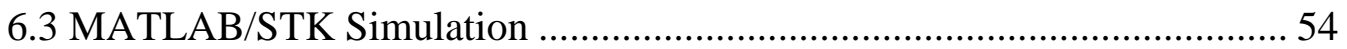

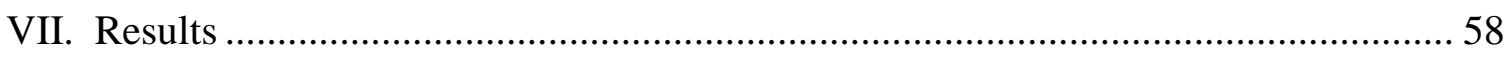

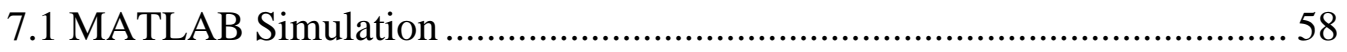

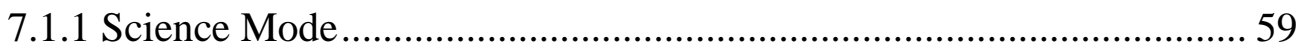

7.1.1.1 Further EKF Analysis for Science Mode ………………………... 69

7.1.1.2 EKF Science Mode Conclusions ................................................ 74

7.1.2 Post Deployment, Pre Gravity Gradient Capture................................... 74

7.1.3 Gravity Gradient Capture / Perturbed Science Mode ……………….... 80

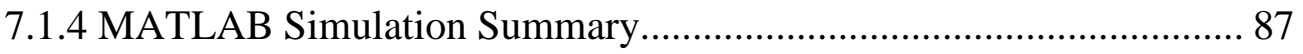

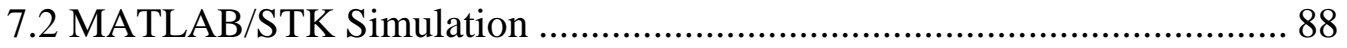

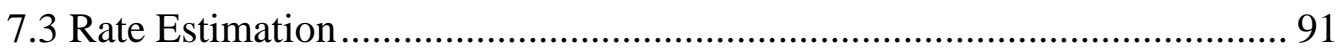

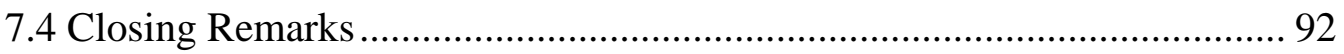


VIII. Conclusion

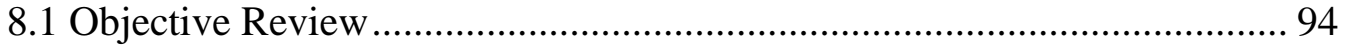

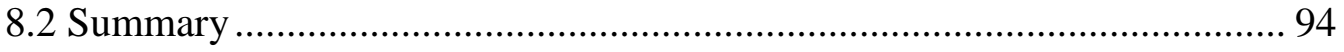

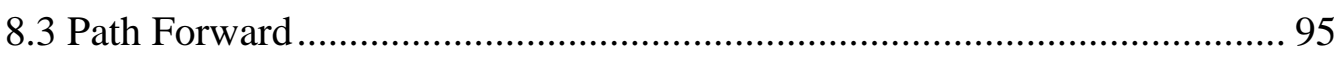

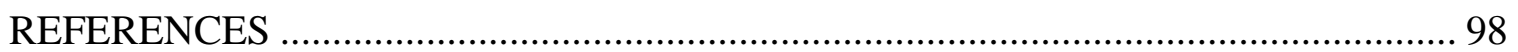

Page viii 


\section{LIST OF TABLES}

Table 1. Average angular error resulting from varying standard deviation random noise inputs..................49

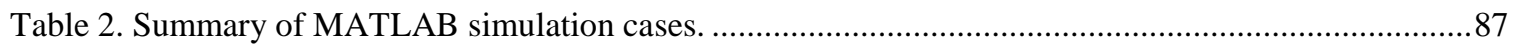

Page ix 


\section{LIST OF FIGURES}

Figure 1. Earth Centered Inertial (ECI) reference frame. Credit to Ryan Sellers. ${ }^{8}$.........................................

Figure 2. Earth Centered Earth Fixed reference frame. Credit to Ryan Sellers. ${ }^{8}$..........................................5

Figure 3. Latitude Longitude Altitude reference frame. Credit to Ryan Sellers. ${ }^{8}$..........................................6

Figure 4. North East Down reference frame. Credit to Ryan Sellers. ${ }^{8}$..........................................................

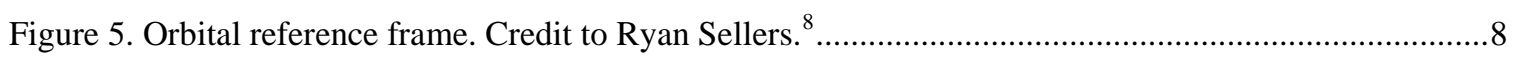

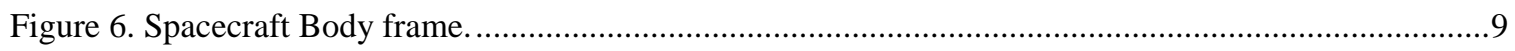

Figure 7. Body frame offset from orbital reference frame. …….................................................................10

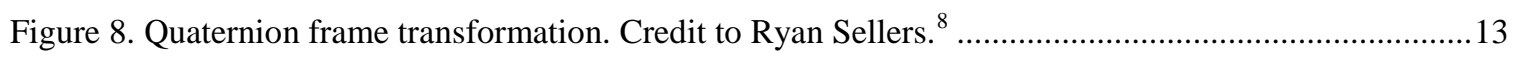

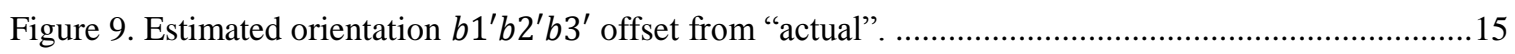

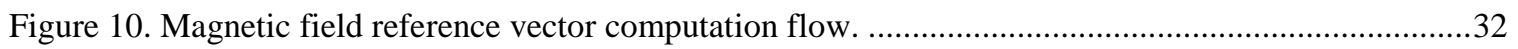

Figure 11. Sun direction reference vector computation flow. ...................................................................

Figure 12. Graphical representation of Extended Kalman Filter...............................................................44

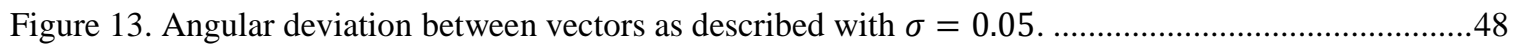

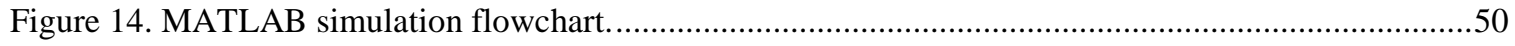

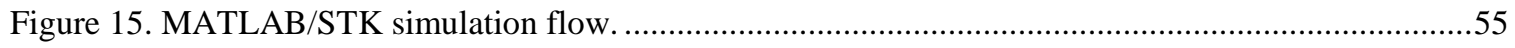

Figure 16. Science mode dynamics, momentum wheel at $500 \mathrm{rpm}$, yaw axis (b1)....................................60

Figure 17. Science mode dynamics, momentum wheel at $500 \mathrm{rpm}$, pitch axis (b3). ...................................60

Figure 18. Science mode dynamics, momentum wheel at $500 \mathrm{rpm}$, roll axis (b2) ......................................61

Figure 19. Science mode dynamics, momentum wheel at 1500 rpm, yaw axis (b1) ...................................62

Figure 20. Science mode dynamics, momentum wheel at $1500 \mathrm{rpm}$, pitch axis (b3). ...............................62

Figure 21. Science mode dynamics, momentum wheel at $1500 \mathrm{rpm}$, roll axis (b2)...................................63

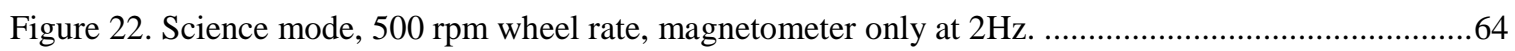

Figure 23. Science mode, $1500 \mathrm{rpm}$ wheel rate, magnetometer only at $2 \mathrm{~Hz}$............................................65

Figure 24. Science mode, $1500 \mathrm{rpm}$ wheel rate, magnetometer and sun sensor at 2Hz, TRIAD.................66

Figure 25. Science mode, $1500 \mathrm{rpm}$ wheel rate, magnetometer and sun sensor at 4Hz, TRIAD.................67

Figure 26. Science mode, $1500 \mathrm{rpm}$ wheel rate, mag/sun sensors at 4Hz, TRIAD, long duration................68 
Figure 27. EKF steady state error resulting from varying input errors...................................................... 70

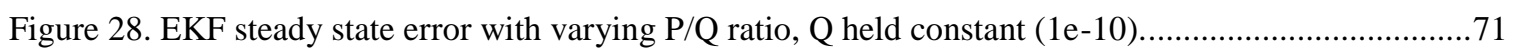

Figure 29. EKF steady state error with varying $\mathrm{P} / \mathrm{Q}$ ratio, $\mathrm{P}$ held constant $(1 \mathrm{e}-10) \ldots \ldots \ldots \ldots \ldots \ldots \ldots \ldots \ldots \ldots \ldots \ldots . . . . .72$

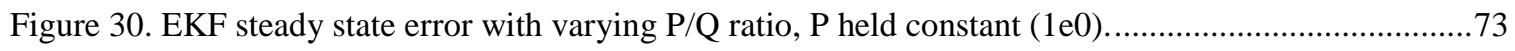

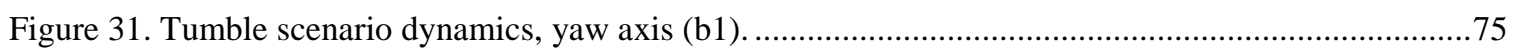

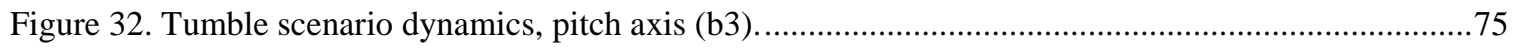

Figure 33. Tumble scenario dynamics, roll axis (b2) ...........................................................................

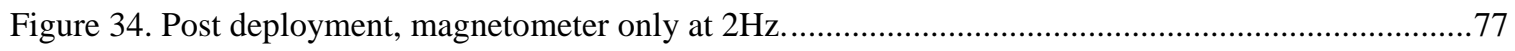

Figure 35. Post deployment, magnetometer and sun sensor at 2Hz. …...................................................78

Figure 36. Post deployment, magnetometer and sun sensor at 2Hz, TRIAD. ............................................79

Figure 37. Gravity Gradient Capture scenario dynamics, yaw axis (b1).................................................... 81

Figure 38. Gravity Gradient Capture scenario dynamics, pitch axis (b3). .................................................. 81

Figure 39. Gravity Gradient Capture scenario dynamics, roll axis (b2) ....................................................82

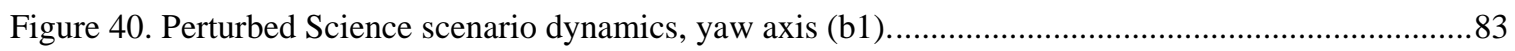

Figure 41. Perturbed Science scenario dynamics, pitch axis (b3) . ........................................................ 83

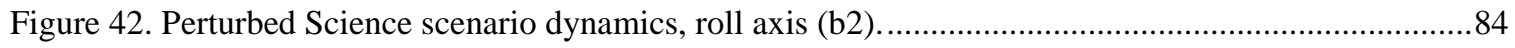

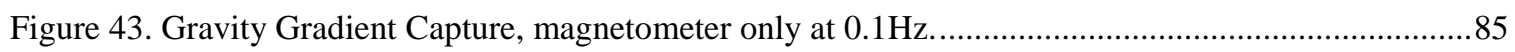

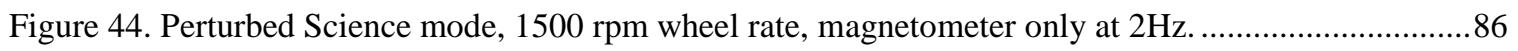

Figure 45. Science mode, STK orbit prop, magnetometer and sun sensor at 2Hz, TRIAD. .........................89

Figure 46. Error between HPOP and SGP4 generated magnetic field vectors. ..........................................90

Figure 47. Body yaw rate and associated error for perturbed science case. ................................................91 


\section{Introduction}

Attitude Determination and Control (ADC) comprises one of the several basic subsystems on most spacecraft. Knowledge and control of spacecraft body orientation in flight can be crucial to a countless number of tasks including orbital maneuvers, perturbation correction, use of instrumentation, and in many cases mission success. Formulation of basic attitude knowledge on a spacecraft usually consists of sensing hardware and the appropriate algorithms to make use of the data taken by them. The hardware types and complexity of these algorithms depend on the level of attitude knowledge required. This thesis is concerned with attitude knowledge estimation for Exocube, a CubeSat class nanosatellite currently under development by PolySat at California Polytechnic State University.

\subsection{Background}

The CubeSat standard was developed jointly by Dr. Jordi Puig-Suari of California Polytechnic State University and Dr. Bob Twiggs of Stanford University in 1999. This standard was created initially as a means for lower budget entities in the aerospace industry to fly satellites, and imposes a somewhat restrictive form factor and mass allotment on satellites following its specification. For the "3U" specification that Exocube follows, this translates to a stowed volume of 10x10x30 cm and a mass allotment around $4 \mathrm{~kg}$. Technological advances and heritage in the nanosatellite field have led to more advanced functionality of these Cubesats in recent years, bringing forth more complicated missions with more stringent requirements on satellite subsystems, 
especially ADC. For Exocube, a summary of mission requirements regarding ADC is as follows:

- The instrument payload shall face the orbit velocity (ram) direction and be aligned with this vector to within $\pm 10^{\circ}$

- Satellite body rates shall remain at or below $0.1 \%$

- Knowledge of attitude shall be within $\pm 5^{\circ}$ of the instrument aperture vector This "instrument aperture vector" corresponds to the body frame roll axis which will be discussed in the next chapter. These requirements are all flowed down from the instrument payload and apply to when it is taking data (or in "science mode", which will also be discussed further). The payload is called EXOS, and is a NASA Goddard developed mass spectrometer that measures an array of atmospheric ion densities. A much more detailed explanation of EXOS and its function can be found in Sellers. ${ }^{8}$

\subsection{Thesis Objective}

The goal of this work is to provide a feasible means of satisfying the attitude knowledge requirement levied on Exocube. This is to be done by research and development of algorithms that will utilize the sensing hardware types selected for this mission, which in typical PolySat fashion comprises small, inexpensive, lower accuracy surface mounted magnetometers and solar angle sensors. The algorithms will be integrated into simulations along with models created for orbit propagation, sensor and reference vector generation, and spacecraft body kinematics/dynamics. These simulations will be run for applicable dynamic cases to verify and prove the feasibility of the algorithms employed, and will also be left as tools for further use in this and future PolySat missions. The 
chapters to come will introduce and explain the attitude reference frames, kinematics and dynamics, reference vector models, and attitude algorithms used, and will then shift to the construction of simulations, which will have their respective results presented and discussed. 


\section{Reference Frames and Attitude Representation}

The attitude of a body is its orientation with respect to a chosen reference frame. The reference frames utilized in the modeling and simulation of spacecraft kinematics and dynamics in this work were derived from Curtis, Kane, Vallado, and Wie. ${ }^{3,6,10,12}$ The following sections explain each type used, expanding more on those more prevalent here.

\subsection{Earth Centered Inertial (ECI)}

The Earth Centered Inertial (ECI) reference frame is fixed with origin at the center of the Earth. The frame is illustrated below.

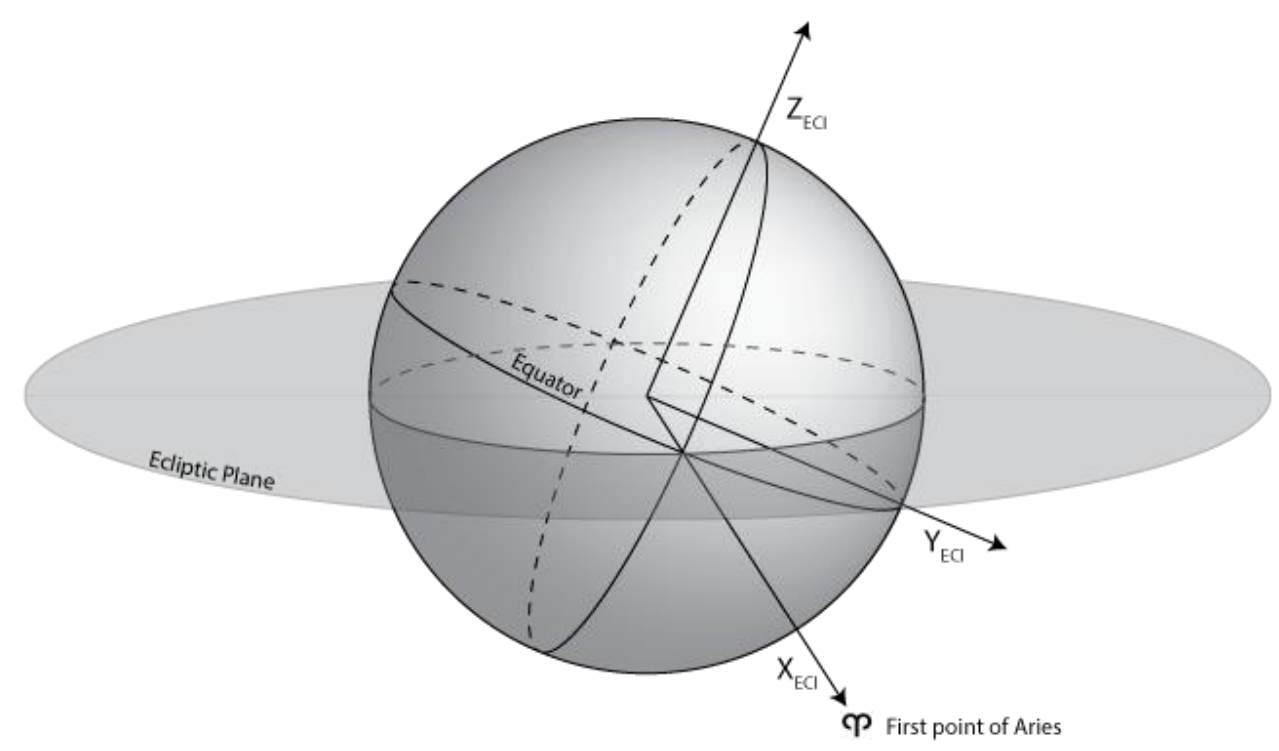

Figure 1. Earth Centered Inertial (ECI) reference frame. Credit to Ryan Sellers. ${ }^{8}$

The x-y plane lies on the equatorial plane with principal direction $X_{E C I}$ aligned with the first point of Aries. $Z_{E C I}$ runs along the North Pole, orthogonal to the equatorial plane, completing the right handed Cartesian coordinate system. Spacecraft orbit radius and velocity vectors are typically expressed in this non-rotating frame for simplicity. 


\subsection{Earth Centered Earth Fixed (ECEF)}

Shown below in Figure 2, the Earth Centered Earth Fixed (ECEF) reference frame is a Cartesian coordinate system that rotates with the Earth.

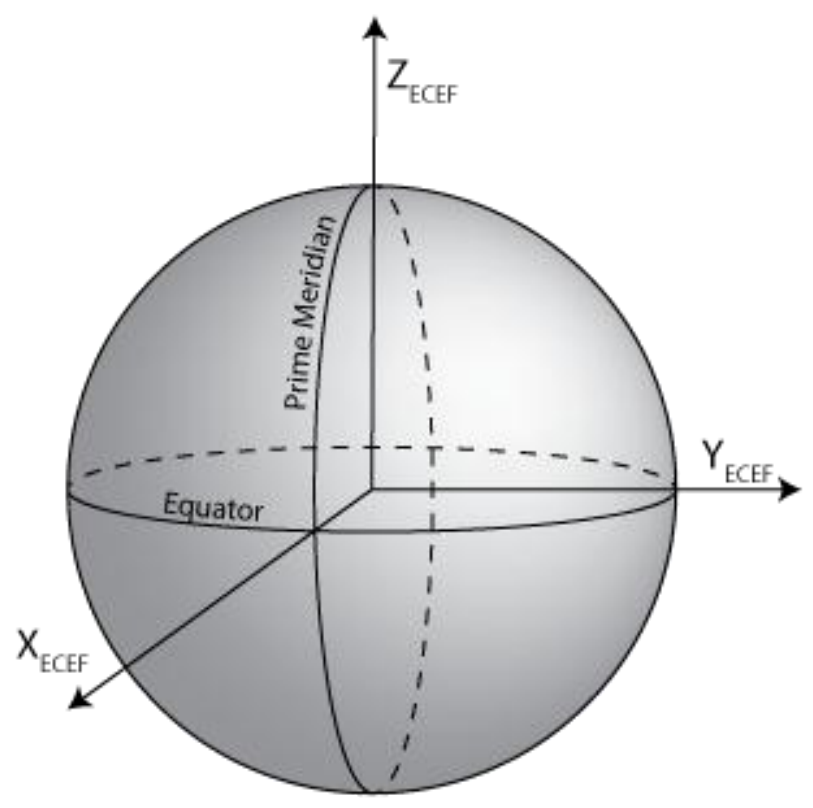

Figure 2. Earth Centered Earth Fixed reference frame. Credit to Ryan Sellers. ${ }^{8}$

The x-y plane is coincident with the equatorial plane, with principal direction $X_{E C E F}$ running along $0^{\circ}$ Latitude, $0^{\circ}$ Longitude. As in the ECI reference frame, this right handed system is completed with $Z_{E C E F}$ aligned with the North Pole, orthogonal to the equatorial plane. In this work, the ECEF coordinate system is used only as an intermediate step in converting ECI to Latitude, Longitude, Altitude (LLA) coordinates.

\subsection{Latitude, Longitude, Altitude (LLA)}

The Latitude, Longitude, Altitude (LLA) reference frame is essentially the ECEF frame expressed in spherical instead of Cartesian coordinates. It is depicted in Figure 3. 


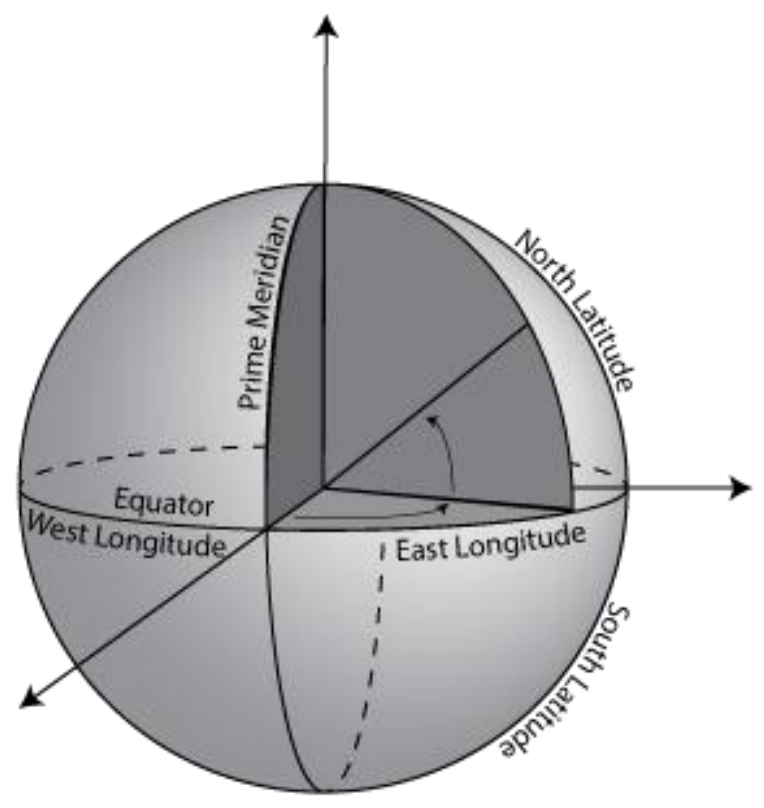

Figure 3. Latitude Longitude Altitude reference frame. Credit to Ryan Sellers. ${ }^{8}$

In this coordinate system, latitude is the angle between the point of interest and the Equator, in which North is positive and South is negative (in some cases the terms colatitude or coelevation are used, in which case the angle complementary to latitude is denoted). Longitude is the angle between the point of interest and the prime meridian in which East is positive and West is negative. Altitude is the distance from the center of the Earth to the point of interest. The use of the LLA frame in this work is confined to coordinates input to a magnetic field model for simulation.

\subsection{North, East, Down (NED)}

The North, East, Down (NED) coordinate system has its origin at the mass center of an orbiting (or flying for aviation's sake) body, as shown below. 


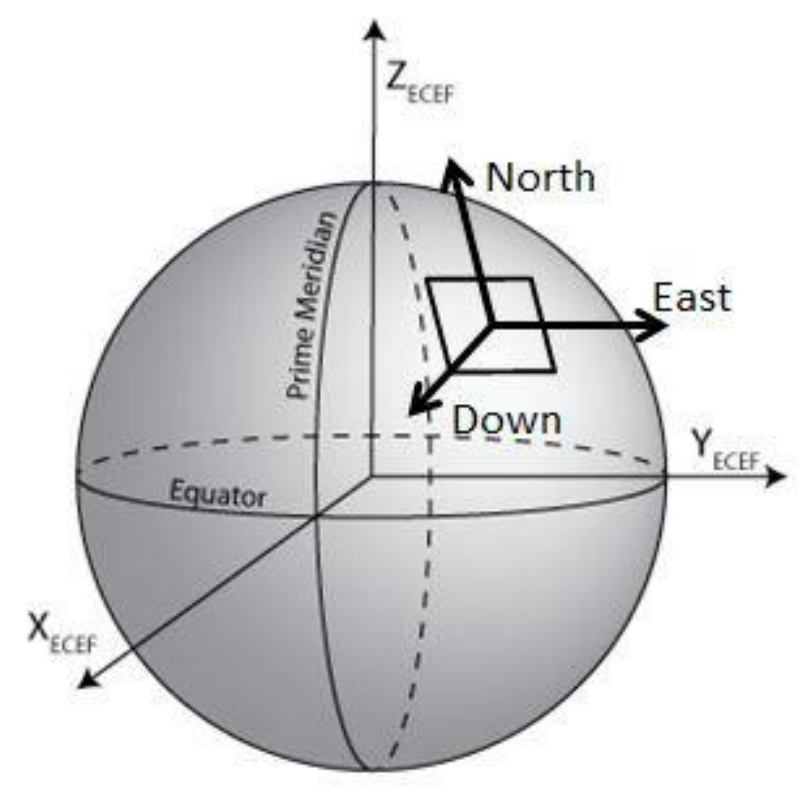

Figure 4. North East Down reference frame. Credit to Ryan Sellers. ${ }^{8}$

Its fundamental plane is tangent to the Earth at the point of interest. Obviously, North represents northern position, East represents eastern position, and Down completes the right handed system, representing vertical position. The only occurrence of this coordinate system in this work lies in the output of the magnetic field model used in simulation.

\subsection{Orbital Frame}

The orbital frame is a rotating reference frame with its origin at the mass center of the orbiting body, as shown in Figure 5. 


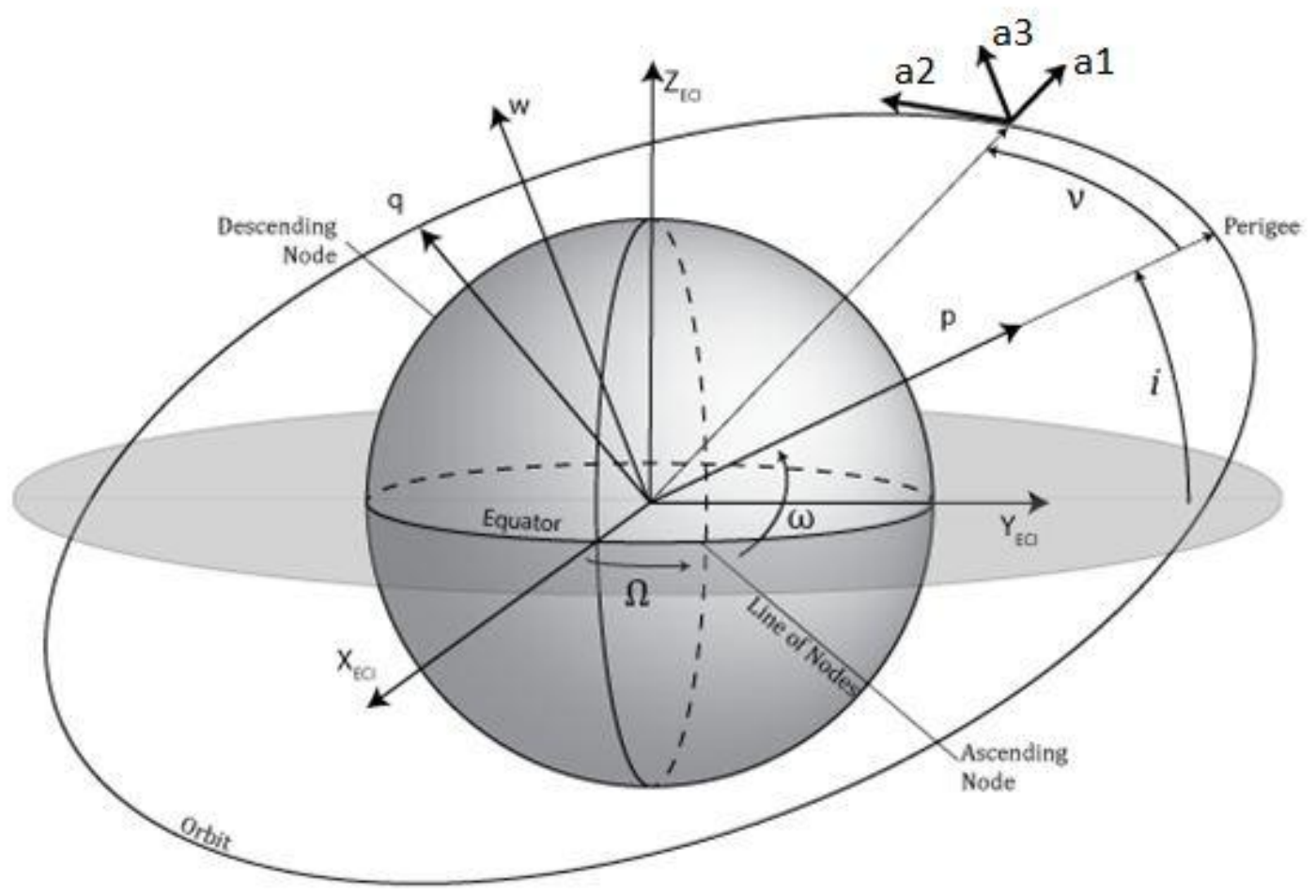

Figure 5. Orbital reference frame. Credit to Ryan Sellers. ${ }^{8}$

The vector $a_{1}$ is aligned with zenith (with the position vector of the body in orbit). $a_{3}$ is aligned with the orbit angular momentum vector (cross track), and $a_{2}$ completes the right handed vector set $\left(a_{2}=a_{3} \times a_{1}\right)$. For circular orbits, the vector $a_{2}$ is aligned with the ram direction (orbit velocity vector). For non-circular orbits, the flight path angle between this "local horizontal" vector and the orbit velocity vector needs to be taken into account, and is described by Vallado. ${ }^{10}$ This reference frame is also called LVLH (Local Vertical Local Horizontal), but it is important to take note of how the axes are aligned in this case, as this is not universal. The axis alignments here are consistent with those of Kane. ${ }^{6}$ 


\subsection{Body Frame}

The body frame has its origin at the mass center of the orbiting body and axes aligned with the body's principle moments of inertia. It is denoted in this work as shown below.

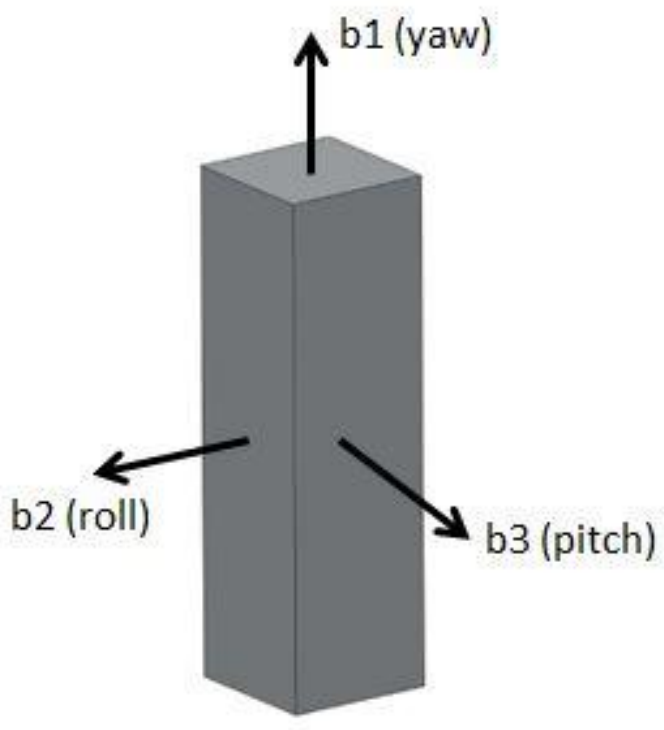

Figure 6. Spacecraft Body frame.

The convention used to label theses axes is somewhat arbitrary, but was chosen as such here so that the body axes will be aligned with their corresponding axes in the orbital frame when Exocube is in its nominal orientation in flight. In that case $b_{1}$ would align with $a_{1}$ (zenith), $b_{2}$ with $a_{2}$, and $b_{3}$ with $a_{3}$ (cross-track). This was done because the attitude representation chosen in this work is the orientation of this body frame with respect to the previously presented orbital reference frame. Yaw, roll, and pitch axes were selected to correspond with $b_{1}, b_{2}$, and $b_{3}$ respectively, as shown in Figure 6 . 


\subsection{Direction Cosine Matrix (DCM)}

A direction cosine matrix (DCM) is a rotation matrix that utilizes direction cosines. These direction cosines describe the projection of one frame onto another. Take for instance the aforementioned body frame and orbital frame, depicted in Figure 7 below.

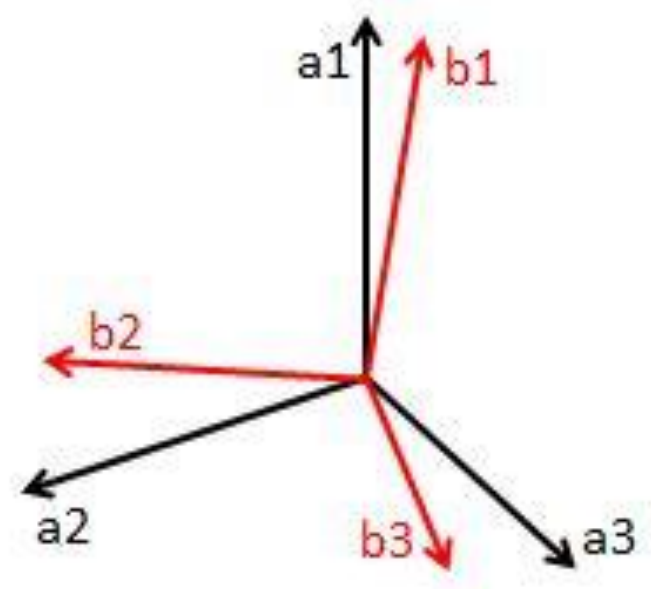

Figure 7. Body frame offset from orbital reference frame.

In order to represent body orientation, or attitude, with respect to the orbital reference frame (or any reference frame for that matter), a DCM can be used. This frame rotation is denoted as

$$
b=C a
$$

in which $b$ is a row matrix containing the basis vectors describing the body frame, $a$ is a row matrix containing the basis vectors describing the orbital reference frame, and $C$ is a 3x3 DCM. Expanding Equation (1) yields 


$$
\left[\begin{array}{l}
b_{1} \\
b_{2} \\
b_{3}
\end{array}\right]=\left[\begin{array}{lll}
C_{11} & C_{12} & C_{13} \\
C_{21} & C_{22} & C_{23} \\
C_{31} & C_{32} & C_{33}
\end{array}\right]\left[\begin{array}{l}
a_{1} \\
a_{2} \\
a_{3}
\end{array}\right]=\left[\begin{array}{l}
C_{11} a_{1}+C_{12} a_{2}+C_{13} a_{3} \\
C_{21} a_{1}+C_{22} a_{2}+C_{23} a_{3} \\
C_{31} a_{1}+C_{32} a_{2}+C_{33} a_{3}
\end{array}\right]
$$

The components of $C$ are the direction cosines describing the projection of the body frame vectors $b_{1}, b_{2}$, and $b_{3}$ onto the orbital frame vectors $a_{1}, a_{2}$, and $a_{3}$. The use of a DCM as such can therefore transform a vector from one reference frame to another (in this example orbital frame to body frame). A more detailed explanation of direction cosine matrices can be found in Curtis, Kane, Wie, and Sidi. ${ }^{3,6,9,12}$

\subsection{ECI to Orbital Frame Transformation}

Aside from the body-orbital representation of attitude, another common frame transformation used in this thesis is from the ECI to orbital reference frame. This is also done with a DCM, using orbit position and velocity (or R and V) vectors to form the appropriate direction cosines. The direction cosines are formed by projecting the ECI frame $\mathrm{R}$ and $\mathrm{V}$ vectors into orbit frame basis vectors as follows:

$$
a_{1}=\frac{R}{\|R\|}
$$

(zenith unit vector)

$$
a_{3}=\frac{R \times V}{\|R \times V\|}
$$

(cross-track unit vector)

$$
a_{2}=a_{3} \times a_{1}
$$

(local horizontal unit vector, completes right handed vector set) 
The appropriate DCM is then formed by augmenting the three row vectors as

$$
C_{E C I}^{\text {orbital }}=\left[\begin{array}{l}
a_{1} \\
a_{2} \\
a_{3}
\end{array}\right]
$$

The DCM formed can then transform an ECI frame vector to the orbital frame as such

$$
a_{\text {orbital }}=C_{E C I}^{\text {orbital }} a_{E C I}
$$

The variable $C$ will denote a DCM in this paper from this point on.

\subsection{Quaternions}

Although more difficult to grasp intuitively, quaternions provide a means of describing the orientation of a body frame in three-dimensional space that avoids the singularities encountered by Euler angle and yaw-pitch-roll sequence rotations. A quaternion is defined by a vector component and a scalar component as

$$
q=\left[\begin{array}{c}
\vec{q} \\
q_{4}
\end{array}\right]=\left[\begin{array}{l}
q_{1} \\
q_{2} \\
q_{3} \\
q_{4}
\end{array}\right]
$$

where $q_{1}, q_{2}$, and $q_{3}$ are the vector components and $q_{4}$ is the scalar component. This notation is very common, but nonetheless many texts define a quaternion with the scalar component followed by the vector component, so it is important to note its form as used here for consistency. The simulations and algorithms in this work all use unit quaternions, which are subject to the constraint $\|q\|=1$. It follows that 


$$
\vec{q}=\sin \frac{\theta}{2} \hat{u} \quad q_{4}=\cos \frac{\theta}{2}
$$

Here $\hat{u}$ is the unit vector along the Euler axis around which the reference frame is rotated to the body frame, and $\theta$ is the Euler principal rotation angle, as described by Curtis. ${ }^{3}$ This rotation is shown in Figure 8 below.

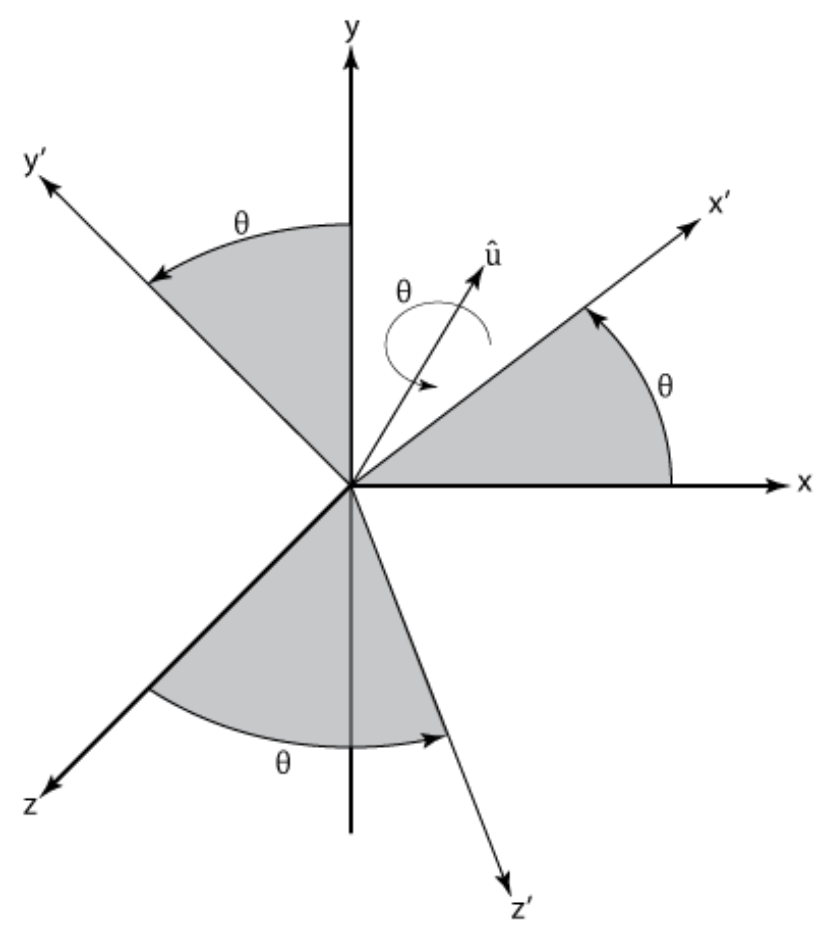

Figure 8. Quaternion frame transformation. Credit to Ryan Sellers. ${ }^{8}$

More generally, this Euler axis is defined by one of Euler's theorems, which states that any two Cartesian coordinate frames are related by a unique rotation about a single line through their common origin. ${ }^{3}$ For a derivation of quaternions following this theorem, see Curtis. ${ }^{3}$ Because quaternions are four-dimensional vectors, the product of two quaternions is 


$$
q_{A} \otimes q_{B}=\left[\begin{array}{cccc}
q_{A_{4}} & q_{A_{3}} & -q_{A_{2}} & -q_{A_{1}} \\
-q_{A_{3}} & q_{A_{4}} & q_{A_{1}} & -q_{A_{2}} \\
q_{A_{2}} & -q_{A_{1}} & q_{A_{4}} & -q_{A_{3}} \\
q_{A_{1}} & q_{A_{2}} & q_{A_{3}} & q_{A_{4}}
\end{array}\right] q_{B}
$$

This quaternion product can be utilized to perform frame rotations. A final note here on quaternions is with regards to the formation of a DCM relating a reference frame and body frame. This DCM is created using quaternions as ${ }^{11}$

$$
C=\left[\begin{array}{ccc}
1-2\left(q_{2}{ }^{2}+q_{3}{ }^{2}\right) & 2\left(q_{1} q_{2}+q_{3} q_{4}\right) & 2\left(q_{1} q_{3}-q_{2} q_{4}\right) \\
2\left(q_{1} q_{2}-q_{3} q_{4}\right) & 1-2\left(q_{1}{ }^{2}+q_{3}{ }^{2}\right) & 2\left(q_{2} q_{3}+q_{1} q_{4}\right) \\
2\left(q_{1} q_{3}+q_{2} q_{4}\right) & 2\left(q_{2} q_{3}-q_{1} q_{4}\right) & 1-2\left(q_{1}{ }^{2}+q_{2}{ }^{2}\right)
\end{array}\right]
$$

This DCM scheme is used here to relate orbital frame vectors to the body frame; transformations for this are carried out as discussed in Section 2.7 Direction Cosine Matrix (DCM).

\subsection{Attitude Representations}

In the simulations to come, attitude knowledge is presented as the error between two quaternions - one simulated as "actual" spacecraft attitude, and the other simulated as the estimated attitude (these attitudes are body orientation with respect to an orbital reference frame). The error is formulated through a quaternion product $q_{\text {actual }} \otimes q_{\text {estimate }}$ as presented in Equation (10), and can be visualized as a rotation between the "actual" body frame and the estimated body frame. Although quaternions are used here for all simulations involving spacecraft kinematics, dynamics, and attitude estimation algorithms, they are not used directly as a final representation of attitude knowledge.

Standard angles are used instead, denoting the total angular deviation between 
corresponding axes of the "actual" and estimated body frames. These angles are derived from a DCM that is formulated from the calculated error quaternion using the convention shown in Equation (11). Angular deviation corresponding to the body yaw, roll, and pitch axes are then extracted from the DCM as

$$
\begin{aligned}
& \operatorname{yaw}_{d e v}=\cos ^{-1}\left(C_{11}\right) \\
& \operatorname{roll}_{d e v}=\cos ^{-1}\left(C_{22}\right) \\
& \text { pitch }_{d e v}=\cos ^{-1}\left(C_{33}\right)
\end{aligned}
$$

These angular deviations for body yaw, roll, and pitch (or $b_{1}, b_{2}$, and $b_{3}$ ) axes correspond to the offset of the two frames shown below in Figure 9.

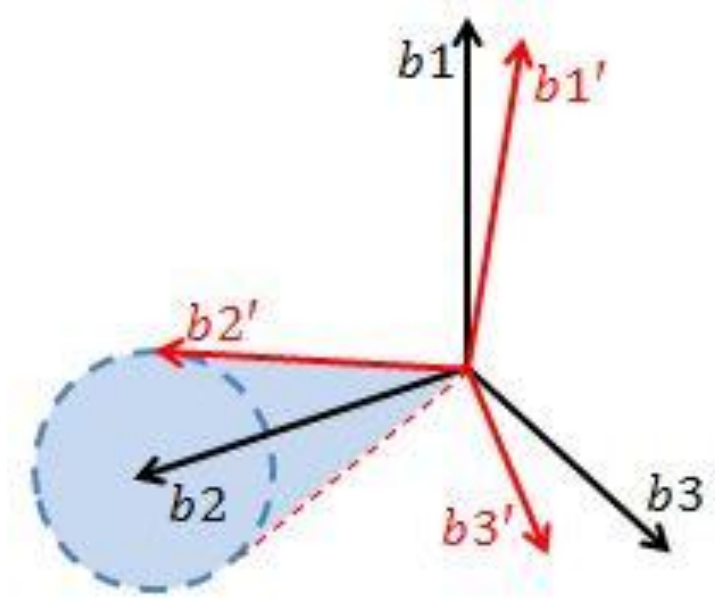

Figure 9. Estimated orientation $b 1^{\prime} b 2^{\prime} b 3^{\prime}$ offset from "actual".

This representation scheme was chosen to more directly follow the Exocube attitude knowledge requirement of \pm 5 degrees in the ram direction, which can be visualized with the "cone" of angular deviation about each axis formed by the 3-axis frame offset 
depicted. Because these angles are products of the arccosine function, they will always be positive and serve to provide the magnitude of angular error (attitude knowledge).

All results pertaining to spacecraft dynamics are represented in the simpler standard yawpitch-roll sequence representation of angles. These angles are derived from the bodyorbital frame DCM formulated by Equation (11) by means of an algorithm outlined by Curtis. ${ }^{3}$ It is important to note here that although the algorithm is employed exactly as presented by Curtis, the resultant yaw, pitch, and roll angles do not correspond with the yaw, pitch, roll axes in this work. This is due to the implementation of different axes alignments and in no way affects the calculated spacecraft kinematics/dynamics. 


\section{Spacecraft Rotational Equations of Motion}

The following will serve as a brief explanation and overview of the equations formulated in this work for use in the modeling/simulation of spacecraft attitude in a Low Earth Orbit environment.

\subsection{Kinematic Equations}

The standard time derivative of a quaternion is given $b y^{3}$

$$
\dot{q}=\frac{1}{2}\left[\begin{array}{cccc}
0 & \omega_{3} & -\omega_{2} & \omega_{1} \\
-\omega_{3} & 0 & \omega_{1} & \omega_{2} \\
\omega_{2} & -\omega_{1} & 0 & \omega_{3} \\
-\omega_{1} & -\omega_{2} & -\omega_{3} & 0
\end{array}\right] q
$$

in which $\omega_{1}, \omega_{2}$, and $\omega_{3}$ are the spacecraft angular velocities in the body frame. Because the spacecraft states are propagated forward with respect to an orbital reference frame in this work, the motion of the orbit needs to be accounted for in the angular rates propagated. This rewrites Equation (13) as

$$
\dot{q}=\frac{1}{2}\left[\begin{array}{cccc}
0 & \omega_{3}+\dot{\theta} & -\omega_{2} & \omega_{1} \\
-\omega_{3}-\dot{\theta} & 0 & \omega_{1} & \omega_{2} \\
\omega_{2} & -\omega_{1} & 0 & \omega_{3}-\dot{\theta} \\
-\omega_{1} & -\omega_{2} & -\omega_{3}+\dot{\theta} & 0
\end{array}\right] q
$$

where $\dot{\theta}$ is the rate of change of true anomaly of the orbit (angular rate about orbital $a_{3}$ axis). It is calculated with

$$
\dot{\theta}=\frac{n a^{2} \sqrt{1-e^{2}}}{R^{2}}
$$


where $a$ is the orbit semimajor axis, $e$ is orbit eccentricity, and $R$ is orbit radius. For circular orbits, Equation (15) reduces to the constant orbit rate, or mean motion $n$. Orbit radius is updated with ${ }^{5}$

$$
R=\frac{a\left(1-e^{2}\right)}{(1+e \cos \theta)}
$$

The two preceding equations are included in dynamic simulations that can account for eccentric orbits. Equation (15) provides the differential equation for orbit true anomaly, while Equation (16) provides an update for the former at each iteration.

\subsection{Rigid Body Dynamics}

The formulation of the rotational dynamics equations used in this thesis is gathered largely from Curtis, Kane, and Wie. ${ }^{3,6,12}$ More extensive derivations for these equations can be found in any of those references, as the following explanation will attempt to be more concise. These dynamics assume a rigid spacecraft body. Any additional assumptions will be stated where necessary.

Euler's equations for a body in pure rotation state that the net moment on the body is equal to the time rate derivative of its angular momentum, or

$$
M_{n e t}=\dot{H}
$$

where $M_{\text {net }}$ is the net moment and $H$ is its angular momentum. The total time derivative of angular momentum can be expanded as 


$$
M_{n e t}=\dot{H}_{r e l}+\Omega \times H
$$

Here $\dot{H}_{r e l}$ is the time derivative of angular momentum with respect to the spacecraft body, and $\Omega$ is a vector of the angular velocities of a co-moving frame. For a co-moving frame that's rigidly attached to the spacecraft body (moving axes are principle axes of inertia), this vector is simply the angular velocities of the spacecraft body. Using the fact that $H=I \omega$ ( $I$ is the spacecraft diagonal inertia tensor), Equation (18) becomes

$$
M_{n e t}=I \dot{\omega}+\omega \times(I \omega)
$$

Solving the cross product and expanding Equation (19) gives equations of motion for each of the body axes as follows

$$
\begin{aligned}
& M_{1_{\text {net }}}=I_{1} \dot{\omega}_{1}+\left(I_{3}-I_{2}\right) \omega_{2} \omega_{3} \\
& M_{2_{\text {net }}}=I_{2} \dot{\omega}_{2}+\left(I_{1}-I_{3}\right) \omega_{1} \omega_{3} \\
& M_{3_{\text {net }}}=I_{3} \dot{\omega}_{3}+\left(I_{2}-I_{1}\right) \omega_{1} \omega_{2}
\end{aligned}
$$

Rearranging terms to solve for angular velocity derivatives gives

$$
\begin{aligned}
& \dot{\omega}_{1}=I_{1}^{-1}\left(M_{1_{n e t}}+\left(I_{2}-I_{3}\right) \omega_{2} \omega_{3}\right) \\
& \dot{\omega}_{2}=I_{2}^{-1}\left(M_{2_{n e t}}+\left(I_{3}-I_{1}\right) \omega_{1} \omega_{3}\right) \\
& \dot{\omega}_{3}=I_{3}^{-1}\left(M_{3_{n e t}}+\left(I_{1}-I_{2}\right) \omega_{1} \omega_{2}\right)
\end{aligned}
$$

This form of Euler's equation will be utilized from this point on for dynamics analysis. 


\subsubsection{Gravity Gradient Moment}

Because the gravitational attraction between two bodies varies with the inverse square of the distance between them, there is a variation of the Earth's gravitational field along the volume of an orbiting spacecraft. For a more elongated body like that of Exocube, this causes a moment about the mass center of the body that acts to align its axis of minimum inertia (yaw or $\left.b_{1}\right)$ with the local vertical ( $a_{1}$ in the orbital reference frame). This moment obviously vanishes if the two axes are aligned or perfectly perpendicular, but for any angular deviation from that alignment, the induced moment causes the body's minor axis to rotate towards alignment with the local vertical. The body will continue rotating past the local vertical due to its inertia until reversed by the same moment in the opposite direction. What results is a stable undamped oscillation about the roll and pitch axes of the spacecraft ( $b_{2}$ and $b_{3}$ respectively). It is important to note here that this oscillation is only stable for certain ranges of inertia ratios and low eccentricity orbits (ideally circular). For a more detailed analysis and verification that Exocube meets the necessary stability criteria see Sellers. ${ }^{8}$ As presented in most texts, an equation describing the gravity gradient moment acting on a rigid body in a circular orbit is given by

$$
M_{g g}=3 n^{2} \cdot S \times I \cdot S
$$

where $M_{g g}$ is the gravity gradient moment, and $S$ accounts for the attitude of the spacecraft relative to the local vertical through multiplication of an appropriate direction cosine matrix by the direction of the local vertical. Utilizing the orbital frame, it is expressed as 


$$
S=C_{b / o} \hat{l}_{o}
$$

where $C_{b / o}$ is simply the direction cosine matrix relating the body and orbital frames presented earlier by Equation (11), and the term $\hat{\imath}_{o}$ denotes the direction of the orbital frame that is in line with the local vertical. Computing the product in Equation (23) yields

$$
S=C\left[\begin{array}{l}
1 \\
0 \\
0
\end{array}\right]=\left[\begin{array}{l}
C(1,1) \\
C(2,1) \\
C(3,1)
\end{array}\right]
$$

Substituting Equation (24) into Equation (22) and collecting terms for each body axis gives

$$
\begin{aligned}
& M_{g g_{1}}=3 n^{2}\left(I_{3}-I_{2}\right) C(2,1) C(3,1) \\
& M_{g g_{2}}=3 n^{2}\left(I_{1}-I_{3}\right) C(1,1) C(3,1) \\
& M_{g g_{3}}=3 n^{2}\left(I_{2}-I_{1}\right) C(1,1) C(2,1)
\end{aligned}
$$

These equations denote the gravity gradient moment as seen by the body axes. To account for them in the rotational motion of a body in orbit, they are simply incorporated in Euler's equations as part of $M_{\text {net }}$.

\subsubsection{Effect of Orbit Eccentricity}

As mentioned previously, the oscillation induced by a gravity gradient moment on a rigid body can become unstable if the eccentricity of the orbit in question is not small enough. To model this effect, the rate of change in true anomaly of the orbit needs to be taken into 
account, since it is no longer constant with a non-circular orbit. Using Equation (15), Equation (22) is rewritten to account for orbit eccentricity ${ }^{7}$

$$
M_{g g}=3 \frac{\dot{\theta}^{2}}{(1+e \cos \theta)} \cdot S \times I \cdot S
$$

On inspection, it can be seen that for a circular orbit the second term in Equation (26) reduces to the mean motion squared as it is in Equation (22). Using this more expanded form of gravity gradient torque calculation in a numeric integrator allows the dynamic model of the rigid body to take into account the effects of an eccentric orbit.

\subsubsection{Momentum Wheel - Gyrostat Equations}

Although proper utilization of the gravity gradient moment on a spacecraft can place it in a nadir-pointing orientation as is desired for Exocube, the problem of yaw axis $\left(b_{1}\right)$ stabilization still remains. One method to solve this which is going to be employed on Exocube is the use of a momentum wheel along or parallel to the body pitch axis $\left(b_{3}\right)$. At constant speed in this orientation, the wheel will provide gyroscopic stiffness to the rigid body in its other two axes. The dynamics for an orbiting gyrostat are described by the following modification to Equation (19) which includes the angular velocity and inertia of the momentum wheel

$$
M_{n e t}=I \dot{\omega}+\omega \times\left(I \omega+J_{w} \omega_{w}\right)
$$

Here $J_{w}$ and $\omega_{w}$ are the wheel diagonal inertia tensor and angular rate respectively. To simplify the dynamics of the system, it is assumed here that the wheel is mounted exactly at the origin of the pitch axis $\left(b_{3}\right)$, in this case making the wheel inertia tensor zeros 
except for $J_{w}(3,3)$ which is simply the wheel's moment of inertia about its axis of rotation. Another simplification made is the fact that the momentum wheel constant angular rate is used instead of its angular rate relative to the rigid body. This assumption was deemed acceptable due to the fact that for the case of the Exocube mission, the wheel will be operating at an angular rate at least four orders of magnitude greater than that of the satellite in nominal operation. Using these assumptions and solving Equation (27) for the angular rate differential equations for each body axis yields

$$
\begin{gathered}
\dot{\omega}_{1}=I_{1}^{-1}\left(M_{1_{n e t}}+\left(I_{2}-I_{3}\right) \omega_{2} \omega_{3}-J_{w} \omega_{w} \omega_{2}\right) \\
\dot{\omega}_{2}=I_{2}{ }^{-1}\left(M_{2_{n e t}}+\left(I_{3}-I_{1}\right) \omega_{1} \omega_{3}+J_{w} \omega_{w} \omega_{1}\right) \\
\dot{\omega}_{3}=I_{3}{ }^{-1}\left(M_{3_{n e t}}+\left(I_{1}-I_{2}\right) \omega_{1} \omega_{2}\right)
\end{gathered}
$$

On inspection of Equation (28) one can clearly see the gyroscopic effect that the pitch axis $\left(b_{3}\right)$ momentum wheel has on the yaw and roll axes $\left(b_{1}\right.$ and $\left.b_{3}\right)$. Because the wheel is always modeled at a constant angular rate in this case, there is no additional moment induced and the body moments of inertia are unaffected by wheel rotation. For a more generalized form of equations concerning gyrostats, see Kane. ${ }^{6}$ As a final note in this section, it should be known that dynamics simulations conducted in this work revealed a secondary frequency of spacecraft body oscillation about the yaw and roll axes with the use of a momentum wheel along the pitch axis. This "jitter" is induced by the gyroscopic coupling described in Equation (28), and is present with the use of the momentum wheel when the spacecraft body has rate disturbances applied to its axes, which it inevitably will in flight. The amplitude and frequency of these oscillations are dependent on the momentum wheel rate and momentum wheel inertia relative to the body, and 
interestingly enough were found to be independent of the magnitude of body rate disturbance(s).

\subsubsection{Spherical Damper}

The effect of a viscous damper was also looked into and modeled in this work, although it is somewhat of a digression from the thesis scope. A damper would be useful in that it has the potential to passively reduce spacecraft oscillation to the point at which the implemented magnetorquers would not be necessary (with respect to the Exocube attitude control requirement) to slow the spacecraft body rates. Decreased use of magnetorquers for Exocube would mean more time for its payload instrument to operate and less interrupted use of the planned attitude estimation routine, which utilizes magnetometers for attitude sensing. In this case a spherical damper was modeled due to its relative simplicity. This type of damper consists of a sphere (oddly enough) within a larger sphere (or any housing with an inner spherical geometry) with fluid filling the void between them. The two main parameters driving the effectiveness of this type of damper are its inertia and viscous damping coefficient. The former is a function of its size and material composition, while the latter is a function of the viscosity of the fluid used as well as the fluid film thickness. Because the inner sphere is free to move within the fluid inside the spherical damper housing, spacecraft body kinetic energy can be dissipated by conversion to heat energy via the shear force of the fluid induced by relative movement between the body and damper. This would ideally reduce spacecraft body rates over a period of time dictated by the aforementioned damper characteristics as well as spacecraft oscillatory rates. The dynamical model for the fluid damper effect employs an equation for an 
induced damping moment based on viscous damping coefficient and damper angular velocities relative to those of the spacecraft body, as well as differential equations for the damper rates themselves. The damper speed and inertia are not included in Euler's equations in the same manner as the momentum wheel was, since their products were assumed negligibly small for a useable geometry and mass in the case of Exocube. It was also assumed for simplicity that the damper is located at the spacecraft body center of mass. The moment on the spacecraft body induced by the damper is given by

$$
M_{d a m p}=-c\left[\begin{array}{l}
\omega_{1}-\omega_{1} \\
\omega_{2}-\omega_{2 d} \\
\omega_{3}-\omega_{3_{d}}
\end{array}\right]
$$

in which $c$ is the viscous damping coefficient, and the terms $\omega_{1_{d}}, \omega_{2 d}$, and $\omega_{3_{d}}$ denote the angular velocity of the damper in the body axes. A velocity difference is used as an expanded form of relative velocities for clarity here and in the upcoming differential equation for damper angular velocities, which in compact form is

$$
J_{d} \dot{\omega}_{\text {damp,rel }}=c \omega_{\text {damp }, r e l}
$$

$J_{d}$ is the inertia tensor for the damper, which in this case is a diagonal matrix with each nonzero element set to the inertia of the sphere used. $\omega_{\text {damp,rel }}$ is the angular velocity vector of the damper relative to the spacecraft body and can be expressed as in Equation (29). $\dot{\omega}_{\text {damp,rel }}$ is the time derivative of the damper angular velocity relative to the body and is expanded in a manner very similar to the derivation of Euler's equations for the body rotational rates. It is as follows: 


$$
\dot{\omega}_{d a m p, r e l}=\frac{d H_{d a m p}}{d t}+\omega \times H_{d a m p}
$$

Here $d H_{d a m p} / d t$ is the time derivative of the damper angular momentum, or its inertia tensor multiplied by its angular acceleration vector. $\omega$ is the body frame angular velocity vector, and $H_{\text {damp }}$ is the damper angular momentum, or its inertia tensor multiplied by its angular velocity vector. Using these definitions for angular momentum and its time derivative and solving the cross product in Equation (31) results in

$$
\dot{\omega}_{\text {damp }, r e l}=\left[\begin{array}{l}
\dot{\omega}_{1_{d}}+\left(\omega_{2} \omega_{3_{d}}-\omega_{3} \omega_{2_{d}}\right) \\
\dot{\omega}_{2_{d}}+\left(\omega_{3} \omega_{1_{d}}-\omega_{1} \omega_{3_{d}}\right) \\
\dot{\omega}_{3_{d}}+\left(\omega_{1} \omega_{2_{d}}-\omega_{2} \omega_{1_{d}}\right)
\end{array}\right]
$$

Substituting this expression into Equation (30) and solving for the damper angular acceleration terms then gives the damper angular velocity differential equations corresponding to each body axis as

$$
\begin{aligned}
& \dot{\omega}_{1_{d}}=\frac{c}{J_{d}}\left(\omega_{1}-\omega_{1_{d}}\right)+\left(\omega_{3} \omega_{2_{d}}-\omega_{2} \omega_{3_{d}}\right) \\
& \dot{\omega}_{2_{d}}=\frac{c}{J_{d}}\left(\omega_{2}-\omega_{2_{d}}\right)+\left(\omega_{1} \omega_{3_{d}}-\omega_{3} \omega_{1_{d}}\right) \\
& \dot{\omega}_{3_{d}}=\frac{c}{J_{d}}\left(\omega_{3}-\omega_{3_{d}}\right)+\left(\omega_{2} \omega_{1_{d}}-\omega_{1} \omega_{2_{d}}\right)
\end{aligned}
$$

These equations are used in a numeric integrator along with the kinematic differential equations and differential equations for body angular velocity to propagate spacecraft attitude given an initial state. Dynamics analysis showed that incorporation of this specific damper model completely diminished the momentum wheel induced jitter 
mentioned in the previous section over the course of $\sim 80$ orbits, but did not significantly impact overall steady state oscillation amplitude. A damper modeled at a more realistic location offset from the body mass center would likely have more of an effect, as its inertia relative to the mass center would increase.

For the sake of completeness, the differential equations describing rotational motion of the rigid body as used in the dynamics simulations in this work are presented here using the constant speed momentum wheel as well as gravity gradient and spherical damper moments.

$$
\begin{gathered}
\dot{\omega}_{1}=I_{1}^{-1}\left(M_{g g_{1}}+M_{d a m p_{1}}+M_{d_{1}}+\left(I_{2}-I_{3}\right) \omega_{2} \omega_{3}-J_{w} \omega_{w} \omega_{2}\right) \\
\dot{\omega}_{2}=I_{2}^{-1}\left(M_{g g_{2}}+M_{d a m p_{2}}+M_{d_{2}}+\left(I_{3}-I_{1}\right) \omega_{1} \omega_{3}+J_{w} \omega_{w} \omega_{1}\right) \\
\dot{\omega}_{3}=I_{3}{ }^{-1}\left(M_{g g_{3}}+M_{\text {damp }_{3}}+M_{d_{3}}+\left(I_{1}-I_{2}\right) \omega_{1} \omega_{2}\right)
\end{gathered}
$$

The terms $M_{g g_{x}}$ and $M_{d a m p_{x}}$ correspond to the vector indices of Equation (26) and Equation (29) respectively, while the terms $M_{d_{x}}$ are added in to account for any additional induced environmental disturbance moments (due to atmospheric drag, solar radiation pressure, residual magnetic dipole) should they be modeled.

\subsubsection{Environmental Disturbances}

For a body in low earth orbit, the main sources of attitude disturbance are gravity gradient, atmospheric drag, solar radiation pressure, and interactions with the Earth's magnetic field. Given Exocube's geometry, mass properties, material composition, and currently anticipated altitude of 400-670 km, moments from gravity gradient and drag 
effects are the largest anticipated in orbit. Because in the case of Exocube the gravity gradient moment is utilized as a means of passive control, it was modeled more in depth previously. The moment on a body in orbit caused by aerodynamic drag can be expressed by

$$
M_{\text {drag }}=r_{c p / c m} \times\left(F_{d r a g} \frac{v}{\|v\|}\right)
$$

where $r_{c p / c m}$ is the position vector of the body center of pressure with respect to center of mass and is crossed with the drag force multiplied by a normalized orbit velocity vector. The drag force is given by

$$
F_{\text {drag }}=\frac{1}{2} \rho V^{2} A C_{D}
$$

in which $\rho$ is atmospheric density, $V$ is the magnitude of the orbit velocity, $A$ is the body cross sectional area, and $C_{D}$ is drag coefficient.

In this work, the modeling of environmental moments aside from the gravity gradient effect was only used as a means to explore the robustness of the attitude estimation algorithms developed. For this reason, a very simplified version of Equation (35) was modeled assuming a drag disturbance only about the body pitch axis $\left(b_{3}\right)$. Atmospheric density was varied sinusoidally through each orbit, reaching a user defined maximum value at perigee and minimum value at apogee. This drag model did not take into account the Earth's oblateness or motion of the atmosphere with respect to the orbit. Solar radiation pressure was not modeled due to the fact that the moments generated would be at least one to two orders of magnitude lower than that of drag and gravity gradient, and 
residual magnetic dipole effects were accounted for with the pulsing of the control magnetorquers in use as done by Sellers. ${ }^{8}$ For a more detailed account of the environmental disturbances discussed here see Modi, Sellers. ${ }^{7,8}$ 


\section{Attitude Sensing and Vector Generation}

This chapter will describe the more general means used by Exocube to gather and propagate data in the space environment from which attitude knowledge is computed (attitude algorithms will be presented further on). Bowen is referenced heavily here. ${ }^{1}$

\subsection{Hardware}

The attitude sensing hardware to be employed on Exocube comprises surface mount magnetometers and solar angle sensors. These sensor types were chosen because they can be utilized to estimate spacecraft attitude within the mission knowledge requirement while remaining inexpensive, small, and easily integrated. Hardware that has been tentatively chosen consists of Honeywell HMC5883L magnetometers and ELMOS E910.86 solar angle sensors. Honeywell claims $1-2^{\circ}$ accuracy for the magnetometer, while the solar angle sensor spec sheets claim a $2.7^{\circ}$ angular resolution. Actual values will not be known until these are tested and calibrated, so these angular uncertainties are increased in simulation for margin.

\subsection{Orbit Position Knowledge}

Orbit position knowledge for Exocube serves two very important needs. Firstly, satellite position must be known for points at which the science instrument payload Exos takes data. Also, both attitude reference vector lookups and/or frame transformations of their outputs (discussed in the following section) require orbit position and velocity vectors at the time of interest. The Simplified General Perturbations Model (SGP4) is the orbit propagation model chosen for use on Exocube, and provides orbit ephemeris taking into consideration atmospheric drag, solar radiation pressure, gravitational effects due to 
Earth's oblateness, and third body effects (gravitational pull from the Sun and Moon).

The model provides ephemeris from an epoch and position specified by a two line element (TLE) input, and can be coded to output orbit position and velocity vectors in the ECI reference frame. The longer this ephemeris runs for without a TLE update, the more orbit position error grows, adversely affecting attitude knowledge. An elaboration on the SGP4 model can be found in Vallado, and an analysis of orbit position knowledge error is conducted by Bowen. ${ }^{1,10}$

\subsection{Reference Vectors}

All body frame sensor readings require their corresponding vectors in a chosen reference frame (orbital in this work) in order to determine spacecraft attitude with respect to the reference frame. Therefore a means was required to look up magnetic field and sun vectors in the orbital reference frame - this will need to be performed onboard the spacecraft whenever the sensors are being read in order to make use of the vectors obtained. Sun direction is determined with code utilizing the equations given in the Astronomical Almanac (see Bowen). ${ }^{1}$ This ephemeris is performed using an input Julian date and outputs the sun direction vector in the ECI frame. The ECI frame vector is then transformed to the orbital frame using $\mathrm{R}$ and $\mathrm{V}$ vectors at the time of interest, obtained from the onboard orbit propagation model. The reference magnetic field vector will likely be obtained by means of a preprogrammed lookup table as developed by Bowen, whose input is the spacecraft position at the time of interest. ${ }^{1}$ However, since this method has not been officially decided upon or programmed in for Exocube, the magfd.m MATLAB function was used instead. This function simulates the Earth's magnetic field using a 
spherical harmonic model, and is described in detail by Bowen. ${ }^{1}$ Inputs to this function are decimal year date and spacecraft position in LLA coordinates (transformed from the orbit propagator ECI outputs), and outputs are magnetic field vector and magnitude at the point of interest in NED coordinates. The NED frame vectors are then transformed to the orbital frame using a series of frame rotations. Figure 10 and Figure 11 below depict a graphical representation of the orbit propagator, sun ephemeris model, and magnetic field model inputs and outputs, as well as the intermediate frame transformations necessary to place the output reference vectors in the desired orbital frame.

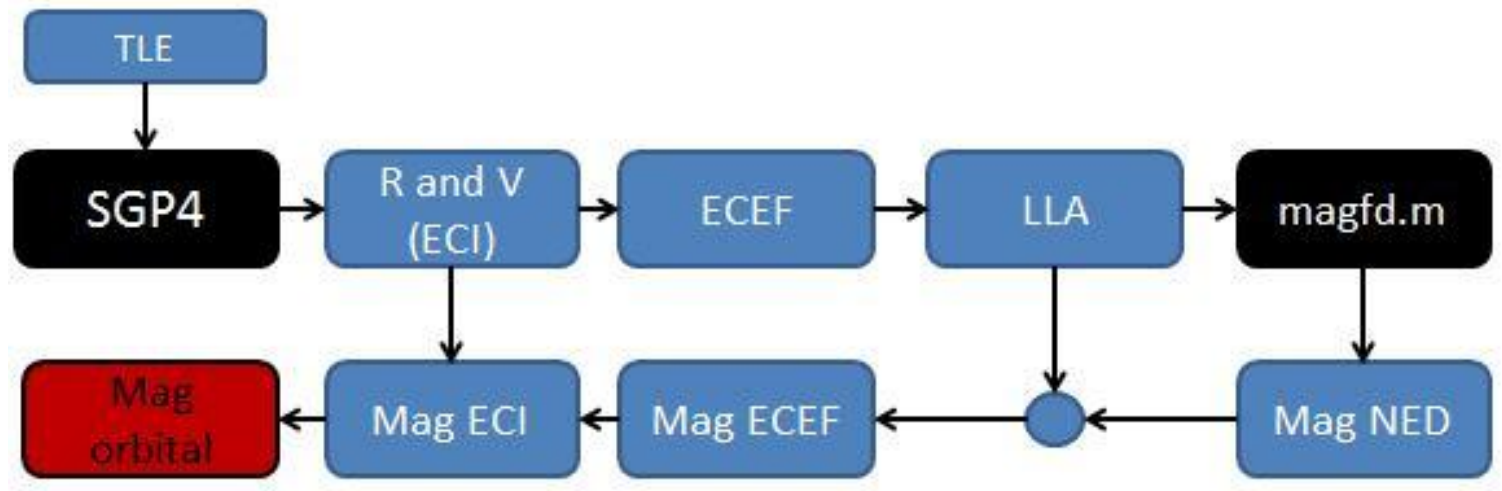

Figure 10. Magnetic field reference vector computation flow.

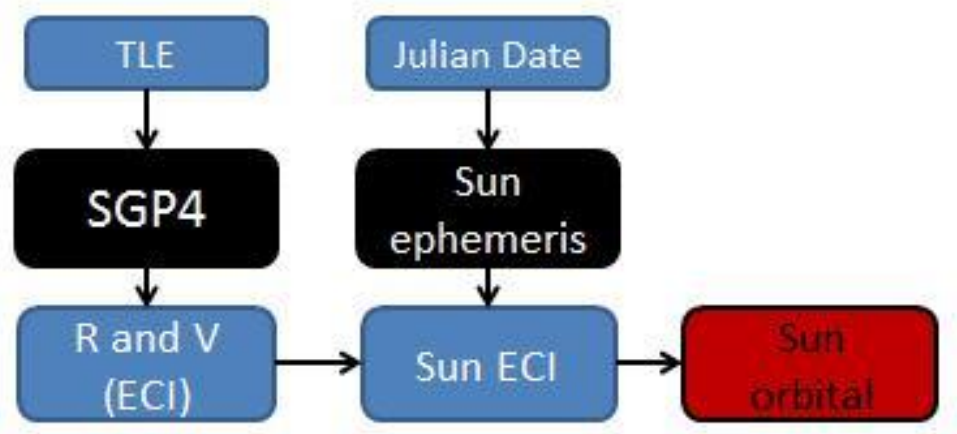

Figure 11. Sun direction reference vector computation flow. 


\section{Attitude Algorithms}

The algorithms presented here have been found through research to be rather common methods of estimating attitude utilizing body vectors read from sensors and corresponding reference vectors computed through the ephemeris models discussed earlier. The following were chosen with the intent of reliably providing Exocube the necessary attitude knowledge with margin for error, while making use of the lower level of sophistication of the attitude hardware types selected.

\subsection{TRIAD}

TRIAD is a very simple deterministic algorithm that is used to compute a rotation matrix (and therefore attitude) between the spacecraft body frame and a reference frame. This is done using two different body frame vectors read from sensors (in this case sun and magnetic field directions) and their corresponding reference vectors. The use of two vector sets to form a rotation matrix overdetermines attitude, so this algorithm indirectly discards some of the vector knowledge by assuming one of these body/reference frame vector pairs is exact, and proceeds to form two triads of orthonormal unit vectors (see Hall for further elaboration). ${ }^{4}$ The following will go through the algorithm process following Hall's explanation using the sun and magnetic field direction sets as applicable to this work. ${ }^{4}$ The equations presented here will assume that the sun direction vector pair is exact, but one can attribute this to either vector pair to start the algorithm.

TRIAD uses a pseudo reference frame of sorts as an intermediary means of forming the final rotation matrix between the body and reference frames (orbital in this work); this frame will be denoted by $t$. It is important to note that all vectors used are normalized 
before being input to this algorithm. To begin, the first base vector set follows the assumption that the sun direction vector pair is exact:

$$
\begin{aligned}
& t_{1 b}=s_{b} \\
& t_{1 o}=s_{o}
\end{aligned}
$$

Here $s_{b}$ and $s_{o}$ denote normalized sun direction vectors in the body and orbital frames, respectively. The second base vector set of the intermediary frame is formed as a unit vector perpendicular to both the sun and magnetic field direction observations with

$$
\begin{aligned}
& t_{2 b}=\frac{s_{b} \times b_{b}}{\left\|s_{b} \times b_{b}\right\|} \\
& t_{2 o}=\frac{s_{o} \times b_{o}}{\left\|s_{o} \times b_{o}\right\|}
\end{aligned}
$$

As with Equation (37), $b_{b}$ and $b_{o}$ are the normalized magnetic field direction vectors in the body and orbital frames. The third and final base vector set is constructed to complete the triad:

$$
\begin{aligned}
& t_{3 b}=t_{1 b} \times t_{2 b} \\
& t_{3 o}=t_{1 o} \times t_{2 o}
\end{aligned}
$$

Two $3 \times 3$ rotation matrices are then created with the placement of the base vectors in columns as

$$
\left[\begin{array}{lll}
t_{1 b} & t_{2 b} & t_{3 b}
\end{array}\right] \text { and }\left[\begin{array}{lll}
t_{1 o} & t_{2 o} & t_{3 o}
\end{array}\right]
$$


The final rotation matrix is then created with

$$
C_{b / o}=C_{b / t} C_{t / o}=\left[\begin{array}{lll}
t_{1 b} & t_{2 b} & t_{3 b}
\end{array}\right]\left[\begin{array}{lll}
t_{1 o} & t_{2 o} & t_{3 o}
\end{array}\right]^{T}
$$

Quaternions giving the orientation of the spacecraft body frame with respect to the orbital reference frame may then be extracted from the rotation matrix $C_{b / o}$ as shown by Curtis. ${ }^{3}$ The equations above are a clear representation of the simplicity of the TRIAD algorithm. However, its use results in increased error due to the assumption that one of the body/reference vector pairs is exact and its subsequent discarding of part of the knowledge given by the other vector pair. Also, in using a sun vector observation, this algorithm will obviously not work in eclipse conditions for this application. TRIAD can still be called on in sunlit conditions to give a somewhat more coarse determination of spacecraft attitude, but it will not suffice for continuous use on board Exocube given its mission requirement for attitude knowledge with the type of sensors anticipated for use.

\subsection{Extended Kalman Filter}

Although very simplistic and easily implemented, the shortcomings of the TRIAD algorithm bring forth the need for a much more robust means of attitude estimation that can be used to satisfy the Exocube mission knowledge requirement continuously in both sunlit and eclipse portions of orbit. An Extended Kalman filter (EKF) was chosen due to its merit in handling noisy data to estimate attitude, as well as the fact that the newer PolySat system boards that will be used to run the implemented software have been assured to be able to handle the increase in computational load. 
A Kalman filter is a recursive algorithm that provides a statistically optimal estimate of a stochastic dynamic state by using a propagation model updated at each iteration with noisy measurements. When the dynamics in question are nonlinear as is the case with the coupled differential equations used to propagate spacecraft attitude, the algorithm is augmented with modifications made to take this nonlinearity into account - the result is an Extended Kalman filter. This paper will not delve into the derivation of the algorithm equations as it is heavily based in the realm of statistics and will not aid the reader much in getting it to work for this application. Rather, the algorithm equations will be presented in an ordered and concise manner, to aid the reader in understanding its purpose and flow. For a more in depth description, analysis, and derivation of this algorithm, see Brown and Hwang, Zarchan and Musoff., ${ }^{2,13}$ The explanations given here are taken largely from Zarchan and Musoff. ${ }^{13}$

\subsubsection{Filter Basis - Dynamics and Measurement Models}

To begin, the nonlinear dynamics of the real world are expressed in state space form as

$$
\dot{x}=f(x)+w
$$

where $x$ is the system state vector, in this case comprised of spacecraft body rates and quaternions such that $x=\left[\begin{array}{lllllll}\omega_{1} & \omega_{2} & \omega_{3} & q_{1} & q_{2} & q_{3} & q_{4}\end{array}\right]^{T} \cdot f(x)$ is a nonlinear function of the state vector, and $w$ is a random process with zero mean, used as a means to account for the somewhat random nature of and/or not fully "known" dynamics that are modeled. The random process is described by the process noise matrix $Q$, which will 
be presented shortly. Moving on, the measurement function, which is also a nonlinear function of the state vector given the means of measurement used, is given by

$$
z=h(x)+v
$$

Here $z$ is the measurement vector, $h(x)$ is a function relating the measurement(s) taken to the state vector (or some portion of it), and $v$ is another zero mean random process - this is described by the measurement noise matrix $R$ which will also be discussed shortly. Equations (42) and (43) provide the models for the nonlinear dynamics and measurement relations that form the basis for the EKF.

\subsubsection{Initialization}

Before the filter algorithm is started, the state vector is initialized with a "best guess" of the actual state to be modeled. Unlike a more basic linear Kalman filter, the EKF assumes that this initial state is close to the actual initial state and filter convergence and performance rely a good deal on this. For this reason, TRIAD is to be used in many cases to help set the quaternion portion of the initial state vector $x_{0}$. Matrices for error covariance, measurement noise, and process noise are set next. The error covariance matrix $P$ can be thought of as providing a prediction of the state estimate error. Because the initial state vector feeding into the EKF is implicitly assumed to have "low" error, the initial error covariance matrix $P_{0}$ has its diagonal elements set "low" and all other elements set to zero. Low is of course a relative term here, and these values are set as a means to tune the filter to what works best for a specific situation. For simulations run in this work, the EKF performed best with regards to convergence time and steady state 
error when these diagonal elements were set between $10^{-3}$ and $10^{-2}$. The process noise matrix $Q$ is initialized in a similar manner, with diagonal elements set to values between $10^{-10}$ and $10^{-7}$ for the simulations performed in this work and all off diagonal elements set to zero. Finally, the measurement noise matrix $R$ is set with its diagonal elements equal to measurement noise variance (standard deviation squared); this depends on the amount of noise anticipated from the sensors and affects the steady state error of the EKF. Off diagonal elements for this matrix are again set to zero. Unlike the error covariance and process noise matrices, the measurement noise matrix is set and not propagated forth or updated within the filter algorithm.

\subsubsection{Algorithm Flow}

As mentioned previously, the EKF is a recursive algorithm. Each iteration is split into two parts: a propagation phase utilizing a hardcoded dynamics model and an update phase utilizing a noisy measurement. These two phases are referred to as a priori and $a$ posteriori respectively, and vectors/matrices in both phases will be denoted with the superscript notation $X^{-}$and $X^{+}$accordingly. The algorithm operates in discrete time, with

a time step $T_{s}$ based on the frequency at which sensor readings are taken (for instance, $T_{S}=0.5 \mathrm{~s}$ corresponds to a sensor read rate of $2 \mathrm{~Hz}$ ).

\subsubsection{A Priori}

The a priori or propagation phase begins by numeric integration of the state using the nonlinear differential equations describing its dynamics. A simple Euler integrator is used with a preset integration time step $(d t)$ determining the number of iterations per filter time step. The integrator time step can be decreased (finer integration), or the integration 
technique itself can be substituted by a more accurate one (like a Runge-Kutta method) for increased accuracy in state propagation, but these would be done at the expense of computational intensity and therefore filter runtime. With the EKF developed in this work, an Euler integrator with $d t=0.1 T_{s}$ gave good performance while remaining very simple and fast.

Following the numeric integration step, the nonlinear dynamics are linearized about the state in the form

$$
F=\frac{\partial f(x)}{\partial x^{+}}
$$

where $F$ is the system dynamics matrix. It is an $n \times n$ Jacobian, $n$ being the number of elements in the state vector. It is important to note here that the linearization is done about the previous a posteriori state, denoted as $x^{+}$, and not the a priori state that was just propagated. If the filter is on its very first iteration, the initial state vector is used instead.

After linearization of system dynamics is calculation of the fundamental matrix $\Phi$. This is formed by the Taylor series expansion of $e^{F T_{s}}$, expressed as

$$
\Phi=I+F T_{s}+\frac{F^{2} T_{s}^{2}}{2 !}+\frac{F^{3} T_{s}^{3}}{3 !}+\cdots
$$

in which $I$ is the identity matrix. An approximation of this series expansion using only the first two terms is much more computationally efficient and will often suffice, thus the fundamental matrix is truncated to 


$$
\Phi \approx I+F T_{S}
$$

The fundamental matrix will be used to propagate the process noise matrix and error covariance matrix in the remaining portion of the a priori phase. Although the EKF is run in discrete time, the process noise matrix has a continuous time update of

$$
Q=\int_{0}^{T_{S}} \Phi(\tau) Q \Phi^{T}(\tau) d t
$$

With a MATLAB implementation as done in this work, this integration can be carried out once and implemented symbolically with appropriate values plugged in at every iteration. One can also forgo this update to simplify things, but at the cost of making the filter a bit less robust. The simulations conducted here however all used a process noise matrix with constant values (unchanging from the initialized matrix) with success.

The final step in the a priori phase of the EKF is propagation of the error covariance matrix. This is carried out as

$$
P^{-}=\Phi P^{+} \Phi^{T}+Q
$$

$P^{+}$simply denotes the error covariance matrix that was updated a posteriori in the previous iteration. In the event that the filter is on its first iteration, the initial error covariance matrix $P_{0}$ is used instead. This completes the a priori portion of the EKF.

\subsubsection{A Posteriori}

The a posteriori, or measurement update phase of the EKF begins with formation of the simulated measurement vector $h$. This is set as 


$$
h=\left[C b_{o}\right] \quad \text { or } \quad h=\left[\begin{array}{l}
C b_{o} \\
C s_{o}
\end{array}\right]
$$

where $C$ is the quaternion DCM from Equation (11) and $b_{o}$ and $s_{o}$ are $3 \times 1$ orbital frame magnetic field and sun direction reference vectors (these vectors are normalized before being used here since vector length does not represent orientation). The DCM is calculated using the quaternions generated in the state propagation phase, and the reference vectors are fed in from the orbit propagator and reference vector lookup methods discussed previously in Chapter IV. Attitude . During eclipse, $h$ is a $3 \times 1$ vector formed only by the magnetic field reference vector lookup, and in sunlit conditions it is augmented to the second 6x1 vector presented by Equation (49) (since sunlit portions of an orbit permit use of a sun sensor as well).

The measurement sensitivity matrix $H$ is set next. Similar in its formulation to the system dynamics matrix $F$, this is a linearization of the nonlinear measurement function about the current state vector, generated in the propagation phase of the filter algorithm. It is expressed as

$$
H=\frac{\partial h(x)}{\partial x^{-}}
$$

$H$ is a Jacobian matrix of size $m \times n, m$ being the number of elements in the measurement matrix and $n$ the number of elements in the state vector. Therefore the size of $H$ is dependent on the sensor(s) used at the time of interest. In this case, $H$ is a $3 \times 7$ matrix if only the magnetic field measurement is used (eclipse condition), and 6x7 when both measurements are used. 
Following formation of the measurement sensitivity matrix is calculation of the Kalman gain. This works mainly as a weighing factor determining the effect that the read in body measurement has on the a posteriori state update; this will become more apparent when this update is presented shortly. The Kalman gain is computed using the previously propagated error covariance matrix, measurement sensitivity matrix, and preset measurement matrix as shown by

$$
K=P^{-} H^{T}\left(H P^{-} H^{T}+R\right)^{-1}
$$

Of course the size of the measurement matrix $R$ is dictated by the size of the measurement vector; it is a diagonal $n \times n$ matrix as described in Section 5.2.2 Initialization, $n$ being the number of elements of the measurement vector in use.

The state vector is updated next. This is done by adding the a priori propagated state vector $x^{-}$to a weighted difference between the body frame sensor measurement vector $z$ and the corresponding calculated measurement vector $h$ as seen below

$$
x^{+}=x^{-}+K(z-h)
$$

In this work the row vector $z$ is comprised of body frame magnetic field and/or sun direction vectors (these vectors are also normalized for the same reason as done for the vectors used in the calculation of $h$ in Equation (49)). The difference $(z-h)$ is referred to as the measurement residual, and can be thought of as the difference between the actual measurement read from the sensors and the estimated measurement. Again, the Kalman gain is used to weigh this residual based on how much the algorithm "believes" the measurement to be true over its own propagation phase. A larger Kalman gain 
therefore means that the sensor measurement taken at that particular iteration has more of an effect on the a posteriori state update.

The final step in the a posteriori phase of the EKF is an update of the error covariance matrix, given by

$$
P^{+}=(I-K H) P^{-}(I-K H)^{T}+K R K^{T}
$$

More compact or expanded forms of this equation exist (see Brown and Hwang, Zarchan and Musoff), but this form was settled on and utilized here since it provided good filter performance in the simulations conducted. ${ }^{2,13}$ The end of this measurement update phase concludes one full iteration of the EKF - all vectors and matrices are carried into the next propagation phase and the same process starts over until the algorithm is terminated. The state vector calculated in each iteration can be used to estimate spacecraft attitude here, since a portion of it consists of quaternions relating the body frame to the orbital reference frame (body rates are also estimated since they are included in the state vector).

\subsubsection{Overview}

To reiterate, the Extended Kalman Filter (EKF) is a recursive algorithm running in discrete time that will be used in this work to give a steady state estimate of spacecraft body attitude with respect to the orbital reference frame. This is done utilizing a hardcoded dynamic model augmented by noisy measurements, and will ideally converge to a steady state error lower than that present in the sensors read. This convergence relies on a wide array of variables including initial conditions, filter propagation accuracy, sensor measurements used, and the very nature of the dynamic environment modeled and 
measured. Specific factors affecting convergence and steady state error in this work will be presented further on with simulation results. As a final note in this section, a simple graphical representation of this algorithm is shown in Figure 12.

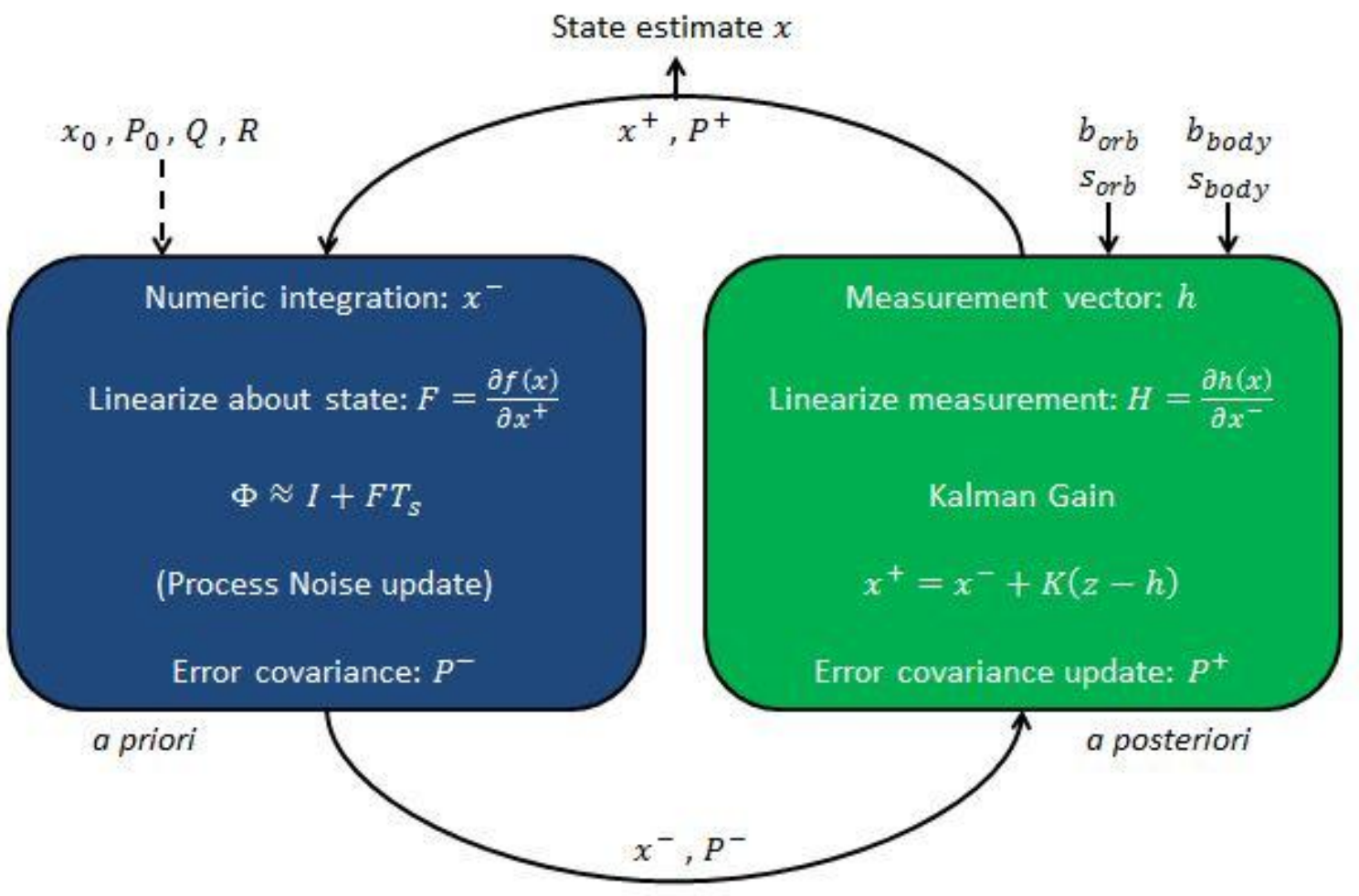

Figure 12. Graphical representation of Extended Kalman Filter. 


\section{Simulation}

Numerous simulations were conducted in this work with the intent of proving the feasibility of providing attitude estimation of Exocube to within the \pm 5 degree ram knowledge requirement with the sensor types to be used and the algorithms presented in the previous chapter. Simulations were conducted exclusively in MATLAB with the exception of one simulation type using vectors fed in from STK as a means of algorithm verification.

The built in MATLAB function ode45 was used to propagate spacecraft attitude in all simulations, solving the differential equations presented in Chapter III. Spacecraft Rotational Equations of Motion describing rotational kinematics and dynamics. All environmental factors and satellite hardware considered in those equations were modeled (orbit eccentricity, gravity gradient torque, momentum wheel, etc.). Orbit ephemeris methods varied and will be discussed in their respective sections to come. Both attitude propagation and orbit ephemeris were conducted with a consistent time step in simulation, eliminating the need for any form of interpolation to match corresponding vectors for specific points in time.

Before any simulations were run, satellite hardware and software process errors that would affect steady state attitude estimation needed to be defined. The most apparent sources of error were of course those inherent in the solar angle sensor and magnetometer and the known precision of their desired locations and alignments on the spacecraft body. Errors due to functions and processes onboard Exocube that would affect attitude estimation would stem from the orbit propagator, reference vector ephemeris/lookups, 
real time clock, and sensor calibration routines, to name a few. Sensor error, orbit propagation error, and magnetic field and sun direction reference errors were those chosen to be accounted for in the simulations run, as they are anticipated to be the most influential on attitude estimation. The level of error attributed to these has not yet been characterized, so these errors were all set higher than anticipated (based on sensor spec sheets and previous thesis work) as a means of adding margin and accounting for unmodeled sources of error.

\subsection{Modeling Error}

The aforementioned sources of error were set in simulation by using the MATLAB function randn, which generates a user selected $m \times n$ vector/matrix of random elements with standard deviation $\sigma$. This vector of random numbers is added to sensor derived body measurement vectors and reference vectors at every iteration in simulation as such, using magnetic field as an example:

$$
\begin{aligned}
& \tilde{b}_{b}=b_{b}+\sigma \cdot \operatorname{randn}(3,1) \\
& \tilde{b}_{o}=b_{o}+\sigma \cdot \operatorname{randn}(3,1)
\end{aligned}
$$

Here $\tilde{b}_{b}$ and $\tilde{b}_{o}$ are the body frame and orbital reference frame magnetic field direction vectors with error added. Error added to the body frame vector reflects that from sensor measurement, and error added to the orbital frame vector accounts for the magnetic field lookup and orbit propagator (since the former is dependent on the latter). The same convention is followed for sun direction vectors. To make better sense of the error summed onto these vectors, angular error needed to be related to the standard deviation 
of random noise additions implemented. This was done using the dot product rule between each vector generated and its corresponding error corrupted counterpart. An average angular error was then determined from a given standard deviation input using a large sample size ( $>5000)$ of generated vectors, each of which calls on the randn function separately (this ensures that the standard deviation input is held constant but the exact same random number set isn't generated for each vector). The vectors input and generated as shown in Equation (54) were manipulated to derive the angle between them with

$$
\theta_{\text {dev }}=\cos ^{-1}\left(\frac{b_{o} \cdot \tilde{b}_{o}}{\left\|b_{o}\right\|\left\|\tilde{b}_{o}\right\|}\right)
$$

where $\theta_{d e v}$ is the angular deviation between the input vector and its corresponding vector with the random noise addition (orbital frame magnetic field direction used as an example). Equation (55) is applied to a large sample size of vectors as mentioned previously, for instance to all of the orbital frame reference vectors generated in an orbit with an ephemeris step time of one second. The resulting angular deviations are recorded and the mean of the entire sample is calculated, thus generating an average angular error given a standard deviation random noise addition to a measurement or reference vector. 
This characterization is depicted by the generated plot below.

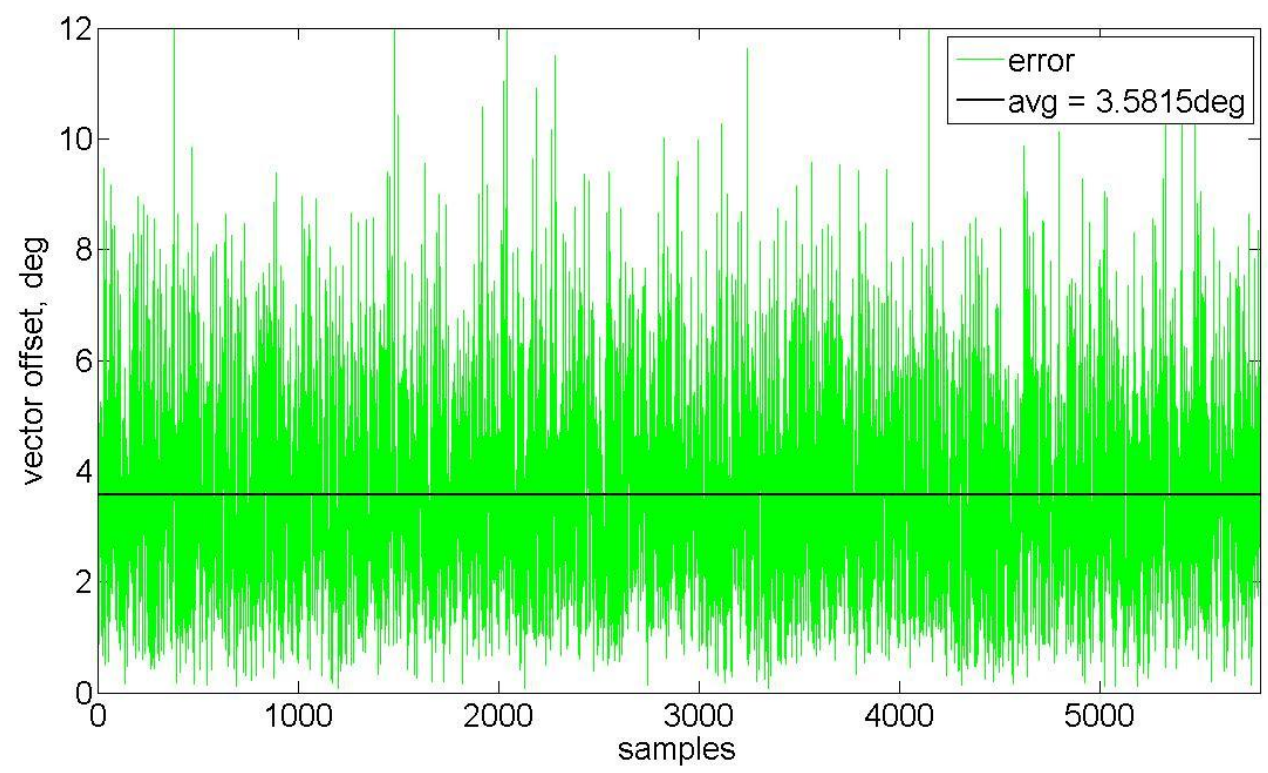

Figure 13. Angular deviation between vectors as described with $\sigma=0.05$.

Figure 13 shows that using Equations (54) and (55), random noise with standard deviation of 0.05 added onto vectors results in an average angular deviation of 3.58 degrees. This mode of characterization between input standard deviation random noise and angular deviation is utilized in this work to model the desired levels of angular error attributed to any measurement and reference vectors. The table below gives this same characterization of average angular error for a range of standard deviation inputs. 
Table 1. Average angular error resulting from varying standard deviation random noise inputs.

\begin{tabular}{|c|c|}
\hline Standard deviation random noise input & Average angular error (degrees) \\
\hline 0.015 & 1.07 \\
\hline 0.03 & 2.13 \\
\hline 0.05 & 3.58 \\
\hline 0.07 & 5.11 \\
\hline 0.09 & 6.45 \\
\hline 0.11 & 7.83 \\
\hline 0.13 & 9.44 \\
\hline 0.15 & 10.8 \\
\hline 0.17 & 12.2 \\
\hline
\end{tabular}

\subsection{MATLAB Simulation}

As mentioned previously, nearly all simulations conducted for this thesis were run purely in MATLAB. This was done to allow efficient editing of simulation input parameters, allowing different scenarios to be run rapidly and ultimately helping "tune" and characterize the performance of the attitude estimation algorithms used - namely the EKF. Below is a graphical representation of the MATLAB simulation flow. 


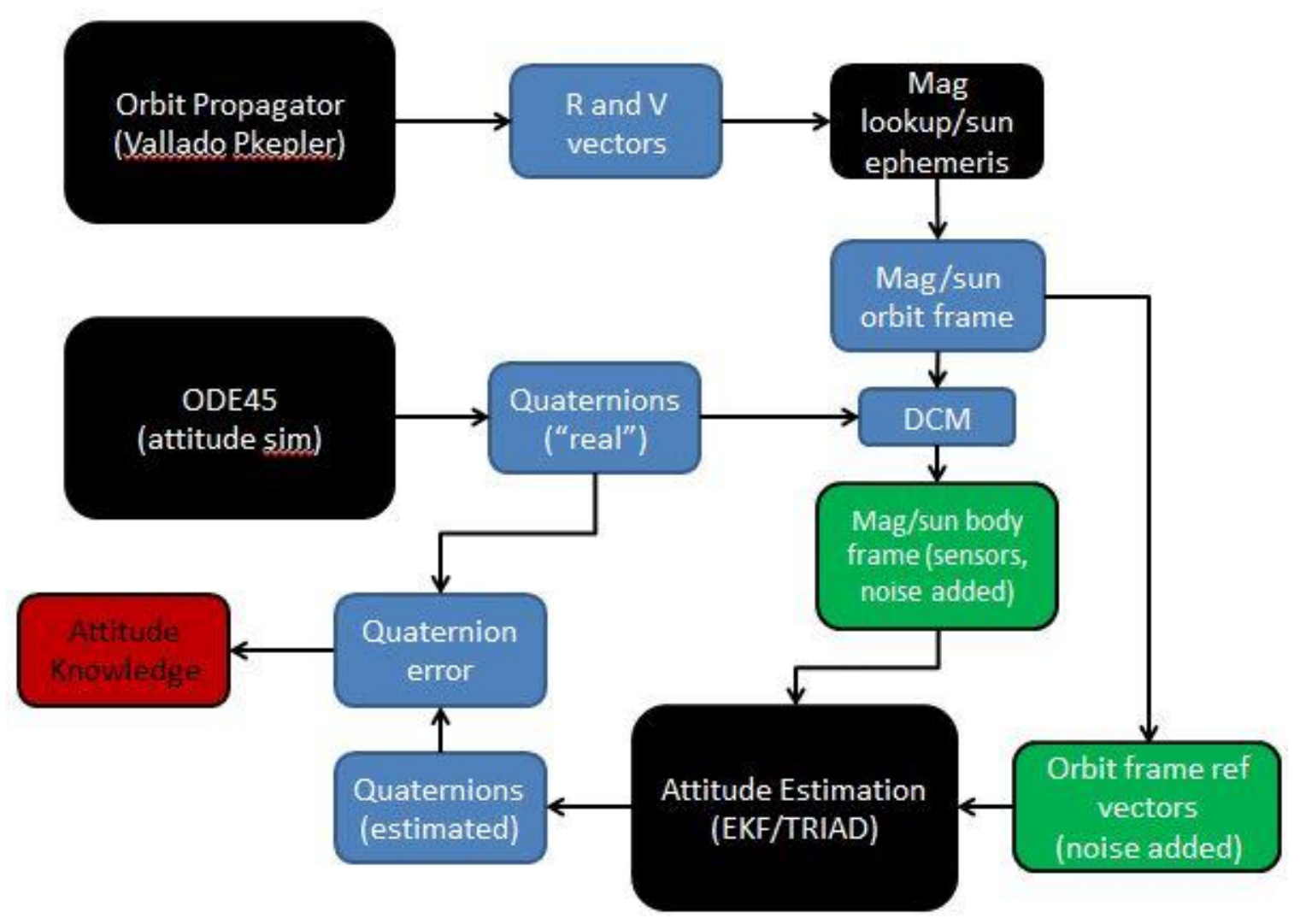

Figure 14. MATLAB simulation flowchart.

The four black boxes represent the main functions performed in simulation: orbit propagation, attitude simulation (kinematics and dynamics), magnetic field lookup and sun vector ephemeris, and attitude algorithms. The main script sets a master step time for all of these functions to ensure that all generated vectors remain matched in time without the need for interpolation.

The orbit propagator utilizes the PKepler algorithm and all necessary subfunctions as found in Vallado; this was set to only account for the $\mathrm{J} 2$ perturbation effect on argument of perigee and right ascension of ascending node (RAAN). ${ }^{10}$ Inputs are radius of perigee and apogee $(\mathrm{km})$, initial true anomaly (degrees), RAAN (degrees), argument of perigee 
(degrees), inclination (degrees), the number of orbits to be propagated, and the propagation time step (master time step is used in seconds).

The magnetic field vector lookup and sun ephemeris model functions use the orbit radius and velocity vectors generated by the orbit propagator to find the desired magnetic field and sun direction orbital reference frame vectors at each time step. This is accomplished as described in Chapter IV. Attitude, keeping the epoch date consistent between the magnetic field and sun ephemeris models (magfd.m requires a decimal year input, while the sun ephemeris requires Julian date).

Following the generation of reference vectors, spacecraft attitude is simulated for the same number of orbits with the same master time step. This is done using ode 45 and the kinematic and dynamic equations describing spacecraft rotational motion from Chapter III. Spacecraft Rotational Equations of Motion, as stated earlier. For the equations in use, defined spacecraft parameters include body inertia tensor $\left(\mathrm{kgm}^{2}\right)$, momentum wheel inertia $\left(\mathrm{kgm}^{2}\right)$ and angular rate $(\mathrm{rad} / \mathrm{s})$, spherical damper inertia $\left(\mathrm{kgm}^{2}\right)$ and viscous damping coefficient $(\mathrm{Ns} / \mathrm{m})$, spacecraft maximum ram facing area $\left(\mathrm{m}^{2}\right)$, and atmospheric densities at perigee and apogee $\left(\mathrm{kg} / \mathrm{m}^{3}\right.$, taken from Wertz). ${ }^{11}$ The initial state vector consisted of eleven elements, given by

$$
x=\left[\begin{array}{lllllllllll}
\omega_{1} & \omega_{2} & \omega_{3} & q_{1} & q_{2} & q_{3} & q_{4} & \omega_{1_{d}} & \omega_{2_{d}} & \omega_{3_{d}} & \theta
\end{array}\right]^{T}
$$

The first seven elements are spacecraft body rates and quaternions, $\omega_{1_{d}} \omega_{2_{d}}$ and $\omega_{3_{d}}$ are spherical damper rates as presented in Section 3.2.3 Spherical Damper, and $\theta$ is orbit true anomaly, propagated at every time step to account for the effect of orbit eccentricity on 
the gravity gradient moment modeled. Values input to this state vector were varied depending on the mission scenario simulated, and will be presented with each type. Attitude simulation outputs utilized are spacecraft body rates and quaternions.

An intermediary step in simulation, quaternions output from the ode45 attitude simulation are used to transform the previously generated orbital frame reference vectors into the spacecraft body frame by means of the DCM in Equation (11). Random noise is added to the body frame vectors at each time step as described in the previous section, modeling a desired amount of sensor error and readying then for use by the attitude algorithms as "real world" body frame measurements. The orbital frame reference vectors are called on once more to be used as reference vectors for the attitude algorithms. Again, random noise is added to account for errors present in orbit propagation and magnetic field and sun ephemeris models.

Attitude algorithms were run next, utilizing the simulated body frame sensor measurement and orbital reference frame vectors. The EKF is used predominantly within this block, while the use of TRIAD is limited to generating a quaternion set for the EKF initial state vector. The EKF is run as explained in Chapter V. Attitude Algorithms, using the master time step as its read rate and appropriate measurement and reference vectors at each iteration. As a basic means of simulating eclipse conditions inevitably encountered in every orbit in LEO, the filter switches between a magnetometer only measurement and a magnetometer/sun sensor measurement every half orbit. This aids in demonstrating the ability of the algorithm to sharply transition between measurement types. The state vector utilized by the EKF is just seven elements comprised of spacecraft body rates and 
quaternions. To keep the state vector from becoming overcomplicated and to lessen computational load, the dynamics modeled within the EKF algorithm only consider spacecraft kinematics and dynamics with a gravity gradient moment in a circular orbit and a constant rate momentum wheel (if used). This was deemed a valid means of state propagation due to the fact that the anticipated orbit for Exocube will be of very low eccentricity ( 0.01 order of magnitude) and that the other moments modeled in the "real world" ode45 simulation - spherical damper and drag - are lower in magnitude than the gravity gradient moment (see Sellers regarding a comparison of drag and gravity gradient moments for Exocube). ${ }^{8}$ To reiterate previous explanation, initialization of this algorithm sets the diagonal elements of the error covariance matrix $P$ between $10^{-3}$ and $10^{-2}$, diagonal elements of the process noise matrix $Q$ between $10^{-10}$ and $10^{-7}$, and diagonal elements of the measurement noise matrix $R$ to the square of the standard deviation of random noise input to the corresponding measurement vectors fed in. An initial state $x_{0}$ is also set, with or without the use of TRIAD to set its quaternions. As done with the ode45 simulations, the initial state vector used here varied depending on the dynamic state of the spacecraft modeled. Initial state vectors will be presented on a case by case basis with the results to come.

Outputs of the EKF (and therefore the attitude estimation simulation block) are estimates for spacecraft body rates and quaternions for every time step. The estimated quaternions are compared against those generated by the ode 45 simulation to give an end result for attitude knowledge. This is done as explained in Section 2.9 Quaternions of this work, in which the quaternion error is found between the "actual" from ode 45 and the estimated 
from the EKF. Angles are then extracted from these describing the magnitude of total angular deviation about the roll, pitch, and yaw axes. This work is concerned with the roll axis total angular deviation, since the mission requirement for Exocube dictates \pm 5 degrees of knowledge about the instrument aperture vector which correlates to the body roll axis.

\subsection{MATLAB/STK Simulation}

Simulations conducted purely in MATLAB were very useful in characterizing the capabilities of the attitude algorithms researched and employed in this work, and allowed for simulation input parameters to be changed rapidly, allowing many different scenario types to be run efficiently. Those simulations were lacking in a means of verification, however, and for that reason orbit ephemeris generated by STK was utilized to augment the MATLAB simulation for attitude dynamics and attitude estimation. Verification was provided not only by the use of an external, professional source for simulation, but also by using the new means of orbit simulation to structure the simulation flow more closely to a real world scenario for Exocube. The resulting structure for this particular type of simulation is shown below. 


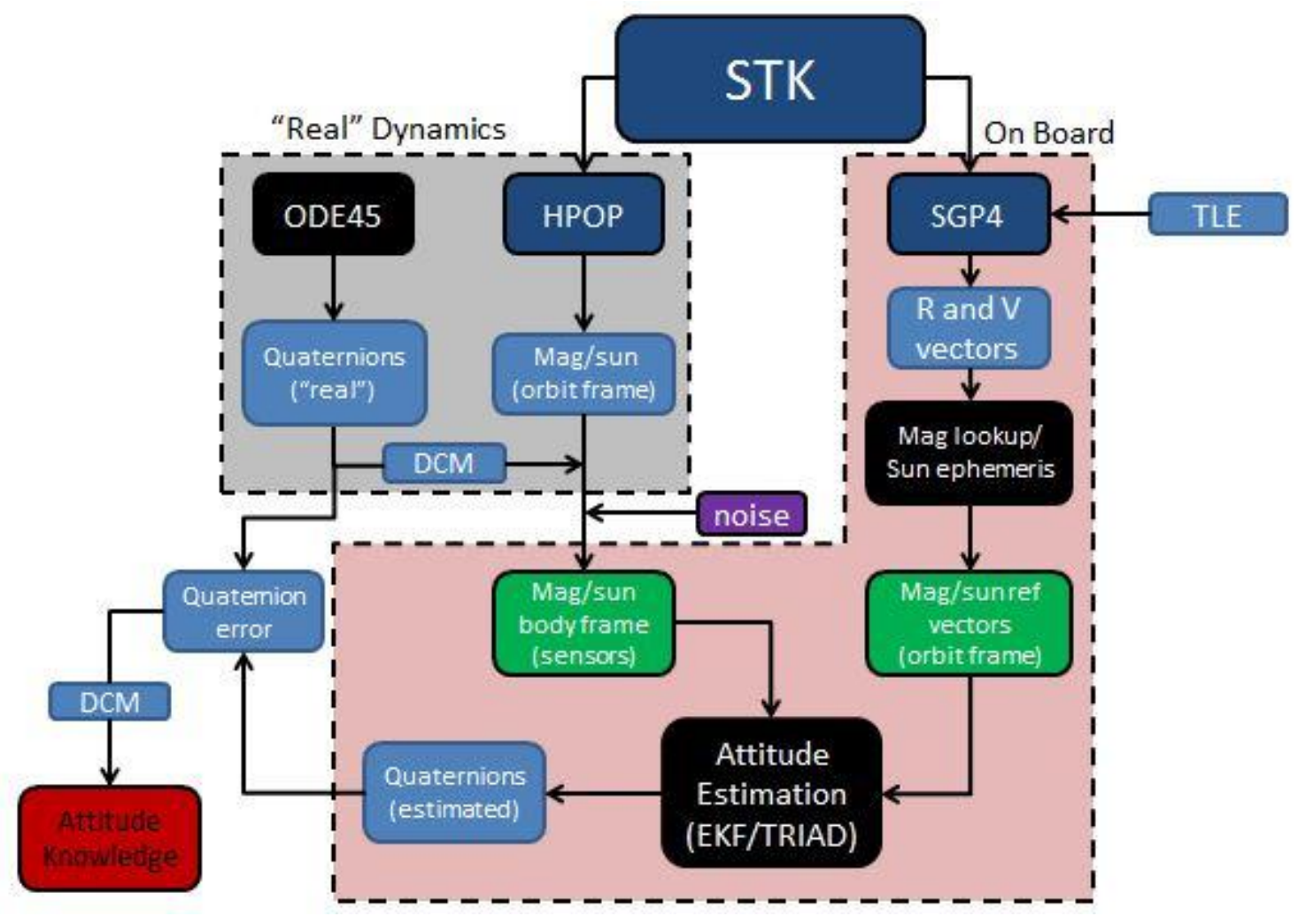

Figure 15. MATLAB/STK simulation flow.

Although at first glance this method appears more complicated than the pure MATLAB implementation, it is carried out in the same manner with the exception of the means used to generate the body frame sensor measurement vectors and corresponding orbital frame reference vectors.

To begin, two different orbit propagators are used in STK to generate ephemeris for the same initial conditions and satellite properties. These are the High Precision Orbit Propagator (HPOP), and Simplified General Perturbations model (SGP4). HPOP is the more accurate of the two, providing in its propagation scheme higher fidelity modeling of effects from Earth's gravitational field, atmospheric drag, solar radiation pressure, Earth 
albedo and third-body gravitational effects. It is initialized with an orbit epoch date/time and standard orbit parameters such as semimajor axis, eccentricity, inclination, RAAN, argument of perigee, etc. As mentioned in Chapter IV. Attitude, SGP4 computes ephemeris from an epoch dictated by a TLE, therefore an initial TLE is input to match the initial orbit parameters specified for HPOP. Both propagators generate ephemeris for the same length of time using the same time step in order to have all vectors matched in time as done with all simulations in this work.

HPOP is used to simulate a "real world" environment from which body frame measurements will be formed, while the SGP4 propagator is used to generate R and V vectors for computing the corresponding orbital frame reference vectors, as will be done onboard Exocube. The original intention was to use the same flight code for SGP4 ephemeris, but because that has yet to be completed for the satellite, SGP4 was employed via STK instead. Future simulations should utilize flight code for a more accurate means of determining attitude algorithm performance.

As shown in Figure 15, the orbital frame reference vectors generated by HPOP are rotated into the body frame using the quaternion outputs of the ode 45 kinematics/dynamics simulation at each corresponding time step. This is done in exactly the same manner as conducted for the previously presented MATLAB simulation. The body frame vectors all have random noise added on, resulting in a "real world" model of noisy in situ sensor measurements. SGP4 is fed a TLE for initialization, and the R and V vectors generated are fed into the magnetic field lookup and sun ephemeris models. Because SGP4 is of lower accuracy than HPOP, orbit position error is inherent in the 
calculated orbital frame reference vectors. Additional error (albeit a small amount) is added onto the magnetic field and sun direction reference vectors to account for errors attributed to their lookup/ephemeris models (0.088 degrees and 0.01 degrees respectively, based off of Bowen's findings). ${ }^{1}$

Having modeled body frame sensor measurement and orbital frame reference vectors, the remainder of the simulation is run in the exact manner as conducted in the previous section. One final note regarding this method of simulation is that the SGP4 algorithm is fed only one TLE at its start; no additional TLEs are fed in and its error in orbit propagation with respect to HPOP grows accordingly. Future work will need to determine how often TLEs will need to be uploaded to Exocube in order to maintain steady state attitude knowledge to within the mission specific requirement. 


\section{Results}

The following will go through each type of scenario run in the simulations described in the previous chapter. Spacecraft dynamics and attitude estimation results will be presented and explained concisely in a case by case manner, with driving simulation parameters stated for each case conducted.

\subsection{MATLAB Simulation}

Simulations conducted purely in MATLAB are the most prevalent in this work and have been used to characterize the necessary means for and performance of the attitude estimation algorithms (namely the EKF) employed. This is done for primarily for the "science mode" phase of the mission, in which Exocube is oriented to within the control requirements and the instrument payload is taking data, thus levying the attitude knowledge requirement. Several other mission phases, each of which describe different dynamic states that Exocube is anticipated to be in throughout mission life, were also simulated to determine how well the EKF could potentially perform under varying circumstances. All simulations presented in this section were conducted with the current anticipated orbit for Exocube of 400x670 km at $98^{\circ}$ inclination. Values for argument of perigee, RAAN, and initial true anomaly were all set to zero. To reiterate Section 2.10 Attitude Representations, all results for attitude knowledge are presented as total angular deviation about the roll axis, and all results for simulated spacecraft dynamics are presented using the standard yaw-pitch-roll angles. 


\subsubsection{Science Mode}

Attitude estimation for Exocube in this this mission phase is the central focus of this thesis, since the mission requirement for attitude knowledge is enforced for times at which the payload Exos is taking data. In this simulation scenario the ode45 initial state vector elements are driven by mission requirements regarding attitude control, which once again state that Exocube shall be controlled to within $\pm 10^{\circ}$ of its nominal orientation and that its body rates shall be at most $0.1 \%$ about all three axes. In dynamics simulations it was found that a $0.1 \% \mathrm{~s}$ rate disturbance causes oscillations outside of the $\pm 10^{\circ}$ angular bounds, even with the gravity gradient booms deployed and momentum wheel operating. This thesis is not at all concerned whether or not this was realized at the time the requirements were set, so the rate disturbance applied is simply set to half of the control limit to keep steady state oscillations very close to or within $\pm 10^{\circ}$. A $5^{\circ}$ offset was also applied to each angle, which set the ode 45 initial state vector to $x=[0.00085,0.00085,0.00085,0.042,0.045,0.042,0.997,0,0,0,0]^{T}$ (body rates are in rad/s). This initialization was found to give a worst case oscillation that was still within the control requirements, and as such was used to determine EKF performance for this scenario. The momentum wheel is on, and gravity gradient booms are deployed, increasing the spacecraft inertia tensor. Spacecraft attitude dynamics are shown below using the initial disturbances and offsets discussed for the momentum wheel at $500 \mathrm{rpm}$ and then at $1500 \mathrm{rpm}$. 


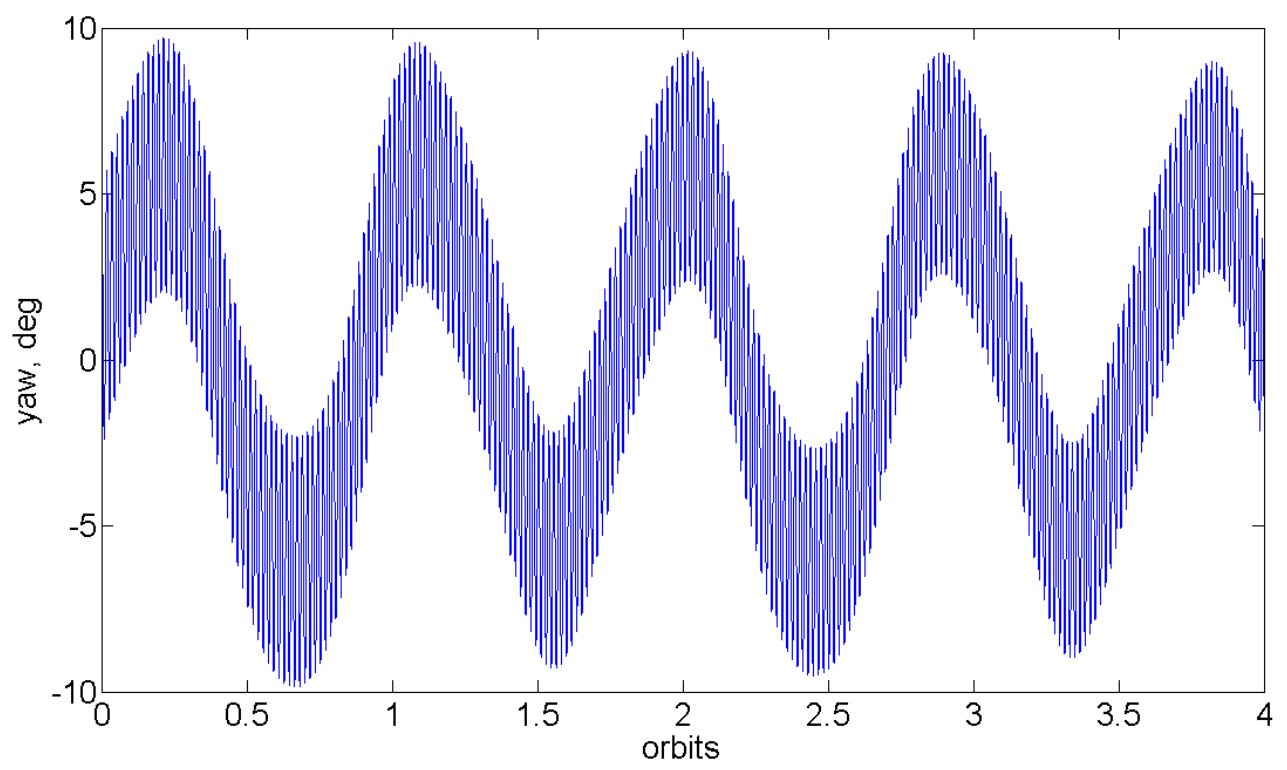

Figure 16. Science mode dynamics, momentum wheel at 500 rpm, yaw axis (b1).

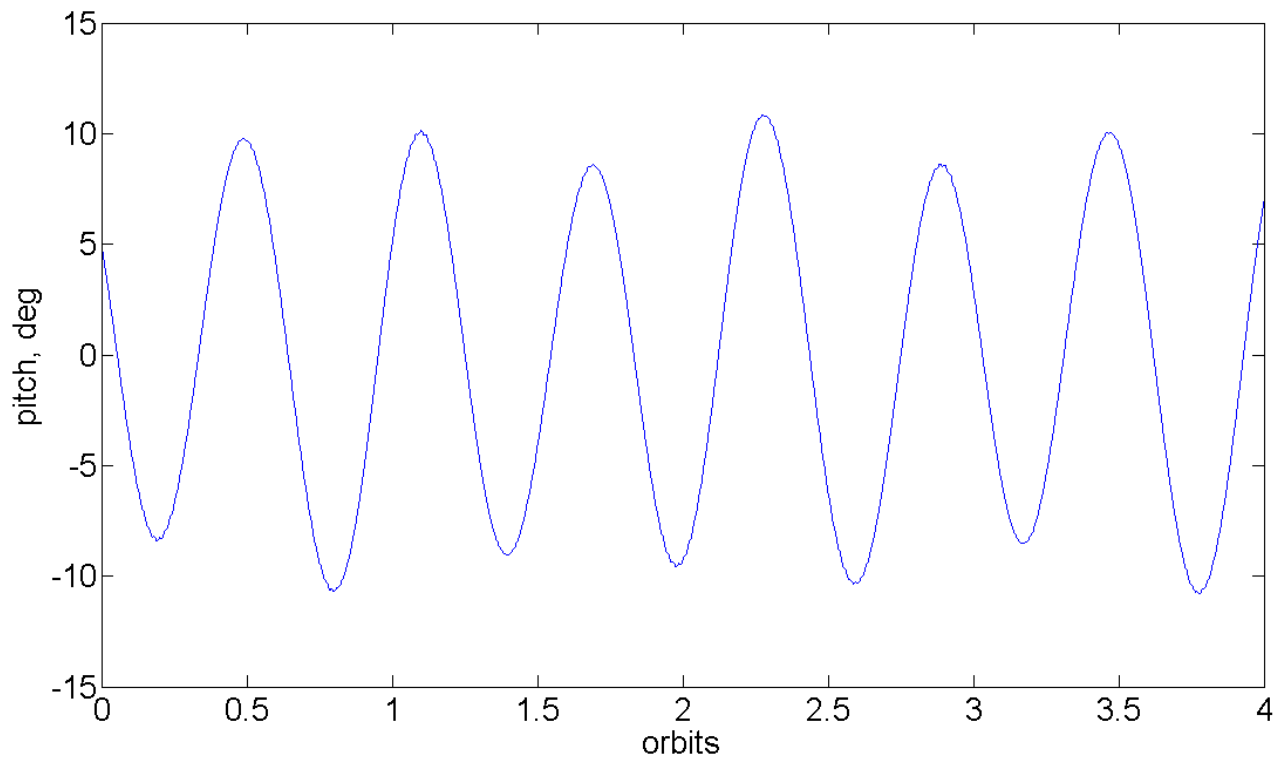

Figure 17. Science mode dynamics, momentum wheel at $500 \mathrm{rpm}$, pitch axis (b3). 


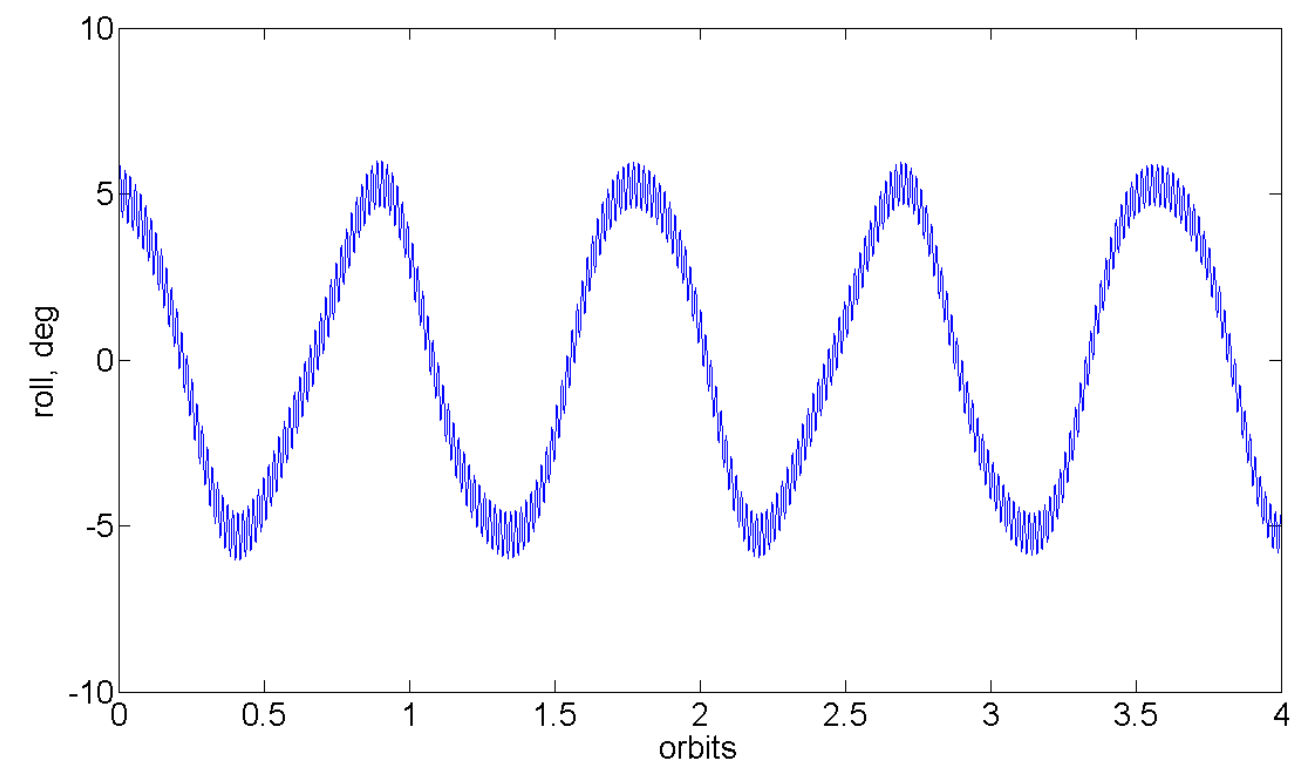

Figure 18. Science mode dynamics, momentum wheel at $500 \mathrm{rpm}$, roll axis (b2).

The secondary frequency seen embedded in the coupled yaw and roll axes oscillations is jitter induced by rate disturbances applied with the use of the momentum wheel, as mentioned in Section 3.2.2 Momentum Wheel - Gyrostat Equations. The momentum wheel also drives overall oscillation amplitude of these two axes noticeably lower than the pitch axis on which it is mounted to in the dynamics model. Wheel rate was increased to $1500 \mathrm{rpm}$ for the plots shown below. 


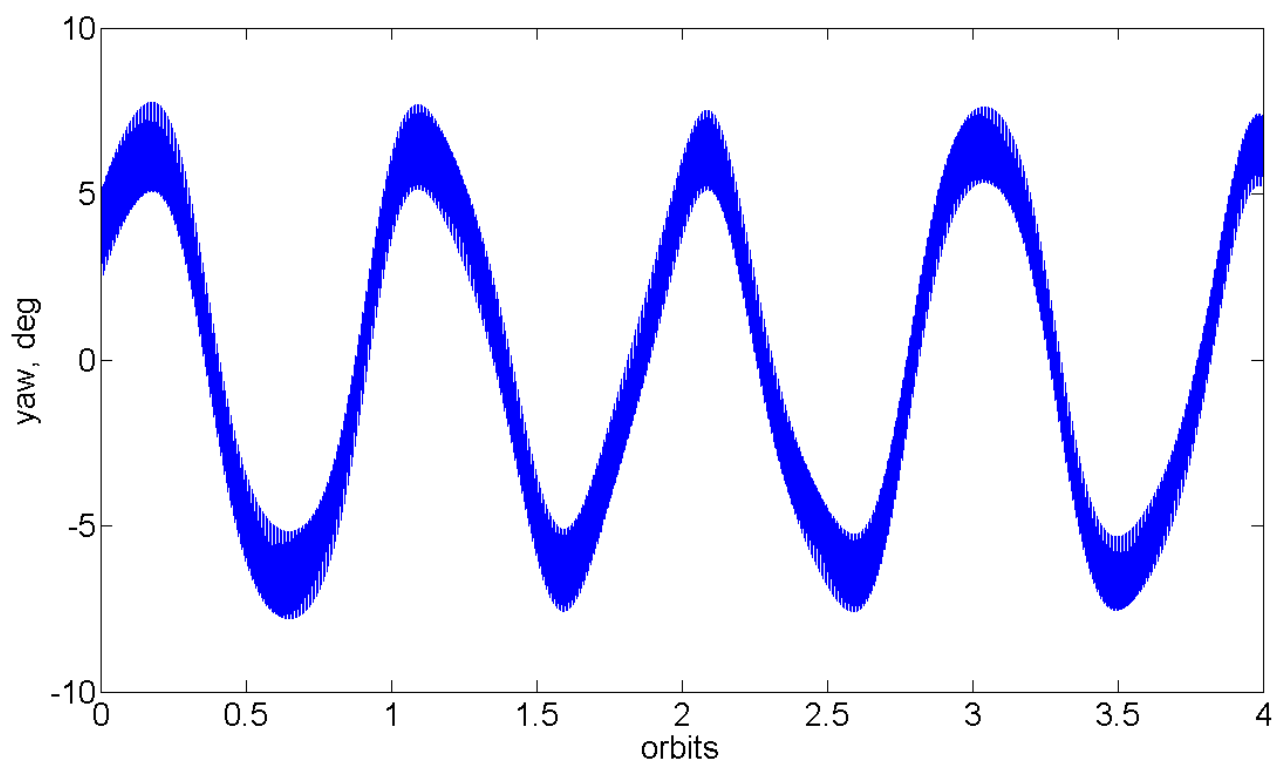

Figure 19. Science mode dynamics, momentum wheel at 1500 rpm, yaw axis (b1).

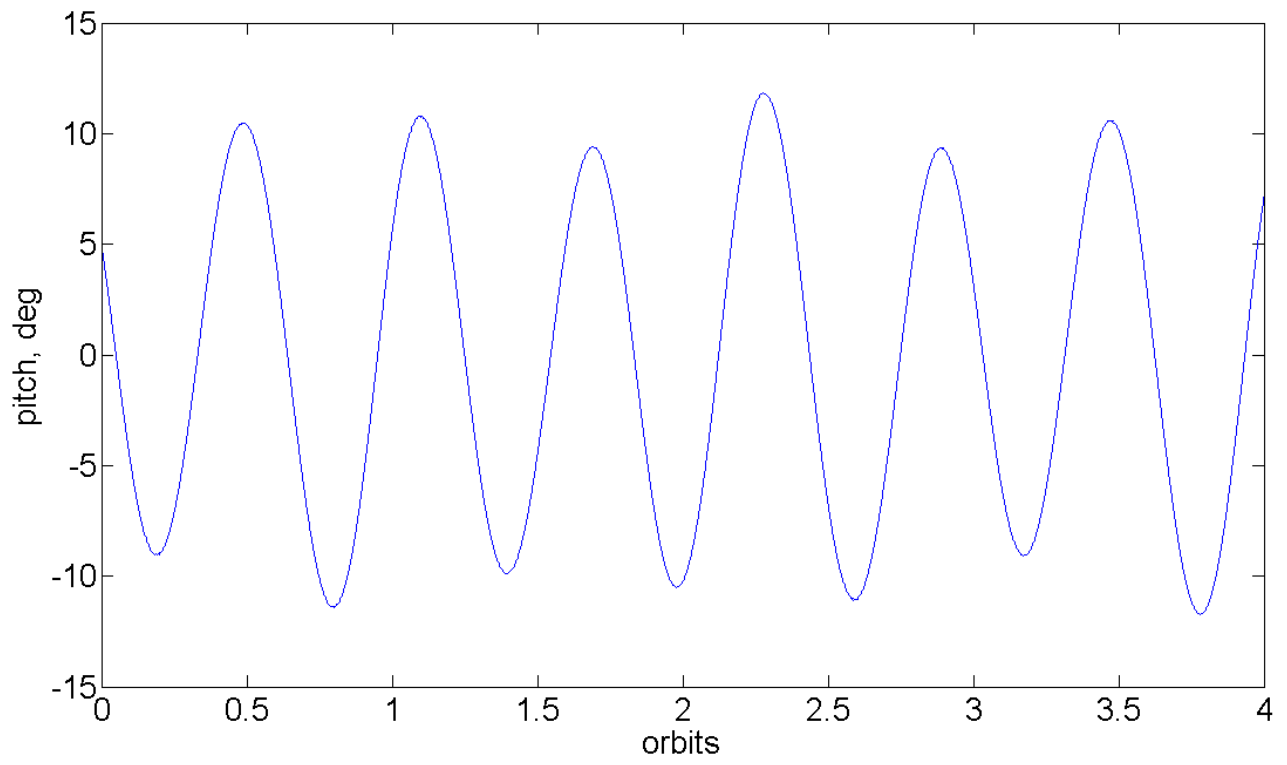

Figure 20. Science mode dynamics, momentum wheel at $1500 \mathrm{rpm}$, pitch axis (b3). 


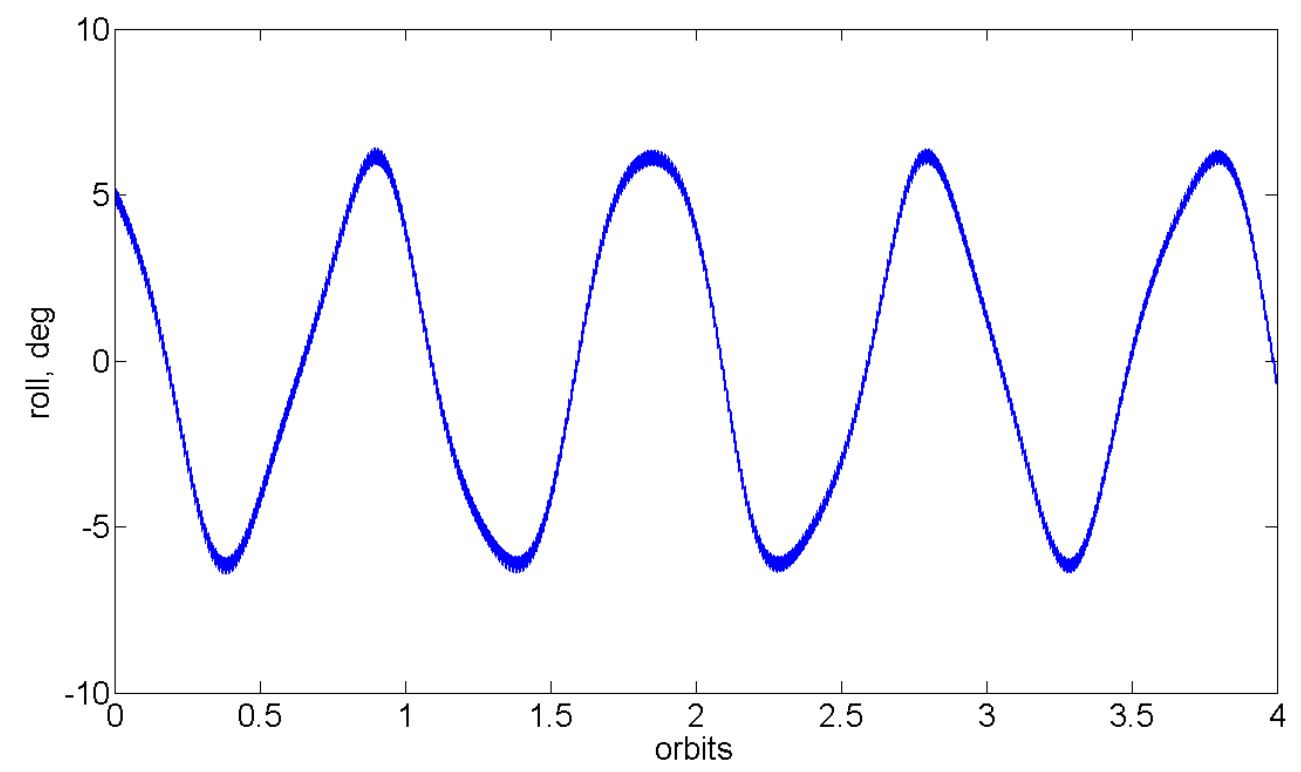

Figure 21. Science mode dynamics, momentum wheel at $1500 \mathrm{rpm}$, roll axis (b2).

The higher rotational rate of the pitch axis momentum wheel provides tighter control on the yaw and roll axes, yet the increased jitter frequency will require a higher EKF sample rate to reliably operate. Attitude estimation simulations will now be discussed for the two wheel rates presented.

Case 7.1.1.1: Science Mode, momentum wheel at $500 \mathrm{rpm}$, magnetometer only, $2 \mathrm{~Hz}$ sample rate

EKF initial state: $x_{0}=[0.002,0.002,0.002,0,0,0,1]^{T}$. Body rates set slightly higher than control requirement threshold, nominal orientation assumed. 


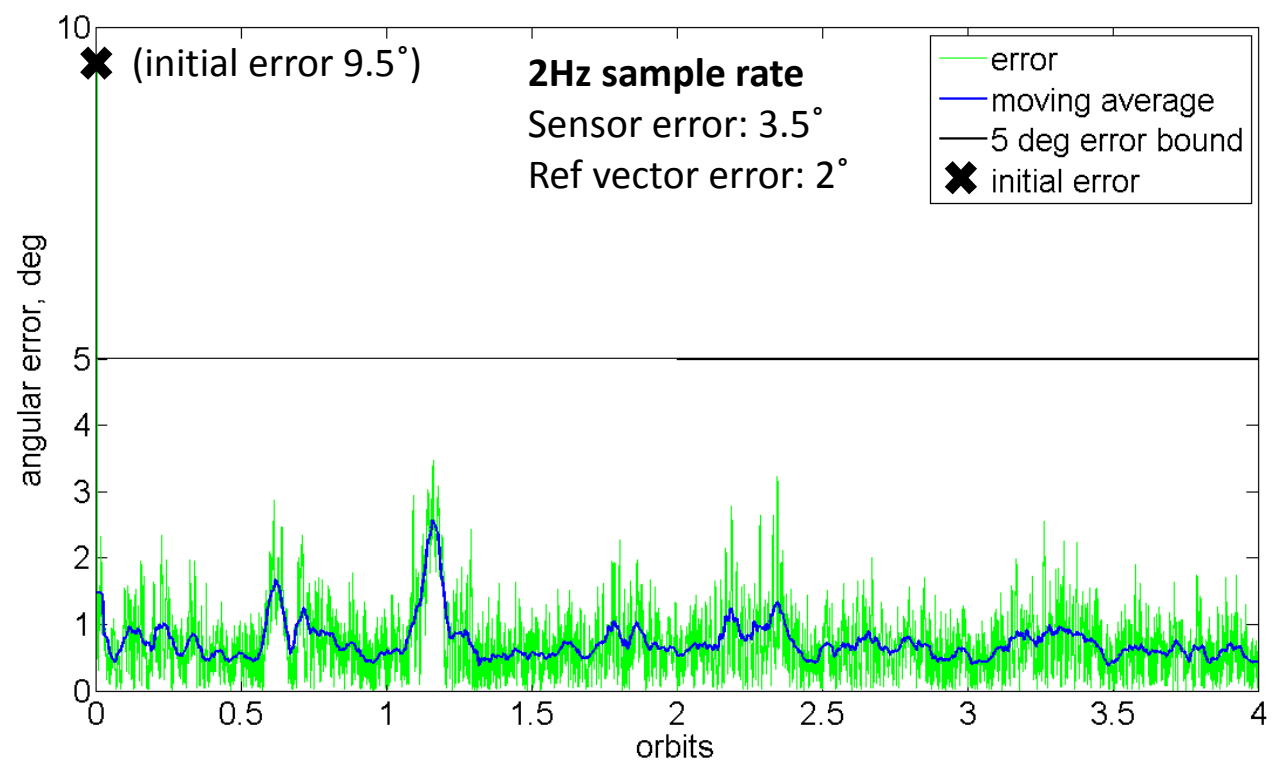

Figure 22. Science mode, $500 \mathrm{rpm}$ wheel rate, magnetometer only at $2 \mathrm{~Hz}$.

In this scenario type the EKF converges very rapidly and maintains steady state estimation well within the mission requirement. This is due mostly to the fact that the nominal orientation assumption made by the initial state $x_{0}$ is quite valid, considering the ode 45 initial state had only $5^{\circ}$ axis offsets. Initial error is just under $9.5^{\circ}$, which is also relatively low for the same reason. The periodic rises in steady state error correspond to portions of orbit in which the satellite is passing over the Earth's magnetic poles. During those portions of orbit the magnetic field is more rapidly changing, causing a rise in error as the current sample rate is evidently not fast enough to capture the rate of change of the magnetic field adequately (a rough example of aliasing). This rise is of course much more pronounced in higher inclination orbits (keeping in mind a dipole model of magnetic field), and can be lessened by increasing the algorithm sample rate, which will be explored later. 
Case 7.1.1.2: Science Mode, momentum wheel at $1500 \mathrm{rpm}$, magnetometer only, $2 \mathrm{~Hz}$ sample rate

EKF initial state: $x_{0}=[0.002,0.002,0.002,0,0,0,1]^{T}$ (Same as previous)

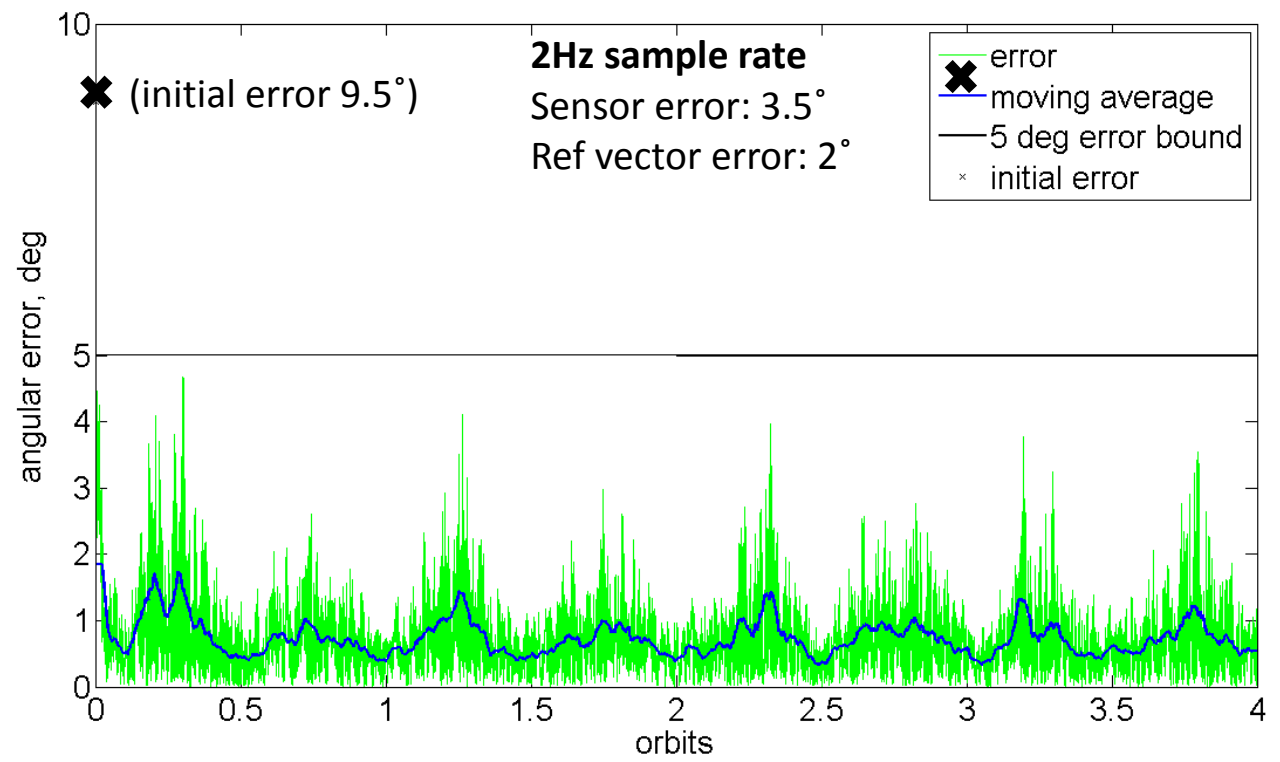

Figure 23. Science mode, $1500 \mathrm{rpm}$ wheel rate, magnetometer only at $2 \mathrm{~Hz}$.

The only simulation parameter changed with respect to the previous case is momentum wheel rate, but its effects are clear. The significantly faster rotation of the wheel causes a higher frequency spacecraft jitter, resulting in slightly coarser attitude estimation using the same sample rate - this in turn results in much more pronounced rises in error when passing over the Earth's magnetic poles when compared to the previous simulation case. Thus far the momentum wheel is not anticipated to be spun faster than this for maintained operation (for the sake of reliability) unless it is used for torqueing about the pitch axis. Therefore the rate used here presents a "worst case" with regards to its effects on steady state attitude estimation. 
Case 7.1.1.3: Science Mode, momentum wheel at $1500 \mathrm{rpm}$, magnetometer and sun sensor, TRIAD to initialize, $2 \mathrm{~Hz}$ sample rate

EKF initial state: $x_{0}=\left[0.002,0.002,0.002, q_{1 T}, q_{2 T}, q_{3 T}, q_{4 T}\right]^{T}$. Same body rates as previous case, TRIAD used to calculate initial quaternion set.

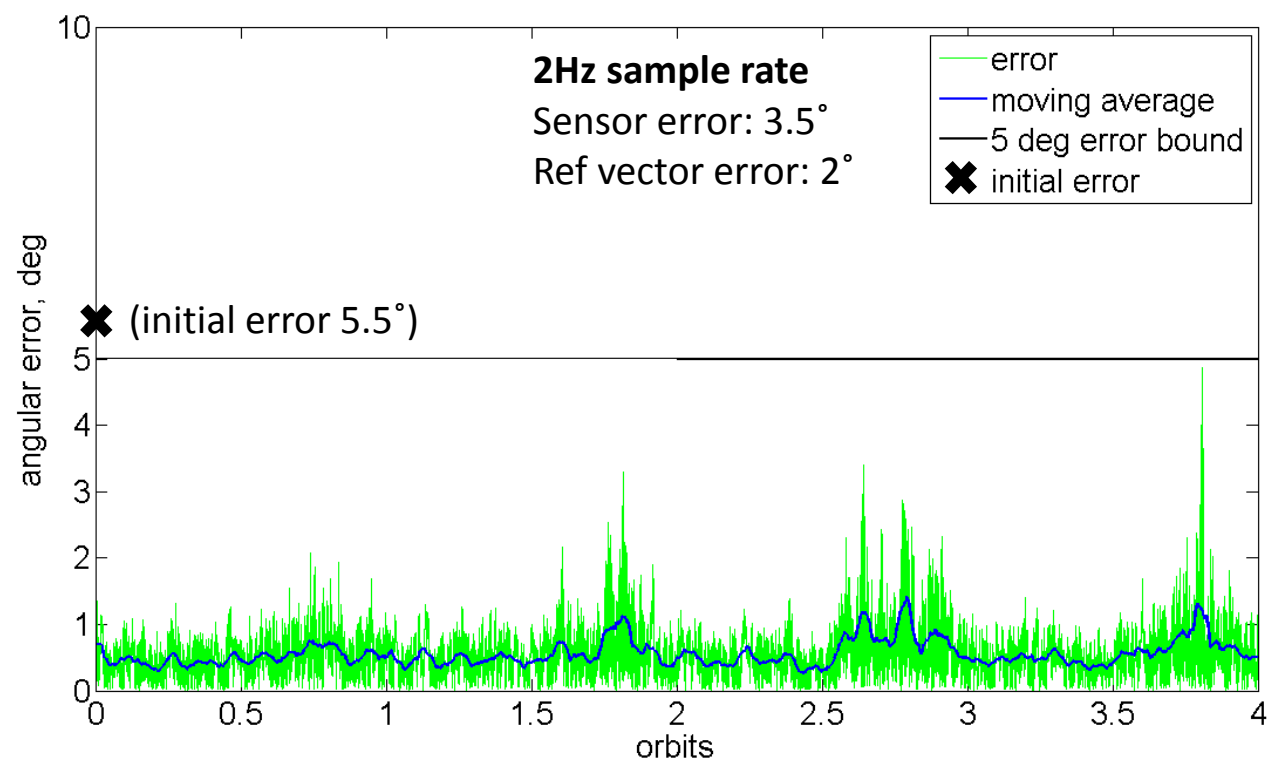

Figure 24. Science mode, $1500 \mathrm{rpm}$ wheel rate, magnetometer and sun sensor at $2 \mathrm{~Hz}$, TRIAD.

The use of TRIAD and addition of the sun sensor of course result in more rapid convergence with a lower initial error, and lower overall steady state attitude estimation. However, the error rises due to passes over the Earth's poles (when only the magnetometer is reading) is increasing in magnitude periodically in this case. This presents the need to keep the EKF in check by some means of detecting these rises and resetting when a desired level of error is exceeded. This is also a good example of why any implemented EKF should be tuned well not only to its specific application but also 
mode of operation. One such "tuning" solution explored in this case can be an increase in sample rate, presented next.

Case 7.1.1.4: Science Mode, momentum wheel at $1500 \mathrm{rpm}$, magnetometer and sun sensor, TRIAD to initialize, $4 \mathrm{~Hz}$ sample rate

EKF initial state: $x_{0}=\left[0.002,0.002,0.002, q_{1 T}, q_{2 T}, q_{3 T}, q_{4 T}\right]^{T}$ Same body rates as previous case, TRIAD used to calculate initial quaternion set.

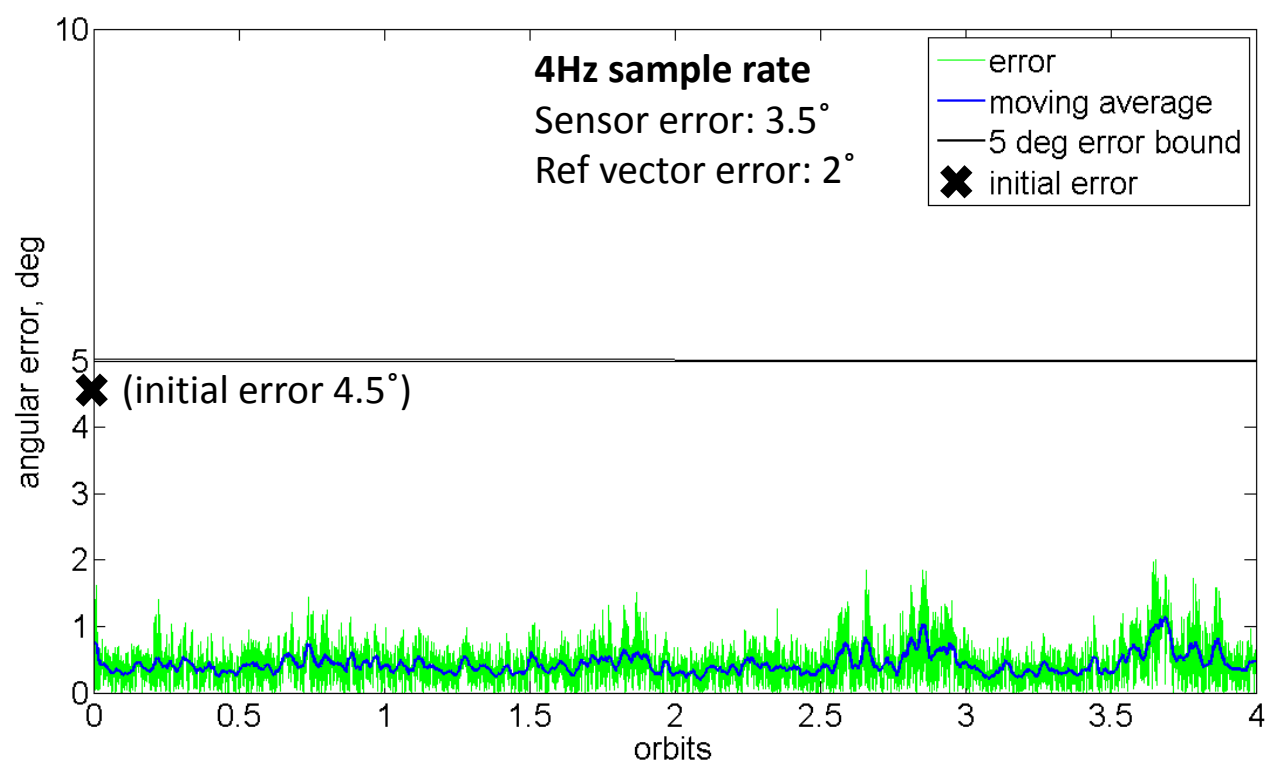

Figure 25. Science mode, $1500 \mathrm{rpm}$ wheel rate, magnetometer and sun sensor at 4Hz, TRIAD.

The increase in EKF sample rate from $2 \mathrm{~Hz}$ to $4 \mathrm{~Hz}$ has a significantly positive impact on estimation results. Figure 25 shows an average steady state error lower than $1^{\circ}$ and much lower rises when passing near magnetic poles. $4 \mathrm{~Hz}$ has thusly been chosen tentatively as the recommended EKF sample rate, as it provides attitude estimation to well within the mission required magnitude of $5^{\circ}$ knowledge, with margin for error accounted for in the 
modeling of sensors and their corresponding reference vectors. Electrical and software engineers currently working with attitude hardware and software for Exocube have stated that a $4 \mathrm{~Hz}$ read rate is within the capabilities of the sensors and flight board chosen without a loss in performance. The sample rate was not driven any higher than this in simulation due to the computational burden imposed. To explore the reliability of this simulation case, the same parameters were input to a case spanning 20 orbits. Results are shown below in Figure 26.

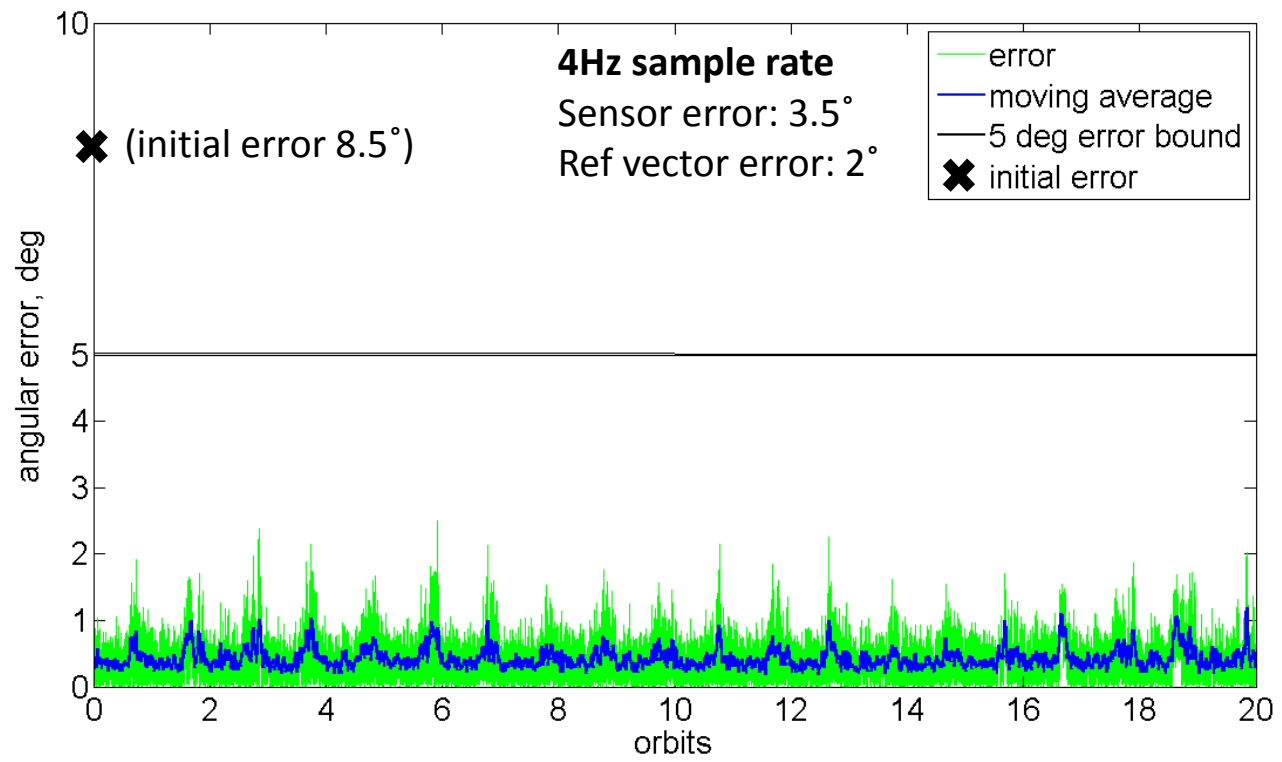

Figure 26. Science mode, $1500 \mathrm{rpm}$ wheel rate, mag/sun sensors at 4Hz, TRIAD, long duration.

Steady state estimation for the "long duration" version of this case has the same rapid convergence and average error under $1^{\circ}$. The higher initial error and higher error rises when passing over magnetic poles can be attributed to the random nature of noise incorporated in the simulation model, as their magnitudes vary slightly with each run. Nonetheless, successful operation of the EKF in the dynamic state most crucial to the 
mission requirement for attitude knowledge has been proven feasible with respect to providing the required knowledge reliably. This has been done with a relatively comfortable amount of margin, considering the error inputs to the model are higher than anticipated thus far as well as the fact that the filter has not yet been fine tuned for this specific mode of operation.

\subsubsection{Further EKF Analysis for Science Mode}

In the interest of pursuing further development of the EKF formulated in this work for the Exocube mission (as well as any future PolySat missions requesting its use), some sensitivity analysis was conducted specifically for the nominal science mode case presented previously (4 Hz sample rate). Average and maximum steady state estimation error outputs were recorded for varying input errors and for ranges of filter $P / Q$ ratios $(P$ being the diagonal elements set for $P_{0}$ and $Q$ denoting the value of its own diagonal elements when initializing the filter). "Steady state" in this section refers to data generated after three orbits; this was done so it could be confidently assumed that the EKF had settled, based on the results presented in Figure 25 and Figure 26.

The plot below characterizes the relationship between sensor and reference vector error inputs and EKF steady state estimation error. 


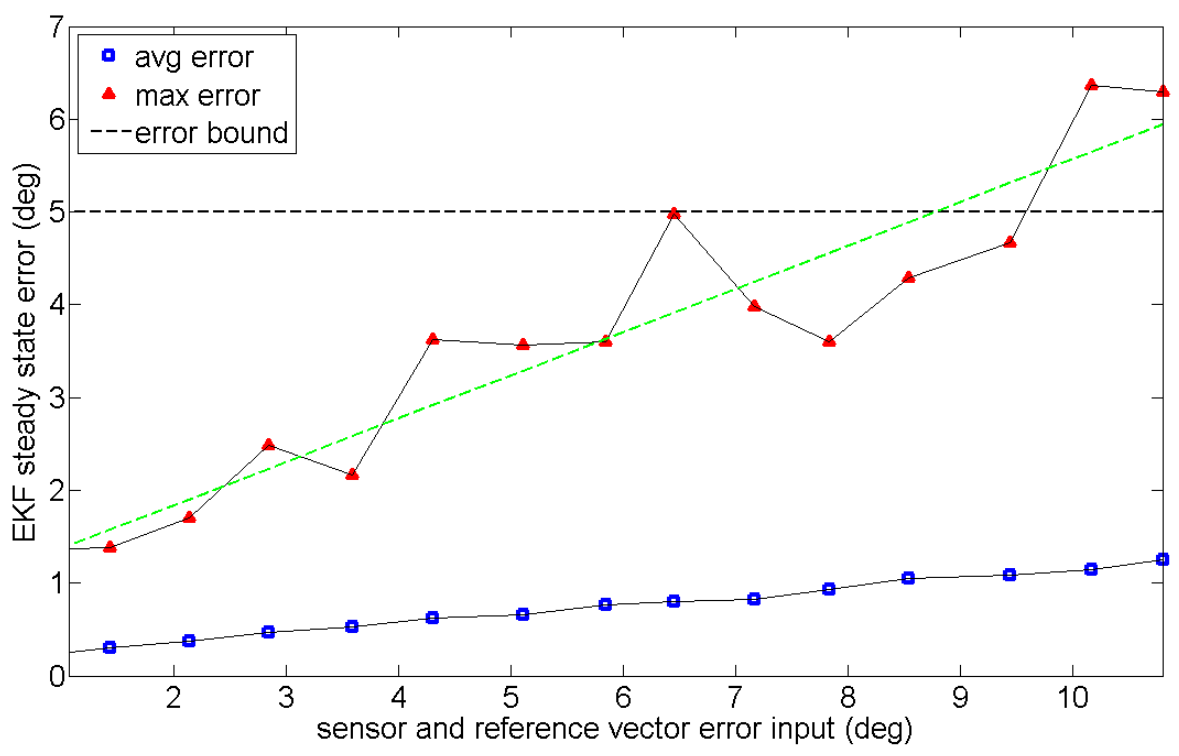

Figure 27. EKF steady state error resulting from varying input errors.

As mentioned earlier, maximum error is a product of magnetometer-only sensor readings while passing over magnetic poles, and is somewhat random in magnitude with every simulation run due to the random nature of the error inputs. Nonetheless, it still shows an increasing trend with increasing input error, as does average estimation error. Because the average estimation error remains rather low and grows and at much slower rate than maximum error, it is clear that the amount of input error the EKF in this work can manage while providing attitude knowledge to within the mission requirement will be driven more so by maximum output error. For the case of Exocube, in the event that the error bound margin is narrowed greatly - whether it be due to a lower sample rate or increased vector input errors - it will be up to the ADCS team to coordinate with the Exos payload developers to negotiate which portions of orbit accurate science data is most desired. If in the dark, which corresponds to magnetometer-only readings, then 
additional work would have to be done to ensure a safe margin between the maximum resultant errors and knowledge requirement error bound.

Moving on, maximum and average steady state estimation errors were explored for the aforementioned varying $P / Q$ ratio. In Figure 28, this ratio was ranged while holding $Q$ constant.

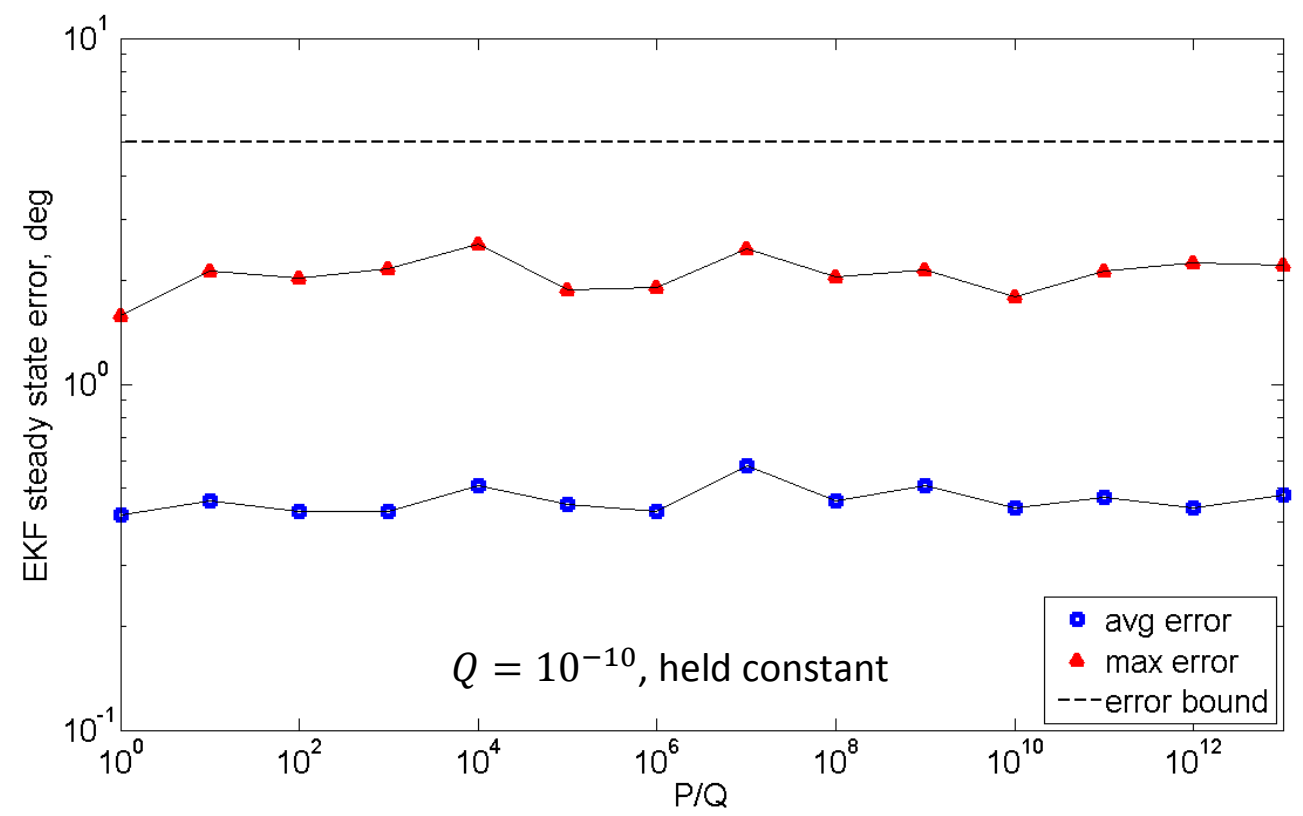

Figure 28. EKF steady state error with varying P/Q ratio, $Q$ held constant (1e-10).

Varying the initial diagonal elements of the error covariance matrix $P$ does not appear to have much effect on the EKF steady state estimation error, as shown by the flat nature of the plots. It can therefore be stated that at least for the nominal science mode dynamic state, the EKF is rather robust with respect to the initialization of the $P$ matrix. Ranges of $P / Q$ ratios, this time with a constant $P$ and varying process noise matrix value $Q$ were run next. Figure 29 plots results for varying $Q$ while holding $P$ constant at $10^{-10}$. 


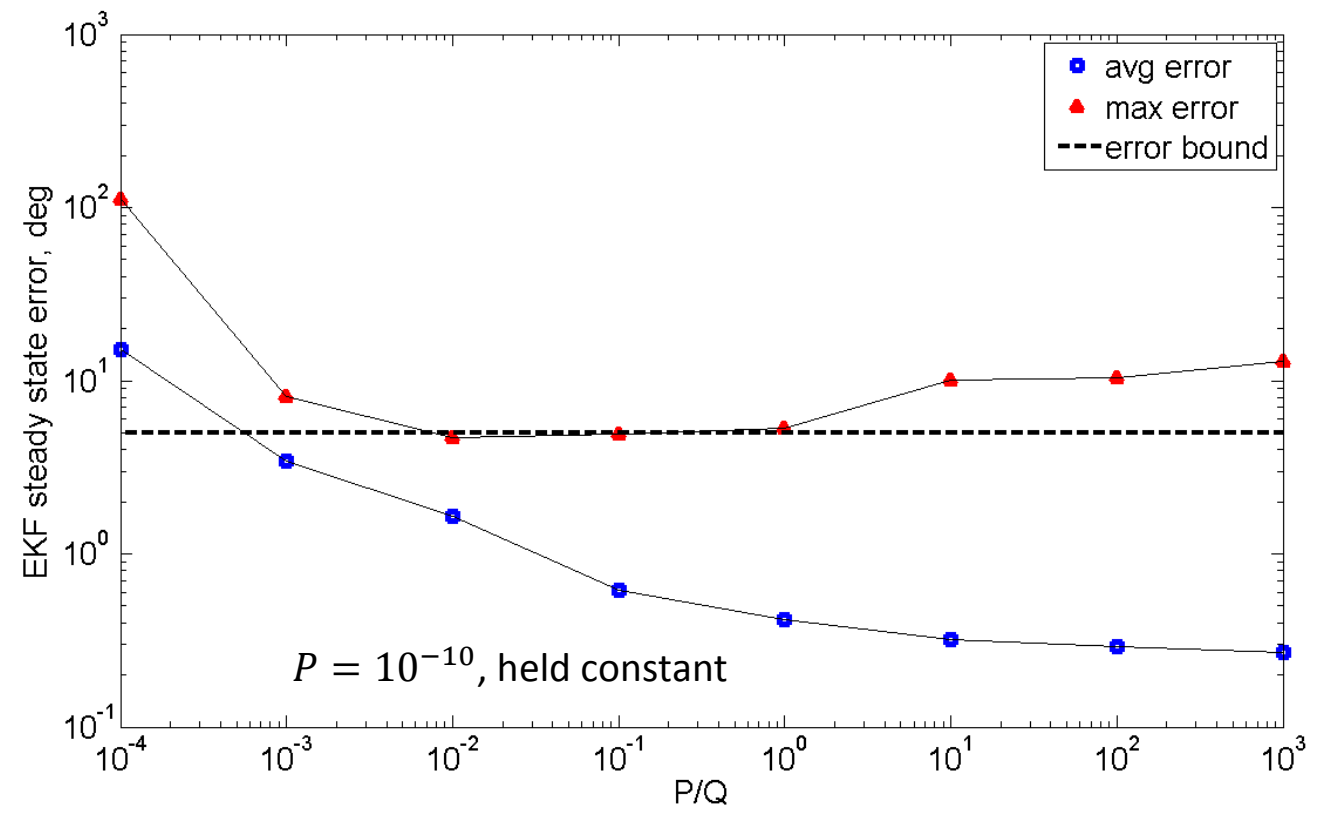

Figure 29. EKF steady state error with varying $P / Q$ ratio, $P$ held constant (1e-10).

Varying $Q$ clearly has more of an effect on the performance of the EKF. It can be seen here that given points within a set range of ratios involve a compromise between levels of average and maximum errors, thus validating the need for tuning of the filter for optimum performance. One more such case was generated, this time varying $P / Q$ ratio with $P$ held constant at a value of one. Results are shown below. 


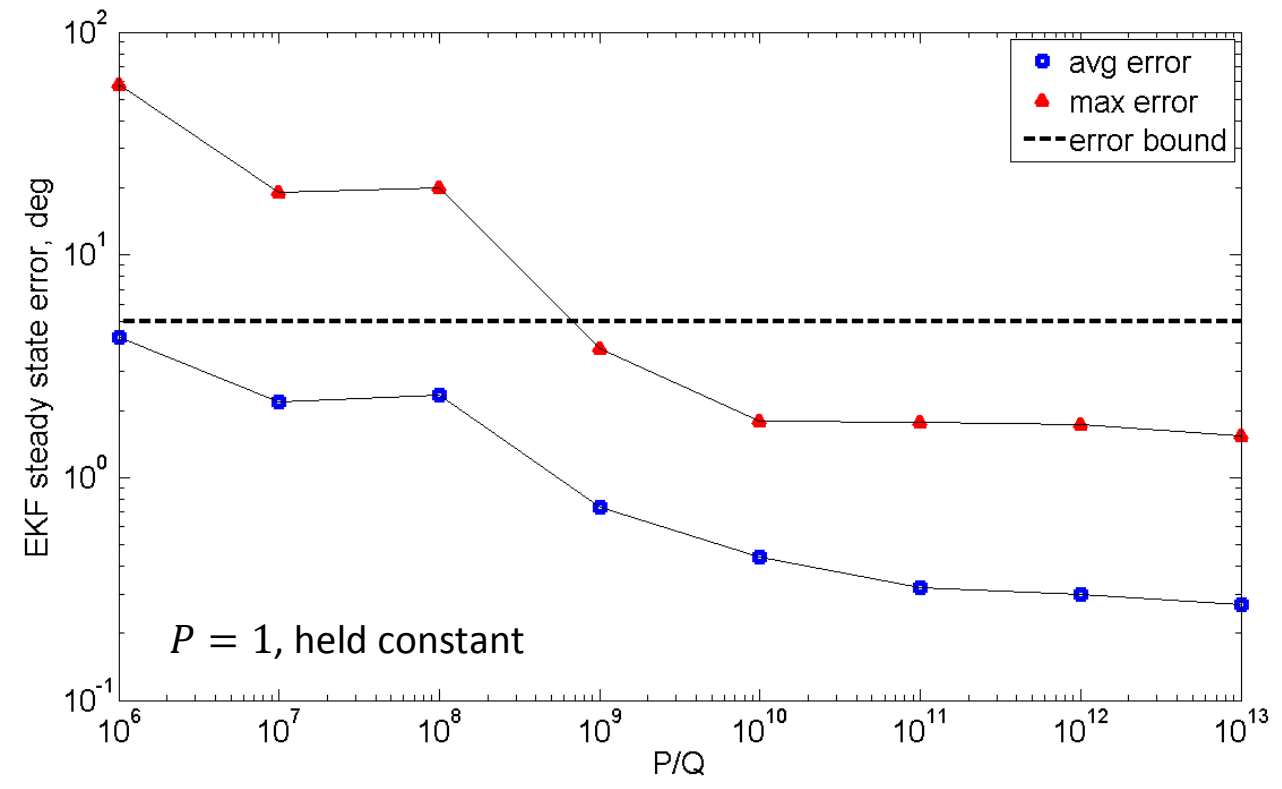

Figure 30. EKF steady state error with varying $P / Q$ ratio, $P$ held constant (1e0).

It is apparent from Figure 30 that ranging of $Q$ has a much different consequence with a different value set for $P$. It is very interesting to note here that when compared to the previous plot, for the range $10^{-13} \leq Q \leq 10^{-9}$ the EKF is essentially performing better with a higher error covariance initialization. Therefore although varying the initialization parameter $P$ at first appeared inconsequential in Figure 30, it is now clear that its value coupled with that set for $Q$ can greatly affect EKF performance (keep in mind that this is all assuming uniform diagonal elements for initialization, which isn't necessarily optimal). It can be concluded here that the three cases presented here for $P / Q$ ratio have barely scratched the surface of the potential for analysis and tuning of the filter itself. It would be wise for future work to at the very least characterize EKF steady state performance with the varying of both $P$ and $Q$ values simultaneously, obtaining results 
similar to those seen in this section but in a more all-encompassing format - perhaps in the form of a contour/surface plot.

\subsubsection{EKF Science Mode Conclusions}

It has been demonstrated in Section 7.1.1 Science Mode through simulation and analysis that the selection and specific formulation of attitude algorithms in this work do in fact have the potential to provide Exocube the mission required attitude knowledge of $\pm 5^{\circ}$ in an operational mode governed by the mission requirements for attitude control. The algorithms can in turn be implemented on board Exocube to give real time attitude knowledge corresponding to times at which the instrument payload is taking data, thereby validating its measurements by ensuring a known bound of attitude knowledge. Having performed well with regards to this crucial mission phase, it was then desired to apply these simulations and algorithms to other anticipated dynamic states of the Exocube mission. The following sections provide some insight as to how the EKF performed when applied to these differing states.

\subsubsection{Post Deployment, Pre Gravity Gradient Capture}

This specific scenario describes the motion of Exocube after initial deployment from its launch vehicle interface (the Cal Poly P-POD). The momentum wheel is off and the satellite is assumed to be tumbling at a rate slightly higher than $1 \%$ about all axes. The satellite is also off of nominal orientation in every axis by $60^{\circ}$, setting the ode 45 initial state vector to $x=[0.02,0.02,0.02,0.156,0.592,0.159,0.775,0,0,0,0]^{T}$ (body rates are expressed in $\mathrm{rad} / \mathrm{s}$ ). The resulting spacecraft dynamics are shown in the series of figures below. 


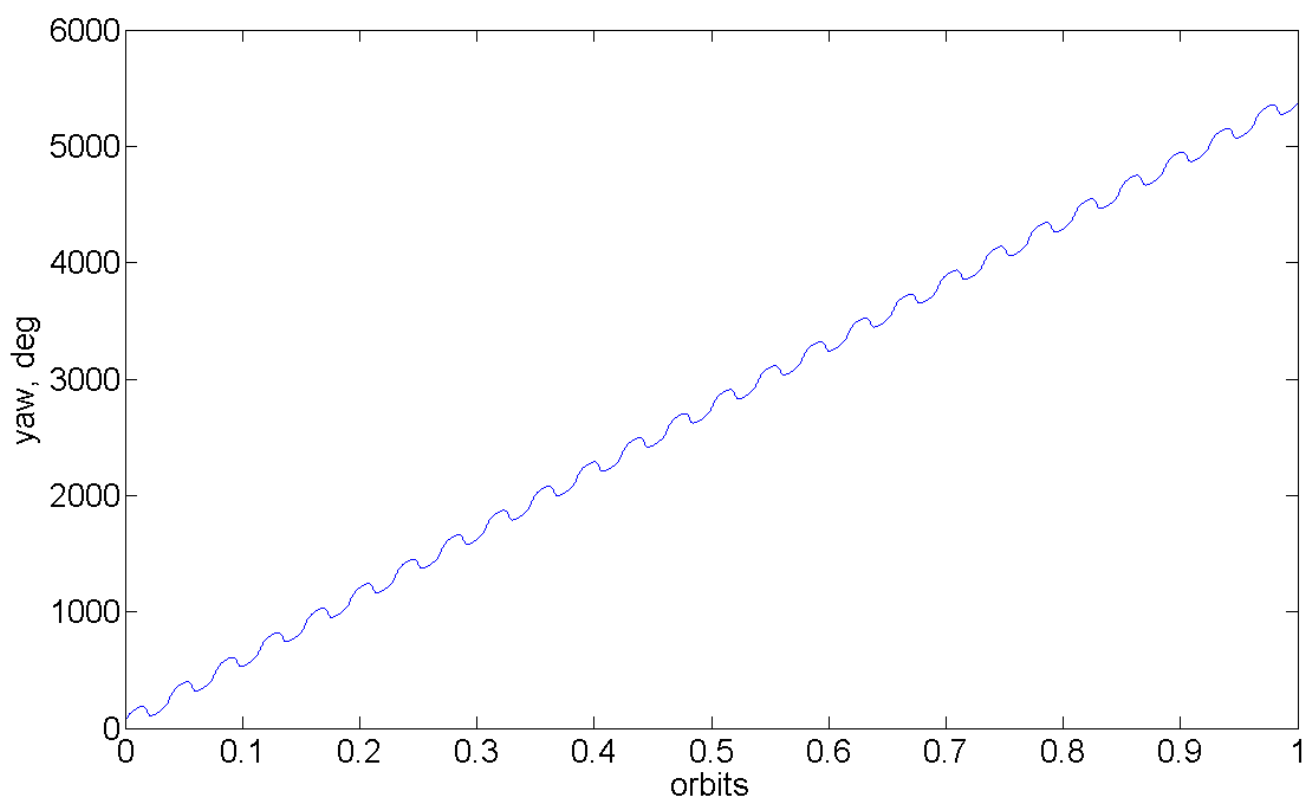

Figure 31. Tumble scenario dynamics, yaw axis (b1).

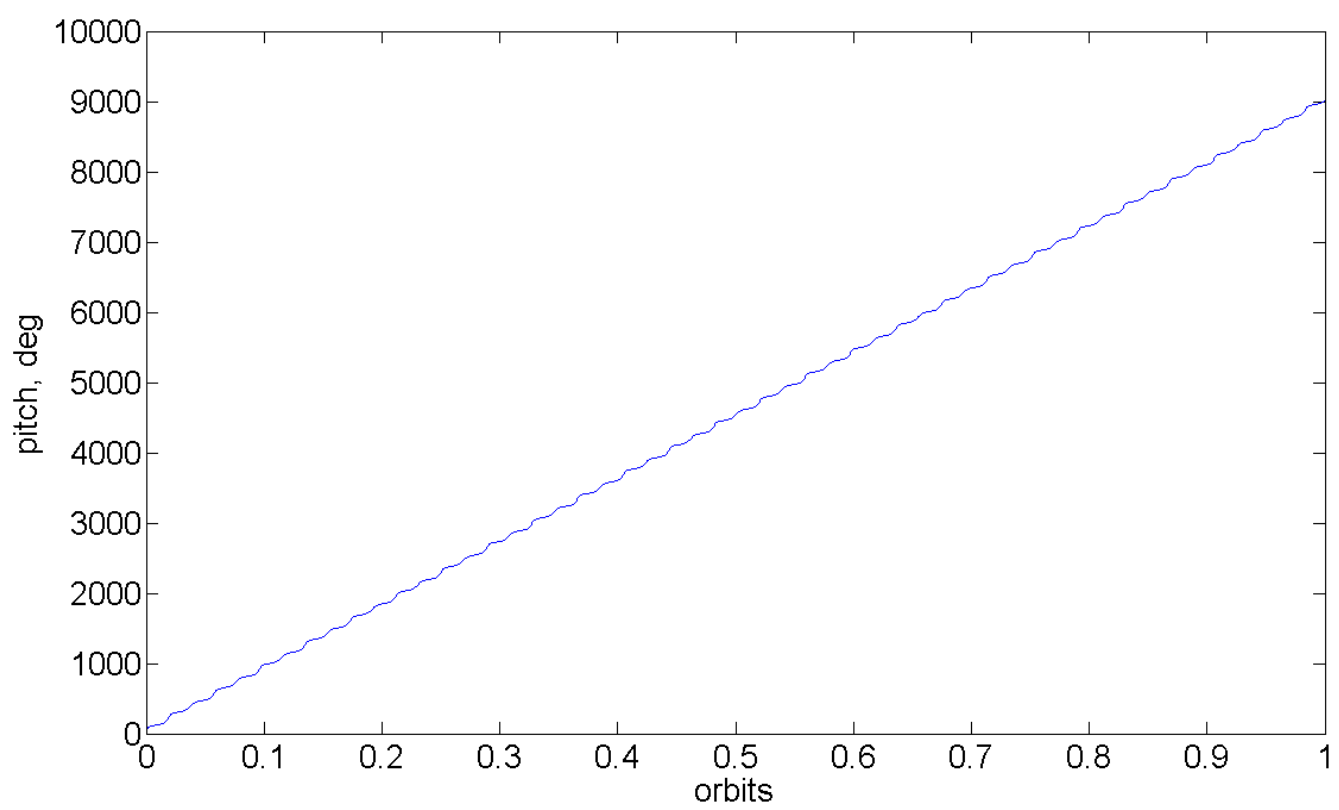

Figure 32. Tumble scenario dynamics, pitch axis (b3). 


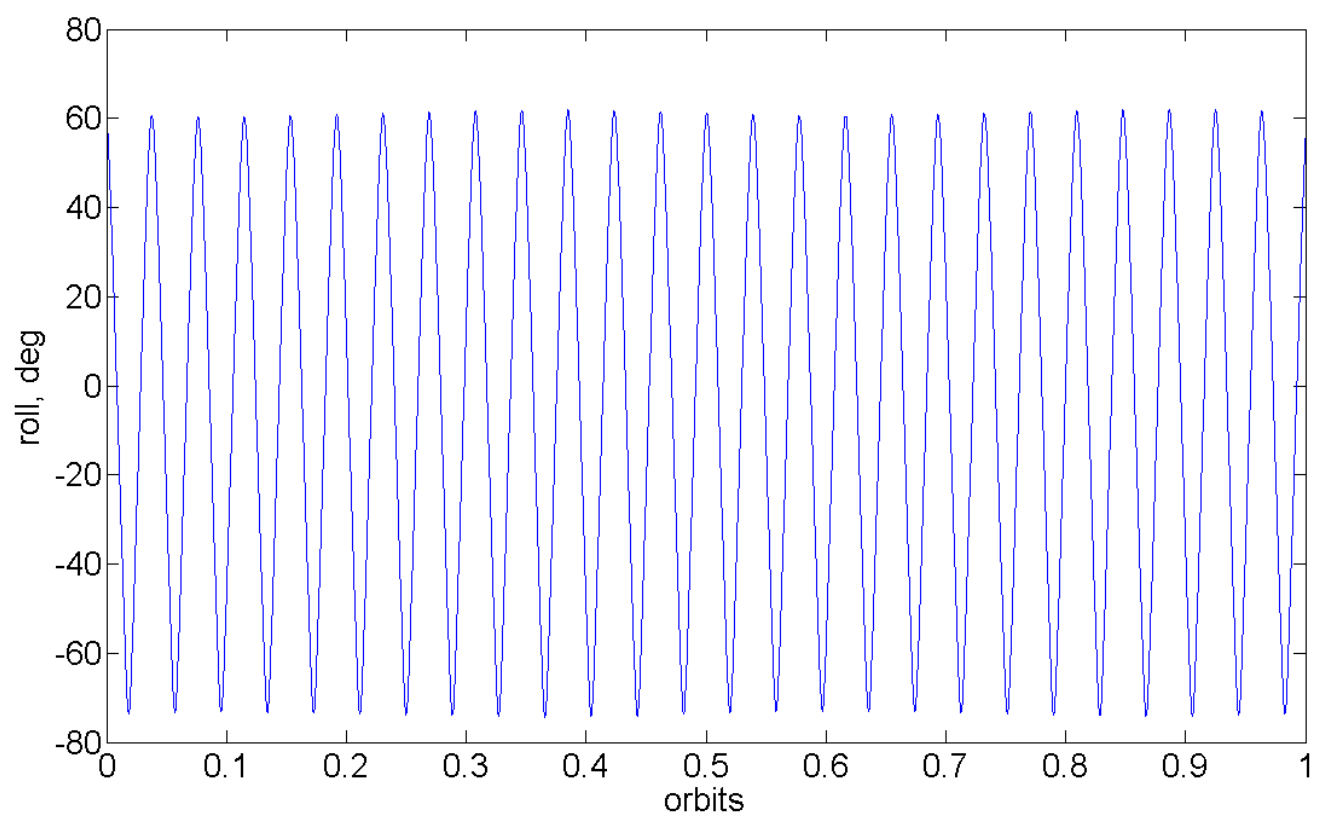

Figure 33. Tumble scenario dynamics, roll axis (b2).

Figure 31 and Figure 32 clearly demonstrate the instability of the system in this dynamic state, as the yaw and pitch axes spin freely over time. The roll axis oscillates about zero (albeit with a high amplitude) due to the fact that it is being acted on by a gravity gradient torque but without the direct effect of inherent orbital motion (see Equations (14), (26)). Initial state vectors given to the EKF will be explained briefly with each case run.

Case 7.1.2.1: Post-Deploy Tumble, magnetometer only, $2 \mathrm{~Hz}$ sample rate.

EKF initial state: $x_{0}=[0.04,0.04,0.04,0,0,0,1]^{T}$. Body rates not matching those in ode 45 but same order of magnitude, quaternions assume body begins in nominal orientation. 


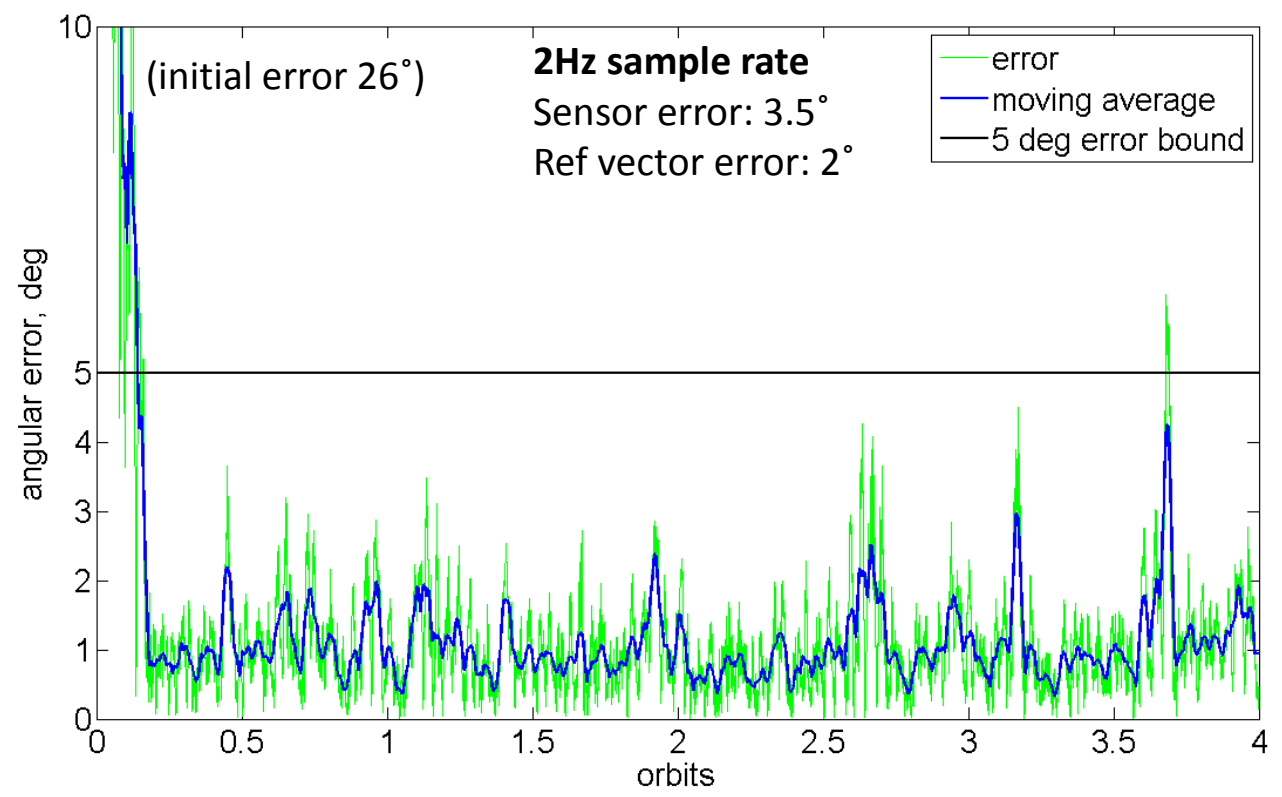

Figure 34. Post deployment, magnetometer only at $2 \mathrm{~Hz}$.

The magnetometer only case converges before a quarter of its first orbit, maintaining steady state estimation afterwards to within $5^{\circ}$ until about 3.75 orbits. This spike in error along with the other more pronounced ones seen around 2.75 and 3.25 orbits are due to the satellite passing close to the Earth's poles, as described in the previous results section. Error prior to convergence is in fact $26^{\circ}$, but the y-axis limit was forced on the plot to give a more detailed view of steady state error (this also applies to most of the cases to come). Robustness of the filtering algorithm is made apparent here by convergence despite an initial quaternion set that is $60^{\circ}$ off of every axis compared to the actual initial state input to ode45. 
Case 7.1.2.2: Post-Deploy Tumble, magnetometer and sun sensor, $2 \mathrm{~Hz}$ sample rate

EKF initial state: $x_{0}=[0.04,0.04,0.04,0,0,0,1]^{T}$. (Same as previous)

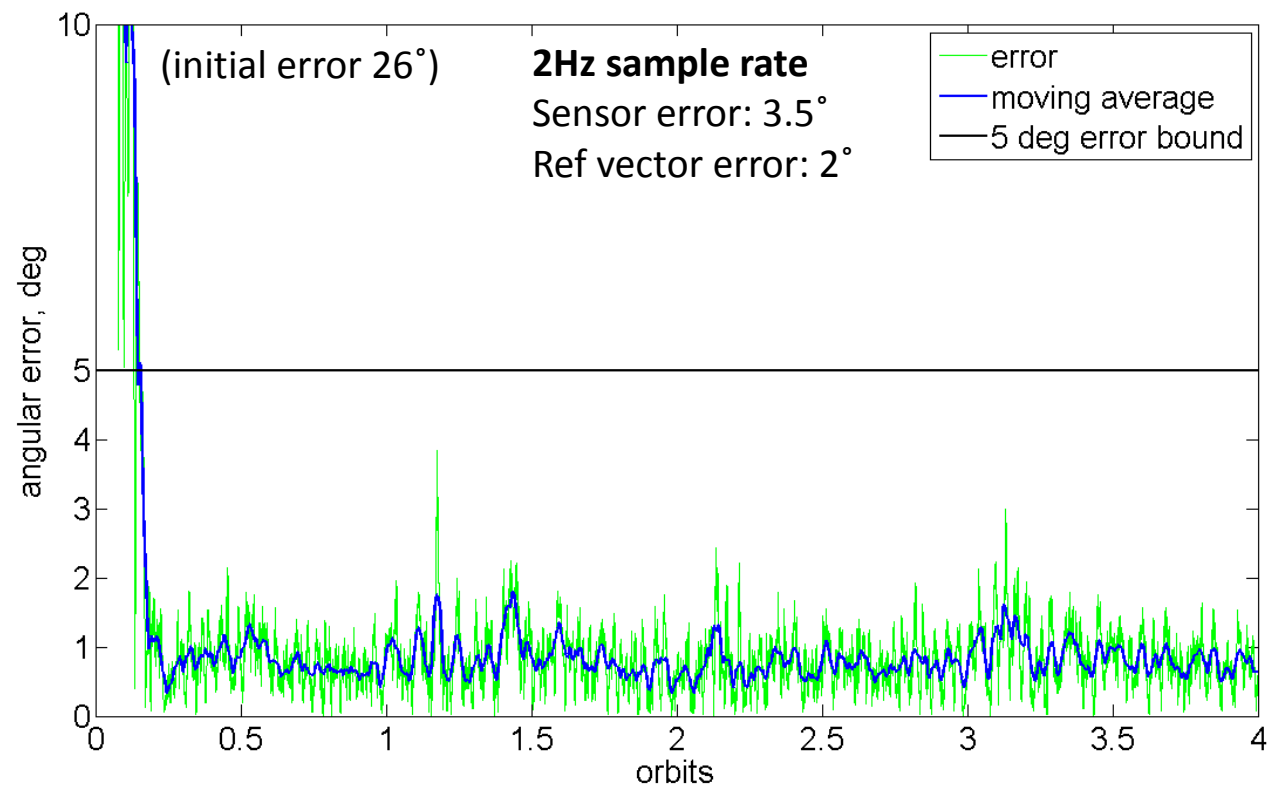

Figure 35. Post deployment, magnetometer and sun sensor at $2 \mathrm{~Hz}$.

The additional use of a sun sensor every half orbit reduces average steady state estimation error, and will also reduce convergence time if the algorithm is started in sunlit conditions. In this case the algorithm was started in eclipse conditions however, and as such performs similarly to the previous case with regards to initial error and convergence time (since no parameters were altered). The sharp error peaks seen in the magnetometeronly case are lessened, but periodic rises in error are still seen due to sensor switching in sunlit to eclipse transitions as well as magnetometer-only readings when passing over Earth's magnetic poles. 
Case 7.1.2.3: Post-Deploy Tumble, magnetometer and sun sensor, TRIAD to initialize, $2 \mathrm{~Hz}$ sample rate

EKF initial state: $x_{0}=\left[0.04,0.04,0.04, q_{1 T}, q_{2 T}, q_{3 T}, q_{4 T}\right]^{T}$. Same body rates as previous case, TRIAD used to calculate initial quaternion set.

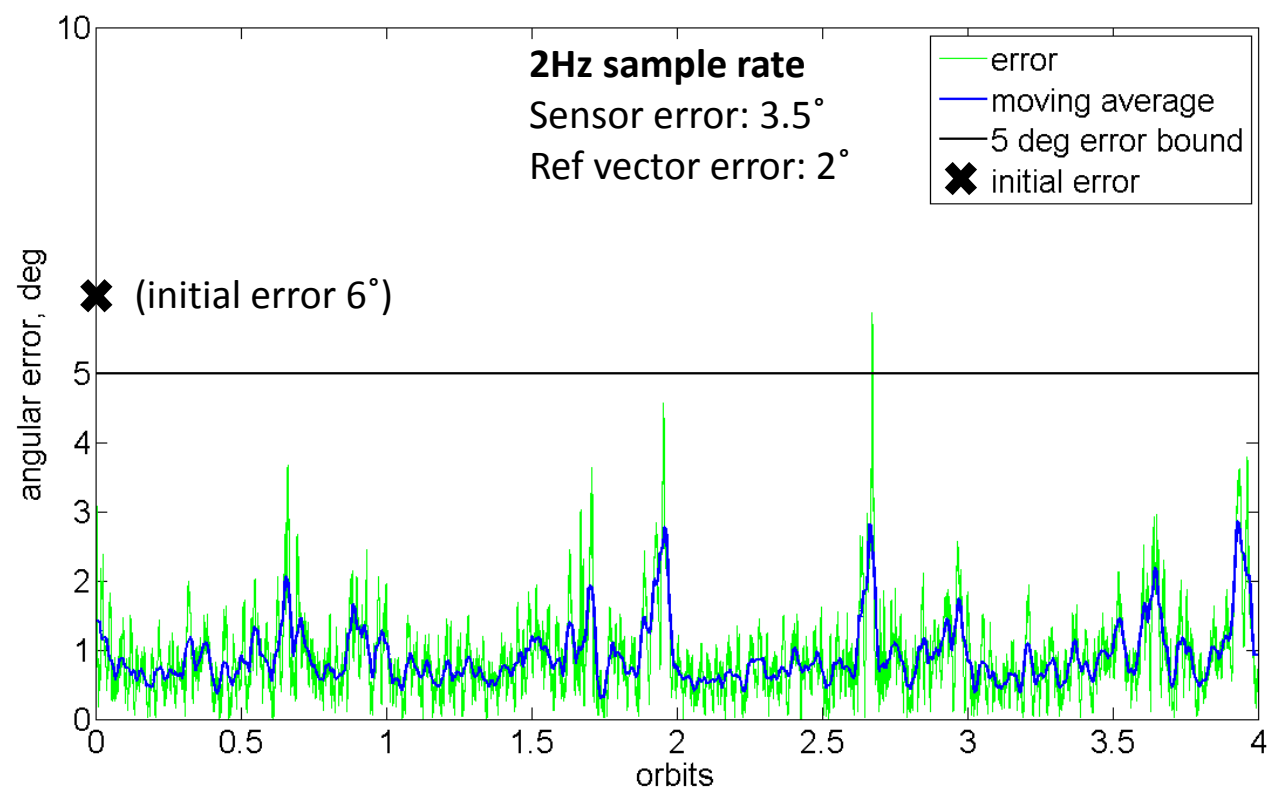

Figure 36. Post deployment, magnetometer and sun sensor at 2Hz, TRIAD.

The use of TRIAD to aid in initialization of the EKF results in almost immediate convergence. Steady state error in this case is not nearly as smooth as it was without the use of TRIAD, however. This may be due to the lower error initial state causing the EKF to rely more heavily on its propagation phase throughout its use (Vallado calls this "filter smugness"). ${ }^{10}$ Obviously, the filter needs at least some of its initial parameters tuned differently for this case to aid in steady state estimation accuracy and consistency. A higher sampling rate may also alleviate the issue. As a final note, the use of TRIAD in 
this case of course means that the attitude algorithms would need to be started up in the sunlit portion of orbit.

\subsubsection{Gravity Gradient Capture / Perturbed Science Mode}

This scenario describes Exocube in two different dynamic states, depending on whether or not the momentum wheel is on. With the wheel off it can be considered in its gravity gradient captured state, in which magnetorquers have been utilized to damp body rates to a "low enough" level and the gravity gradient booms have been deployed. With the wheel on, the satellite can be modeled as perturbed slightly off of its control requirement parameters as defined in Section 1.1 Background. Both of these case types use the same dynamic simulation state vector. A rate disturbance of $0.1 \%$ is applied to all axes, and the satellite body starts at $20^{\circ}$ degrees off of nominal orientation in all axes. This sets the attitude simulation initial state vector to $x=[0.0018,0.0018,0.0018,0.139,0.198$, $0.139,0.960,0,0,0,0]^{T}$. Spacecraft body rates for the gravity gradient capture state (momentum wheel off) are shown below. 


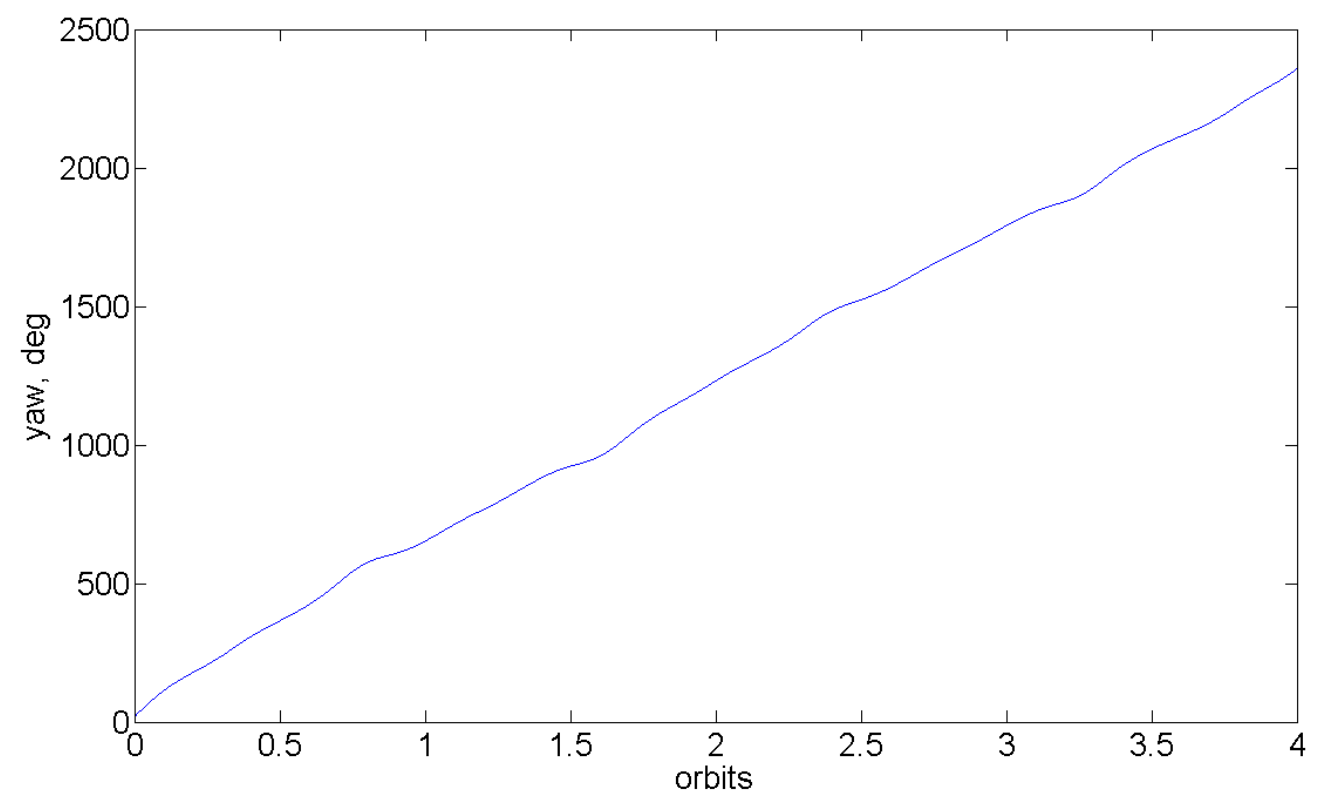

Figure 37. Gravity Gradient Capture scenario dynamics, yaw axis (b1).

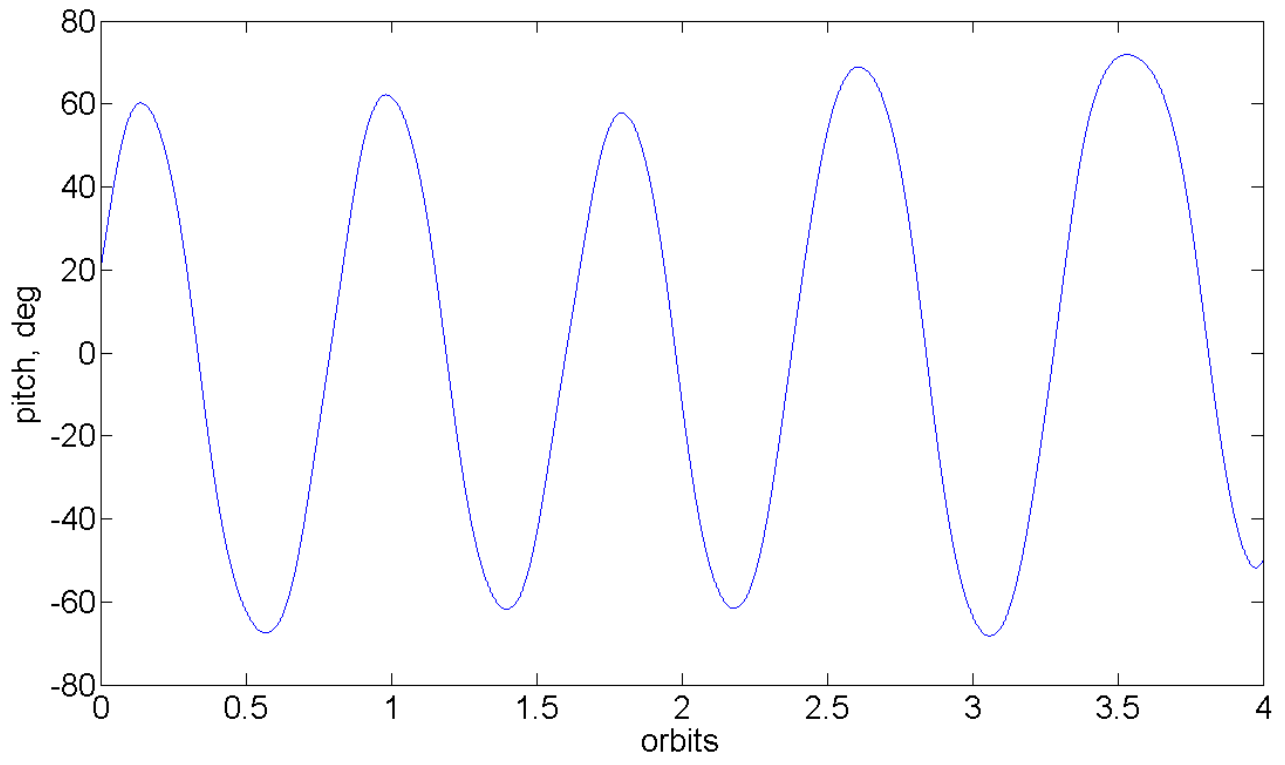

Figure 38. Gravity Gradient Capture scenario dynamics, pitch axis (b3). 


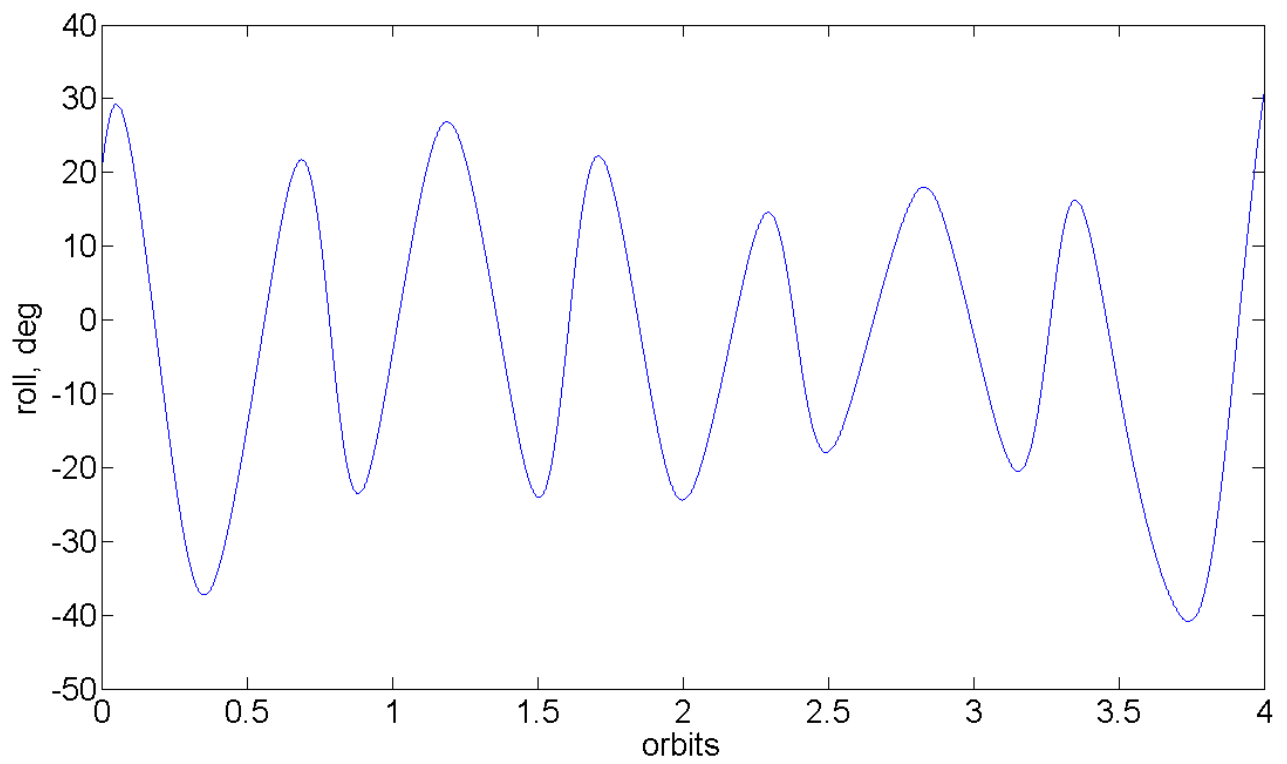

Figure 39. Gravity Gradient Capture scenario dynamics, roll axis (b2).

Figure 37 shows the yaw instability inherent in a gravity gradient only system, while the large amplitude of oscillation present in Figure 38 and Figure 39 given the relatively small disturbance inputs is due to the small inertias of the satellite. Body rates are shown below for the same initial state inputs but with the momentum wheel at $1500 \mathrm{rpm}$ (perturbed science mode). 


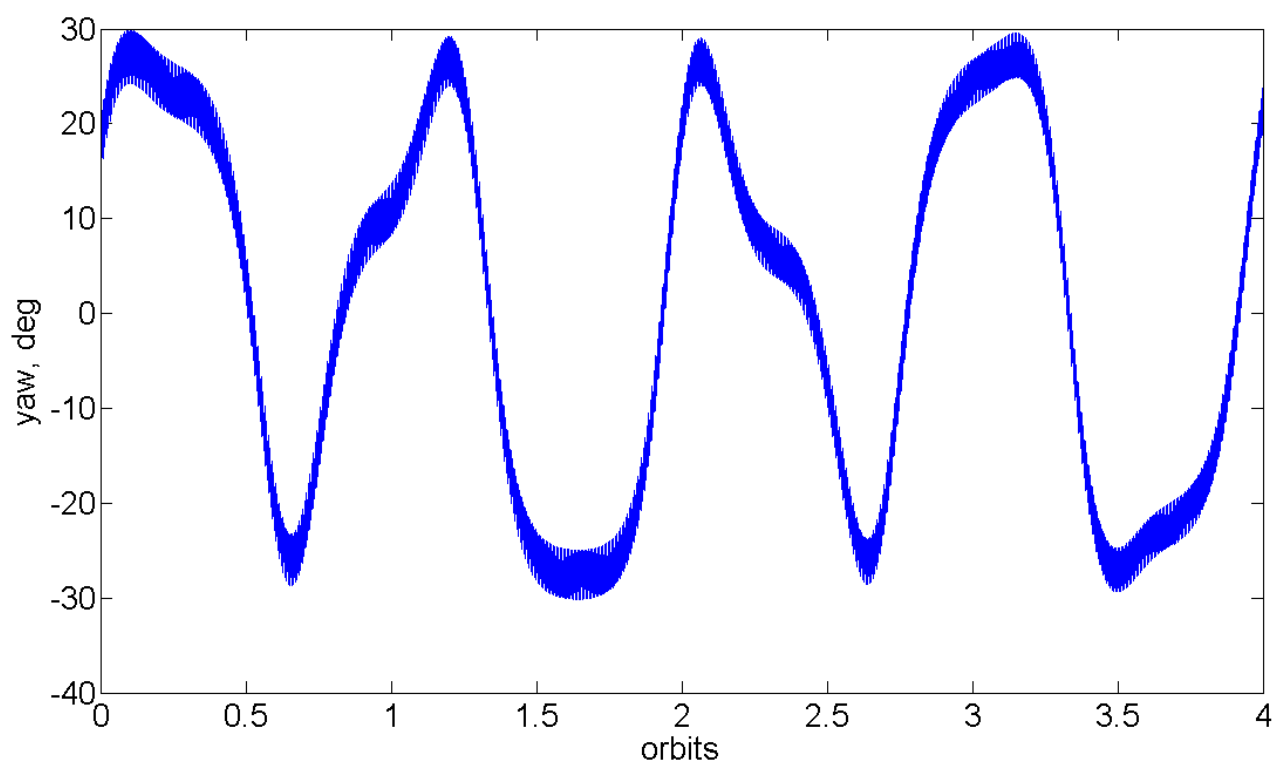

Figure 40. Perturbed Science scenario dynamics, yaw axis (b1).

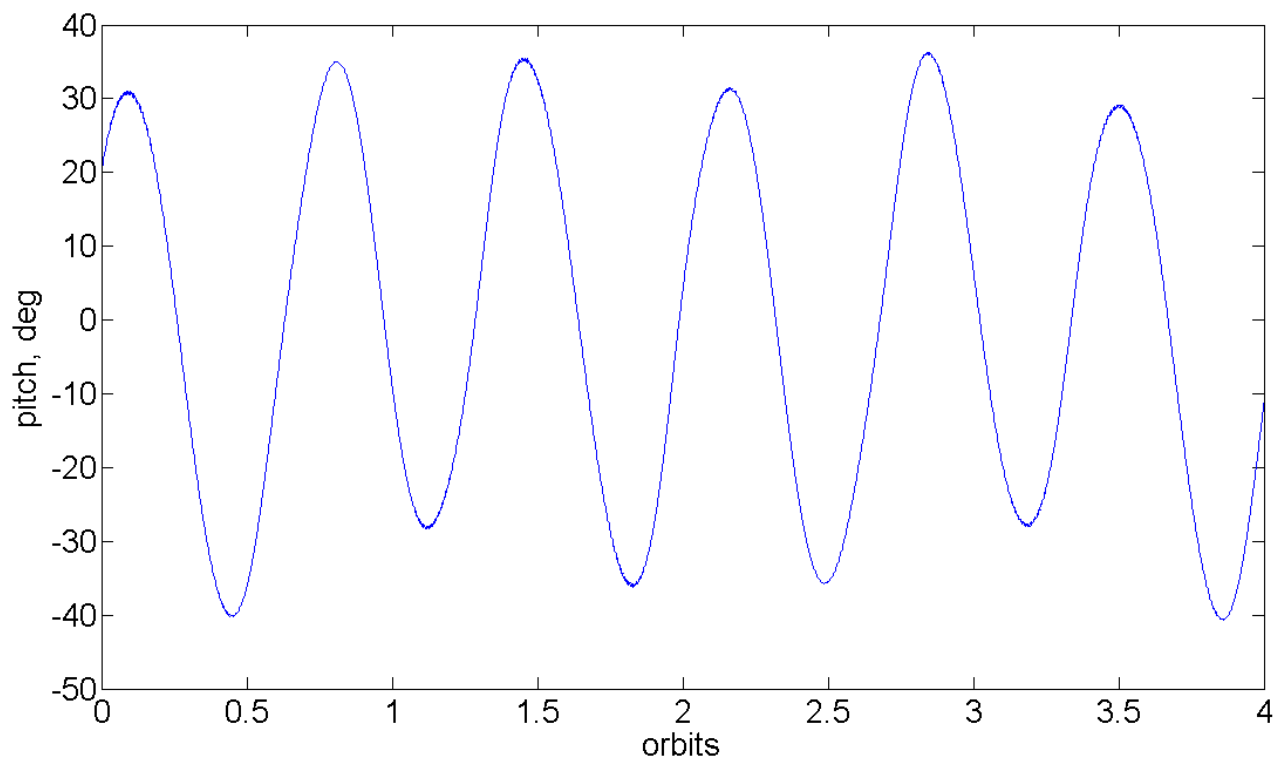

Figure 41. Perturbed Science scenario dynamics, pitch axis (b3). 


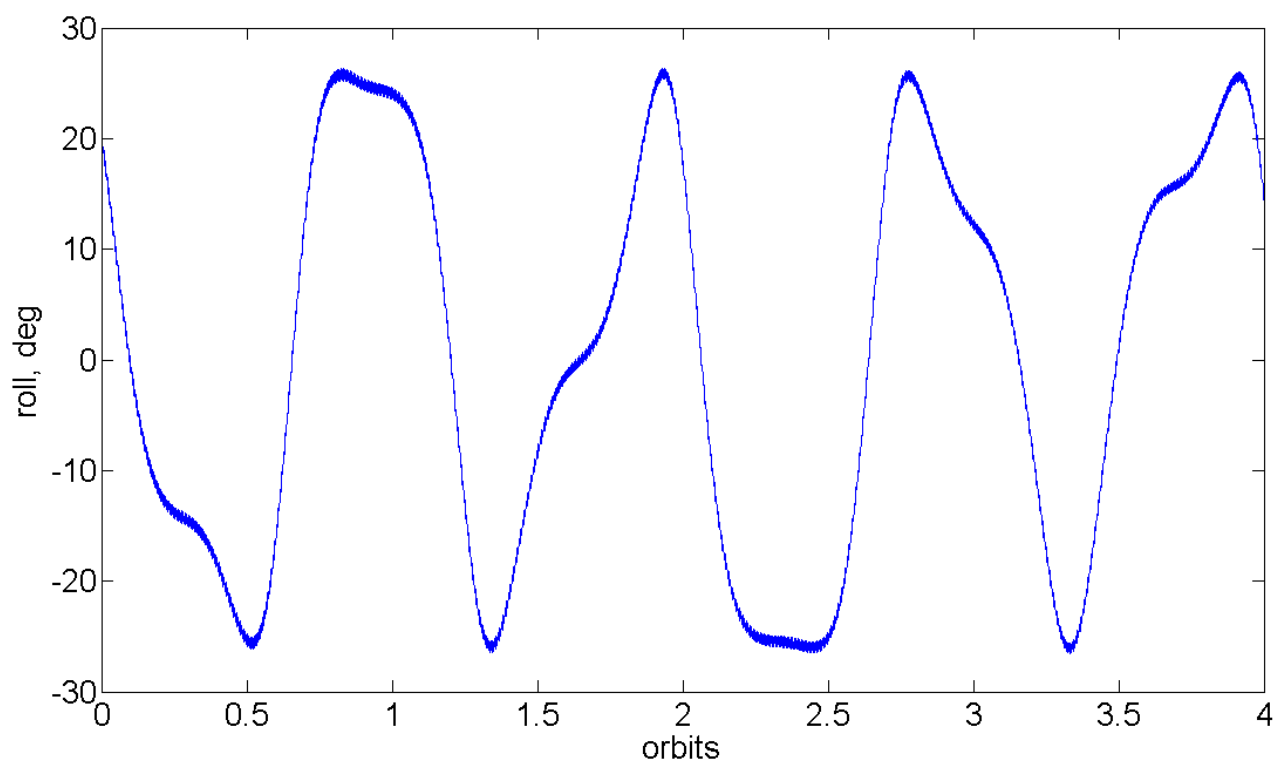

Figure 42. Perturbed Science scenario dynamics, roll axis (b2).

The use of the momentum wheel clearly adds stability to the spacecraft body yaw axis, as it is no longer rotating freely. Oscillation amplitudes have been reduced, and the jitter described in Section 3.2.2 Momentum Wheel - Gyrostat Equations is present in the coupled yaw and roll axes, as depicted by the varying thickness of plot lines. Simulations for these dynamic states were run at different step times, since performance of the EKF was driven heavily by a longer sample rate for the gravity gradient capture dynamic state.

Case 7.1.3.1: Gravity Gradient Capture, momentum wheel off, magnetometer only, $0.1 \mathrm{~Hz}$ sample rate

EKF initial state: $x_{0}=[0.005,0.005,0.005,0,0,0,1]^{T}$. Body rates set higher, nominal orientation assumed. 


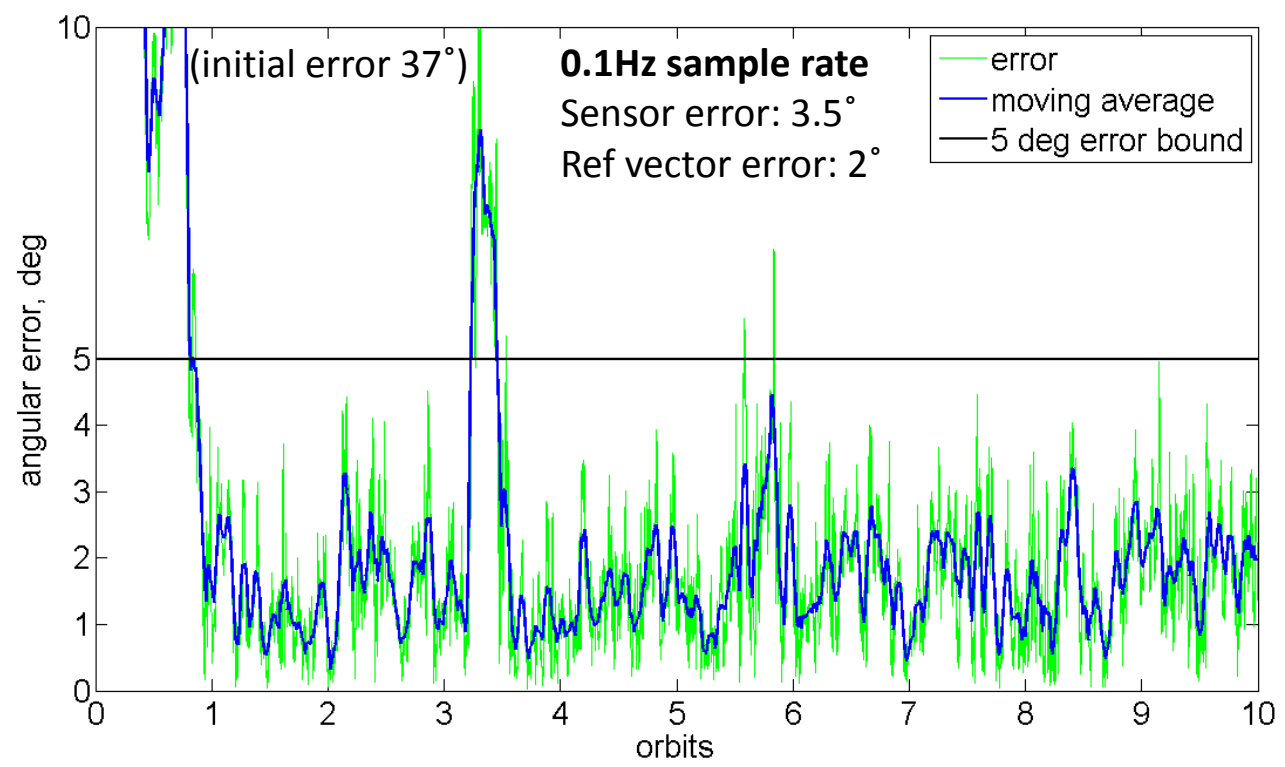

Figure 43. Gravity Gradient Capture, magnetometer only at $0.1 \mathrm{~Hz}$.

The gravity gradient capture dynamic case was somewhat peculiar with regards to EKF performance. Sample rate had to be decreased to $0.1 \mathrm{~Hz}$ to obtain convergence, which is not reached for about six orbits. The need for this lower sample rate is likely due to the calmer dynamics of the system, as presented by Figure 37, Figure 38, and Figure 39. For a faster sample rate, no tumbling or jitter causes measurements taken to be close to those estimated, quickly driving the Kalman gain too low and causing the filter to not utilize its measurement update as it should. With the lower sampling rate, there is more change in attitude dynamics in between measurements and the issue is improved. After numerous attempts it was found for this case that the $0.1 \mathrm{~Hz}$ sample rate performed well. Cases for this scenario run with the addition of the sun sensor and/or TRIAD performed similarly and required the same sample rate. TRIAD of course provides somewhat shorter convergence time and a lower initial error $\left(9^{\circ}\right.$ versus $37^{\circ}$ for this case $)$. 
Case 7.1.3.2: Perturbed Science, momentum wheel at $1500 \mathrm{rpm}$, magnetometer only, $2 \mathrm{~Hz}$ sample rate

EKF initial state: $x_{0}=[0.005,0.005,0.005,0,0,0,1]^{T}$ (Same as previous)

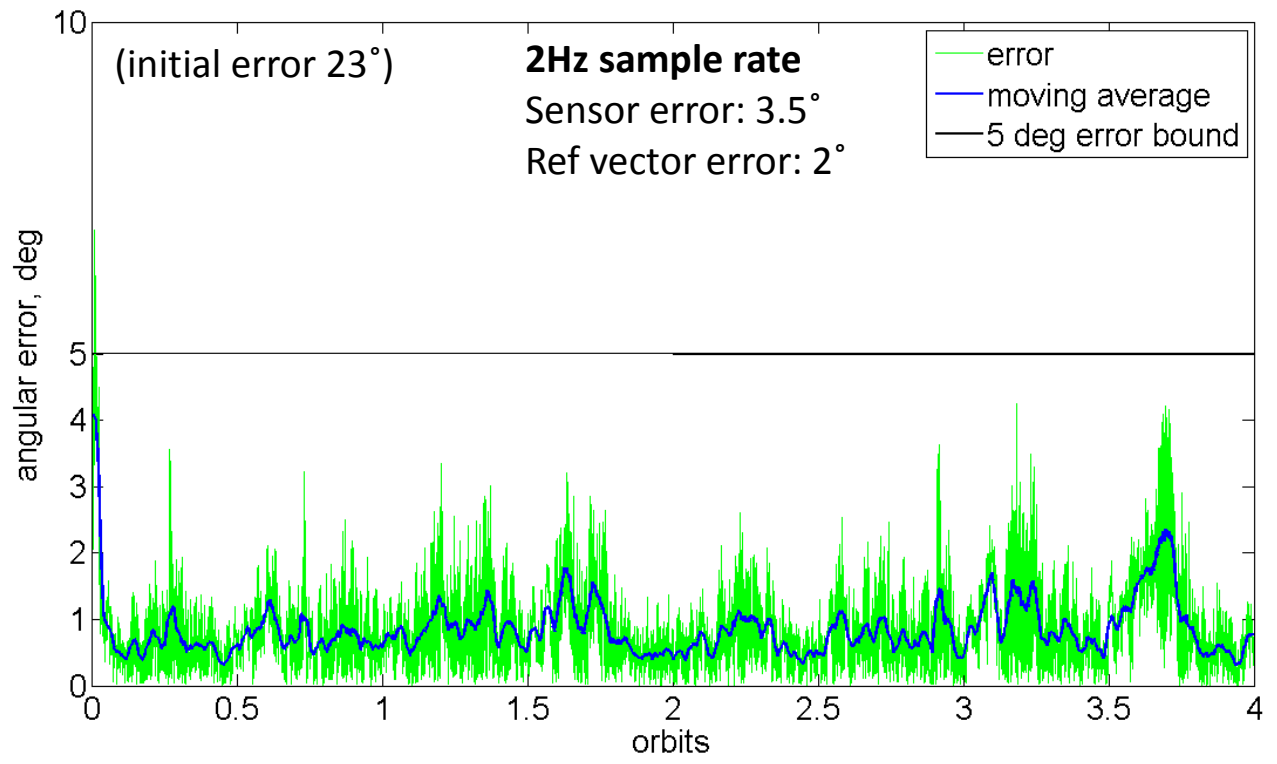

Figure 44. Perturbed Science mode, $1500 \mathrm{rpm}$ wheel rate, magnetometer only at $2 \mathrm{~Hz}$.

A faster sample rate of $2 \mathrm{~Hz}$ is brought back for this case, as the momentum wheelinduced jitter requires it to maintain accuracy. The higher sample rate also results in much faster convergence time compared to the previous case. Passes over the Earth's magnetic poles as described earlier are made very apparent here, and the corresponding rises in error grow slowly grow in magnitude. Although a faster sample rate would lessen this, the steadily increasing periodic rises once again reinforce the need of a future implementation of a means to detect and reset the EKF when steady state error cannot consistently be maintained below the requirement (or any desired level for that matter). 
Cases for this scenario using the sun sensor and TRIAD were conducted but were not crucial to this work, so they will not be presented here for the sake of brevity. Their inclusion however would provide what has been discussed so far: lower steady state error and more rapid convergence.

\subsubsection{MATLAB Simulation Summary}

Pure MATLAB simulations were conducted and analyzed for the crucial "science mode" dynamic state of Exocube, and it was shown that the attitude algorithms developed in this work could feasibly provide knowledge well within the $\pm 5^{\circ}$ mission requirement.

Simulations were then run for a few other dynamic states to explore the potential of implementing the same attitude algorithms for different phases of the Exocube mission aside from the required science mode. A table summarizing all major cases run is shown below.

Table 2. Summary of MATLAB simulation cases.

\begin{tabular}{|c|l|l|l|}
\hline Case & Dynamic State & Sensor(s)/Initial q used & \multicolumn{1}{|c|}{ Notes } \\
\hline 7.1 .1 .1 & Science & mag, assumed aligned & error rises when passing over poles \\
\hline 7.1 .1 .2 & Science & mag, assumed aligned & $\begin{array}{l}\text { error rises over poles more pronounced with } \\
\text { increased wheel speed }\end{array}$ \\
\hline 7.1 .1 .3 & Science & mag, sun, TRIAD & $\begin{array}{l}\text { error rises increasing, need means to detect and } \\
\text { reset EKF }\end{array}$ \\
\hline 7.1 .1 .4 & Science & mag, sun, TRIAD & higher sample rate low error, ideal case thus far \\
\hline 7.1 .2 .1 & Tumble & mag, assumed aligned & $\begin{array}{l}\text { robustness of aligned orientation assumption } \\
\text { discovered }\end{array}$ \\
\hline 7.1 .2 .2 & Tumble & mag, sun, assume aligned & $\begin{array}{l}\text { significantly improved steady state estimation } \\
\text { with addition of sun sensor }\end{array}$ \\
\hline 7.1 .2 .3 & Tumble & mag, sun,TRIAD & "filter smugness" discovered \\
\hline 7.1 .3 .1 & GG Capture & mag, assumed aligned & $\begin{array}{l}\text { much lower sample rate required for calmer } \\
\text { dynamics }\end{array}$ \\
\hline 7.1 .3 .2 & Perturbed & mag, assumed aligned & additional example of need to auto-reset EKF \\
\hline
\end{tabular}




\subsection{MATLAB/STK Simulation}

Because this simulation was constructed primarily as a means of verifying the functionality of the attitude algorithms employed, the simulation parameters were somewhat arbitrary. In the coming example a $1000 \mathrm{~km}, 98^{\circ}$ inclined circular orbit was propagated, and the satellite was set in the science mode state as described in the previous section. The higher orbit was used to avoid having to model drag and prevent any irregularities from arising in the attempted formulation of an "accurate" BSTAR term for the SGP4 TLE. A master step time of 0.5 seconds was set, and the SGP4 propagator used was given only one TLE for initialization. Sensor error applied was the usual $3.5^{\circ}$, while $0.1^{\circ}$ error was added onto reference vectors to account for potential use of magnetic field lookup tables as described by Bowen. ${ }^{1}$ Additional reference vector error generation was not necessary in this simulation since they would already inherit SGP4 orbit propagation error.

Case 7.2.1: Science Mode, momentum wheel at $1500 \mathrm{rpm}$, magnetometer and sun sensor, TRIAD to initialize, $2 \mathrm{~Hz}$ sample rate. STK used for orbit propagation and reference vector generation.

EKF initial state: $x_{0}=\left[0.002,0.002,0.002, q_{1 T}, q_{2 T}, q_{3 T}, q_{4 T}\right]^{T}$. Body rates not matching those in ode 45 but same order of magnitude, TRIAD used to calculate initial quaternion set. 


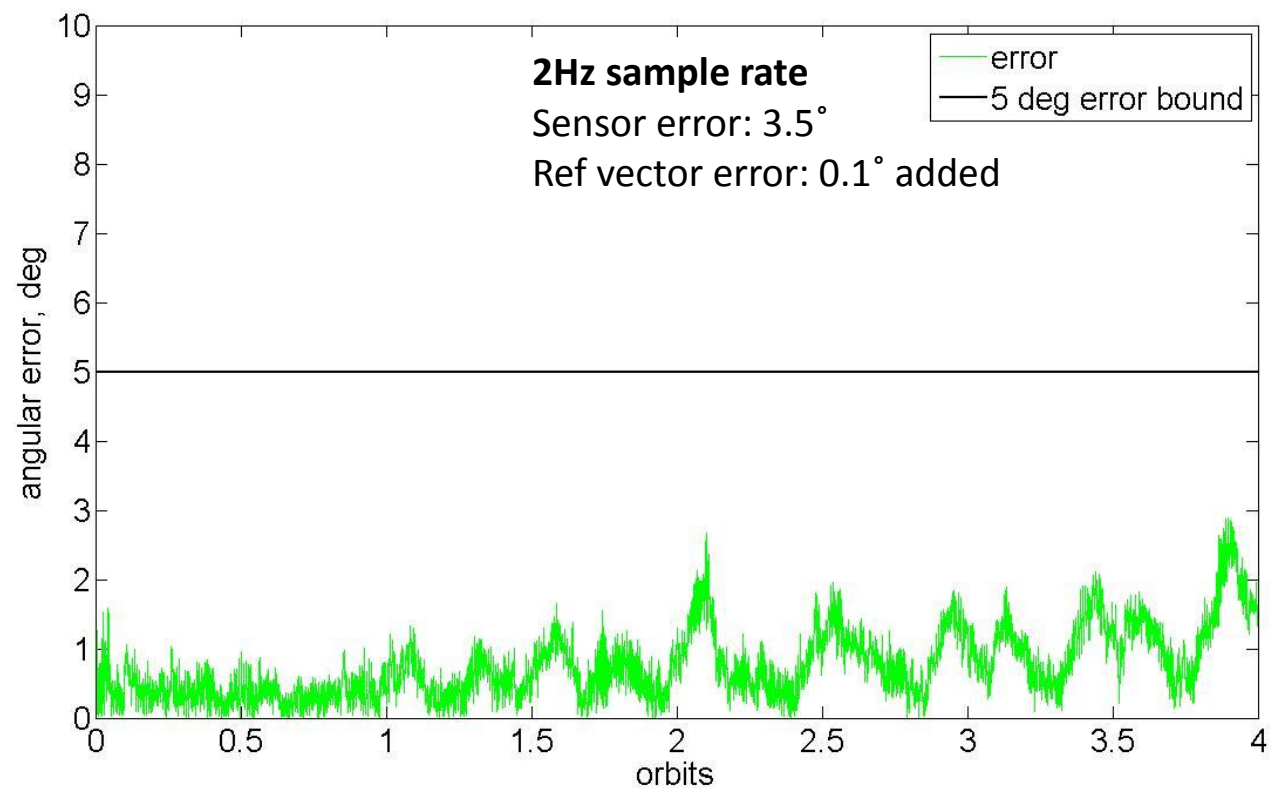

Figure 45. Science mode, STK orbit prop, magnetometer and sun sensor at 2Hz, TRIAD.

Successful convergence was accomplished in this case, verifying that the TRIAD and EKF algorithms researched and developed in this work function properly. However, steady state error appears to grow more rapidly than it should with the propagation of SGP4 error. On investigation, this rather high rate of growth was due to unexpected inconsistencies between the magnetic field values generated by HPOP and those generated by the lookup used for the SGP4 vectors. A plot showing the error between these generated vectors over the course of four orbits (the duration of the simulation) is shown in Figure 46. 


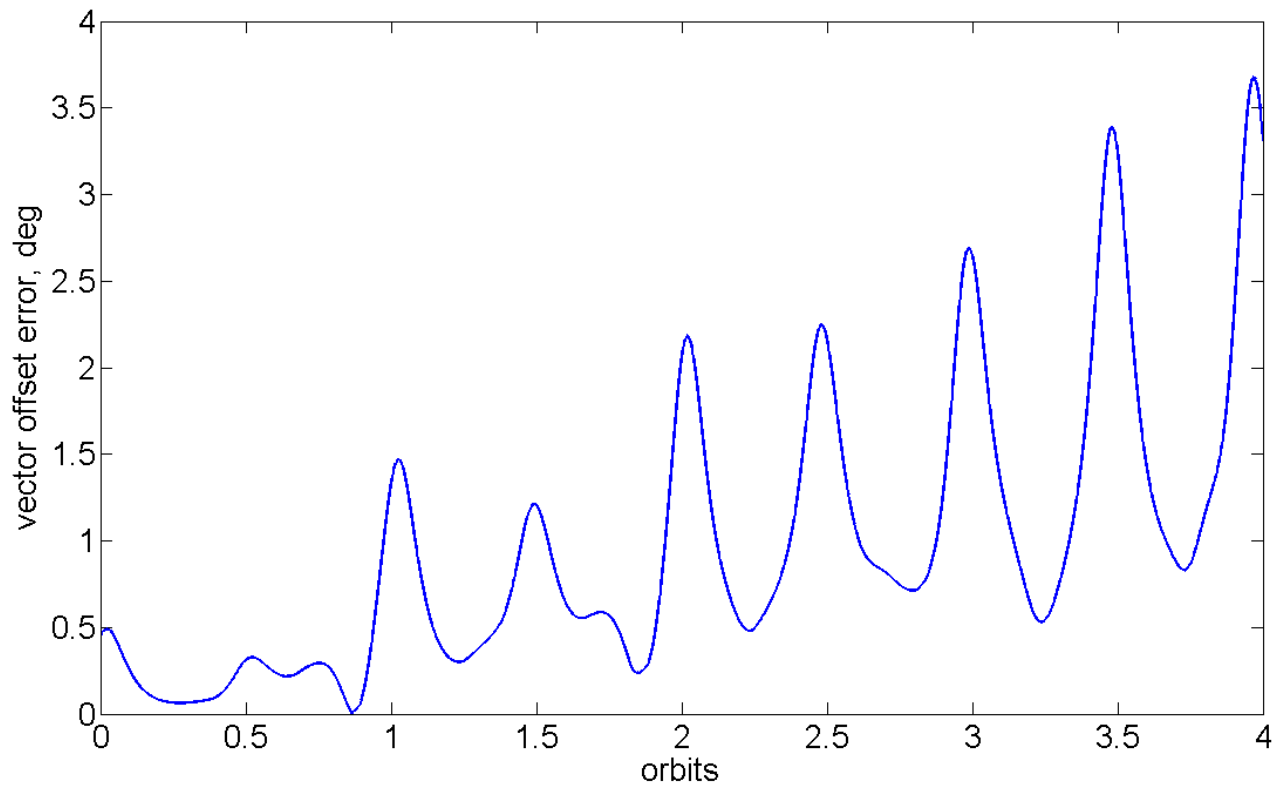

Figure 46. Error between HPOP and SGP4 generated magnetic field vectors.

The error appears somewhat periodic with peaks corresponding to passes over the equator and troughs corresponding to passes over magnetic poles. Bowen's analysis of magnetic field error shows a much lower magnitude of error utilizing the previously mentioned lookup tables. ${ }^{1}$ This would imply that the larger magnitude of error seen here is simply due to different magnetic field models used by HPOP and the magf $f$ function used to generate magnetic field vectors with SGP4 orbit state vectors. Nonetheless, with the proper input parameters confirmed this simulation type can be used in future work to estimate how often the on board SGP4 propagator will need a TLE input in order for the attitude algorithms to maintain a steady state error that falls within the mission requirement for attitude knowledge. 


\subsection{Rate Estimation}

Although not critical to the attitude knowledge mission requirement for Exocube, the body rate estimates output by the EKF are utilized by the on board PD controller commanding magnetorquers. The rate estimates would theoretically be utilized most in the "perturbed science mode" presented, when the spacecraft body rates are at or below the magnitude at which BDOT can provide (see Wertz, Sellers) and the magnetorquers may be required to damp the rates to the level specified by the control requirement $\left(0.1^{\circ} / \mathrm{s}\right) .^{8,11}$ Yaw rate error was consistently highest in the simulations run, and is presented below along with "actual" yaw rate (from ode 45 output) for the perturbed science case (7.1.3.2).
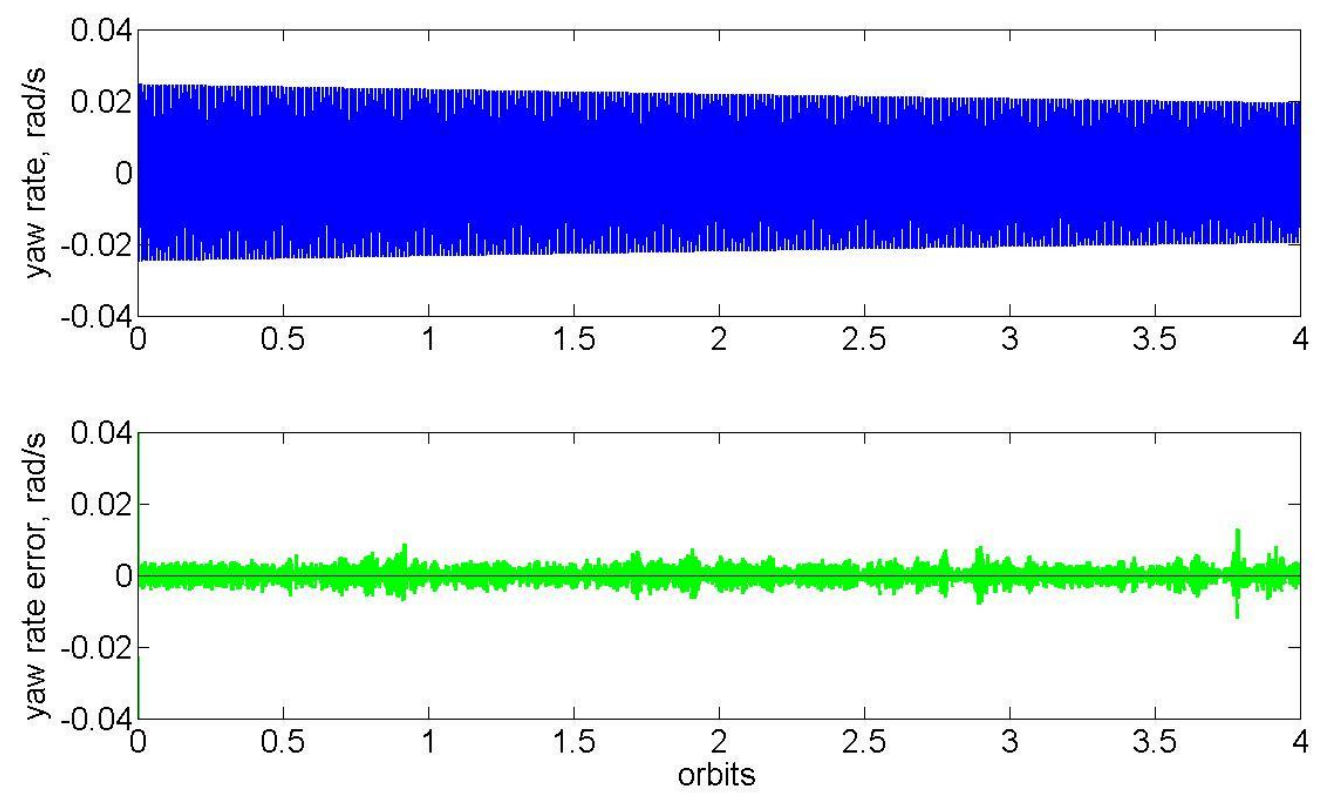

Figure 47. Body yaw rate and associated error for perturbed science case. 
The error shown peaks to greater than $50 \%$ closer to the fourth orbit, but remains much lower otherwise. Despite this high peak, these rate estimates are reported to be working well thus far in control simulations, due to the fact that the rates themselves are still fairly low.

\subsection{Closing Remarks}

The simulations run in this chapter are by no means a conclusive representation of the actual attitude estimation capabilities of Exocube, since the supporting satellite software routines and hardware have not been fully developed at this point. Instead, these simulations serve to prove that the attitude algorithms coupled with the hardware set for use are a feasible means of providing the required $\pm 5^{\circ}$ of attitude knowledge in the "science mode" dynamic state, whose parameters are dictated by the spacecraft control requirements. It was also shown that the estimation routine can successfully be applied to several other anticipated dynamic states throughout the Exocube mission, and very likely to any similar ones.

With the exception of the of the gravity gradient capture mode simulation presented, the EKF used a sample rate of at least $2 \mathrm{~Hz}$. This could be lowered to $0.25-0.20 \mathrm{~Hz}$ for most cases, but with a significant decrease in accuracy; the mission requirement could not be met in science mode with those sampling rates at the level of error set. Algorithm computation times and the respective delay effects on estimation error await further software development for characterization, but the $4 \mathrm{~Hz}$ case run for science mode appears the most ideal for now, providing adequate accuracy with margin without 
requiring an extraordinary amount of computational time or degrading sensor performance.

Ideally, the TRIAD algorithm will always be used in flight to initialize the quaternions fed into the EKF, however several cases were run assuming nominal orientation $(q=$ $\left.\left[\begin{array}{llll}0 & 0 & 0 & 1\end{array}\right]\right)$ to initialize to show the capability of the EKF with regards to convergence in the event that it would need to be started in eclipse or if TRIAD is unavailable for use at the time. Initial inputs for rate estimation have much more leeway, allowing convergence in many cases even if off by one or two orders of magnitude. Due to this flexibility, initial rate estimates are set to within an order of magnitude of that expected in the specific dynamic scenario. Anticipated body rates have yet to be officially characterized for the Exocube mission phases/dynamic states. 


\section{Conclusion}

\subsection{Objective Review}

The aim of this thesis was to research, develop, and prove the feasibility of algorithms that will provide Exocube attitude knowledge precision to within the mission requirement of $\pm 5^{\circ}$ in nominal flight using the basic sensor types selected. The simulations formed would then serve as a tool for this and future PolySat missions in characterizing the attitude estimation capabilities of a given system.

\subsection{Summary}

The algorithms selected and developed for use were TRIAD and an Extended Kalman Filter (EKF). These utilized data from surface mount magnetometers and solar angle sensors modeled with margin added for anticipated errors. Simulations were constructed in MATLAB utilizing an orbit propagator and previously researched ephemeris and lookup models (see Bowen) for generation of reference vectors corresponding to the sensor measurements used. ${ }^{1}$ A "real world" attitude propagation model was coded using ode45 and spacecraft body kinematics/dynamics equations accounting for gravity gradient moments, a pitch axis momentum wheel, an optional spherical damper, effects of an eccentric orbit, and a rough drag model. The EKF modeled equations only for gravity gradient moments, the momentum wheel, and a circular orbit to minimize its complexity.

The EKF was the central focus of this work and was found to perform well enough to not only be used to fulfill the required level of knowledge during "science mode", but also for several other anticipated dynamic states. The TRIAD algorithm was used here as a 
means of "backup" and to aid in initialization of the EKF quaternions, although this is not absolutely necessary in all cases. A final case was run using STK as an external means of orbit propagation and body frame/reference frame vector generation. It not only supplied a means of verifying the attitude algorithms, but resulted in the formation of a simulation that can later be used to help determine how often TLEs need to be uploaded to the on board SGP4 orbit propagator in order to maintain the required level of attitude knowledge throughout mission life.

\subsection{Path Forward}

Currently the EKF and TRIAD algorithms from this work are being integrated into the control scheme developed by Sellers and ported into a Simulink model. ${ }^{8}$ This will provide a means for a full ADCS simulation for Exocube that can take into account finer details such as software process time delays, as well as a foundation for software architecture since all of this will eventually need to be coded in $\mathrm{C}$ and programmed into the Exocube flight boards.

Regarding this work specifically, future steps should involve simulation using actual flight SGP4 code in place of the STK SGP4 code in the second simulation type. Coupled with more precise numbers for sensor errors (once they are calibrated and tested), a much more definitive model of Exocube's attitude estimation capabilities will be known, and can drive decisions on how often TLEs will need to be uploaded and how finely "tuned" the EKF will need to be. More advanced modifications to the EKF may include: 
- Dynamics equations modified for actual body and momentum wheel inertia tensors

- Proper tuning of error covariance and process noise matrix initialization for desired operational modes

- Kinematics/Dynamics model simulating orbit eccentricity, momentum wheel torqueing, magnetic control torques, drag, solar radiation pressure, residual magnetic dipole moment

- Detection of rising steady state error and filter reset method

- Automatically variable filter sampling time to balance computational efficiency against desired accuracy for all portions of orbit

This list can go much further but additions/modifications made to the EKF will only be performed as deemed necessary, as they may of course increase complexity and computation time.

A subject that has not been mentioned earlier in this work is a physical means of attitude verification in flight. The attitude algorithms presented here have been shown to provide a feasible means to meet the mission requirement, but even if/when they do so in flight, they will not directly provide any physical proof. A possible method to account for this can be provided by an on board camera that will be nadir pointing in nominal orientation. Downlinked photos coupled with a consistent timestamp and accurate orbit propagation can compare certain landmarks on Earth with their predicted location relative to Exocube as derived from attitude and orbital position computed. If implemented properly, this can 
result in solid in situ algorithm verification, which would not only confirm successful ADC functionality and good payload data for the Exocube mission, but also help solidify the work done here as a useful simulation tool for future PolySat missions. 


\section{REFERENCES}

${ }^{1}$ Bowen, J. “On-Board Orbit Determination and 3-Axis Attitude Determination for Picosatellite Applications". Aerospace Engineering Master's Thesis, California Polytechnic State University, San Luis Obispo, CA, 2009.

${ }^{2}$ Brown, R. G., and Hwang, P. Y. C. Introduction to Random Signals and Applied Kalman Filtering. $3^{\text {rd }}$ edition. Wiley, New York, 1997.

${ }^{3}$ Curtis, H. D. Orbital Mechanics for Engineering Students. $2^{\text {nd }}$ edition. Elsevier Ltd., 2010.

${ }^{4}$ Hall, C. "Spacecraft Attitude Dynamics and Control”. Lecture notes. Virginia Tech, Blacksburg, VA, 2003.

${ }^{5}$ Humphreys, T. E. “Attitude Determination for Small Satellites with Modest Pointing Constraints”. Monograph, Utah State University, Logan, UT, 2002.

${ }^{6}$ Kane, T. R. Spacecraft Dynamics. Internet-First University Press, Ithaca, NY, 2005.

${ }^{7}$ Modi, V. J., and Shrivastava, S.K. "Satellite Attitude Dynamics and Control in Presence of Environmental Torques - A Survey”. Paper, AIAA, New York, 1982.

${ }^{8}$ Sellers, R. “A Gravity-Gradient, Momentum-Biased Attitude Control System for a Cubesat". Aerospace Engineering Master's Thesis, California Polytechnic State University, San Luis Obispo, CA, 2013.

${ }^{9}$ Sidi, M. J. Spacecraft Dynamics and Control. Cambridge University Press, New York, 1997.

${ }^{10}$ Vallado, D. Fundamentals of Astrodynamics and Applications. $3^{\text {rd }}$ edition. Micrcosm Press, Hawthorne, CA, 2007.

${ }^{11}$ Wertz, J. R., Everett, D. F., and Puschell, J. J. Space Mission Engineering: The New SMAD. Microcosm Press, Hawthorne, CA, 2011.

${ }^{12}$ Wie, B. Space Vehicle Dynamics and Control. AIAA, Reston, VA, 1998.

${ }^{13}$ Zarchan, P., and Musoff, M. Fundamentals of Kalman Filtering. Vol 190. AIAA, Reston, VA, 2000. 